\title{
Strain fields within contracting skeletal muscle
}

Citation for published version (APA):

Maenhout, M. (2002). Strain fields within contracting skeletal muscle. [Doctoral Thesis, Maastricht University]. Maastricht University. https://doi.org/10.26481/dis.20020328mm

Document status and date:

Published: 01/01/2002

DOI:

10.26481/dis.20020328mm

Document Version:

Publisher's PDF, also known as Version of record

\section{Please check the document version of this publication:}

- A submitted manuscript is the version of the article upon submission and before peer-review. There can be important differences between the submitted version and the official published version of record.

People interested in the research are advised to contact the author for the final version of the publication, or visit the DOI to the publisher's website.

- The final author version and the galley proof are versions of the publication after peer review.

- The final published version features the final layout of the paper including the volume, issue and page numbers.

Link to publication

\footnotetext{
General rights rights.

- You may freely distribute the URL identifying the publication in the public portal. please follow below link for the End User Agreement:

www.umlib.nl/taverne-license

Take down policy

If you believe that this document breaches copyright please contact us at:

repository@maastrichtuniversity.nl

providing details and we will investigate your claim.
}

Copyright and moral rights for the publications made accessible in the public portal are retained by the authors and/or other copyright owners and it is a condition of accessing publications that users recognise and abide by the legal requirements associated with these

- Users may download and print one copy of any publication from the public portal for the purpose of private study or research.

- You may not further distribute the material or use it for any profit-making activity or commercial gain

If the publication is distributed under the terms of Article $25 \mathrm{fa}$ of the Dutch Copyright Act, indicated by the "Taverne" license above, 


\section{Strain fields within}

contracting skeletal muscle 
Maenhout, Mascha.

Strain fields within contracting skeletal muscle / by Mascha Maenhout.

- Eindhoven : Technische Universiteit Eindhoven, 2002.

Proefschrift. - ISBN 90-386-2733-5

NUGI 743

Trefwoorden: skeletspieren ; biomechanica / skeletspieren ; rekvelden / spiercontractiemodellen; eindige-elementenmethode

Subject headings: skeletal muscle ; biomechanics / skeletal muscle ; strain fields / muscle contraction models ; finite element method 


\section{Strain fields within contracting skeletal muscle}

\section{PROEFSCHRIFT}

ter verkrijging van de graad van doctor aan de Universiteit Maastricht, op gezag van de Rector Magnificus, Prof.dr. A.C. Nieuwenhuijzen Kruseman, volgens het besluit van het College van Decanen, in het openbaar te verdedigen op donderdag 28 maart 2002 om 14.00 uur

door

\section{Mascha Maenhout}

geboren te Terneuzen op 27 maart 1972 
Promotoren:

Prof.dr. H. Kuipers

Prof.dr.ir. F.PT. Baaijens (Technische Universiteit Eindhoven)

Copromotoren:

Dr.ir. M.R. Drost

Dr.ir. C.W.J. Oomens (Technische Universiteit Eindhoven)

Beoordelingscommissie:

Prof.dr.ir M.G.J. Arts (voorzitter)

Prof.dr.ir. J.L. van Leeuwen (Universiteit Wageningen)

Dr. C.C. van Donkelaar (Technische Universiteit Eindhoven)

Dr. M.K.C. Hesselink

Prof.dr. K. Nicolay (Technische Universiteit Eindhoven) 
Voor mijn vader

Caesar Maenhout 



\section{Contents}

Summary $\quad$ xi

1 Introduction $\quad 1$

1.1 Rationale of the present thesis . . . . . . . . . . . . 1

1.2 Aim and outline ................... 3

2 Anatomy and functioning of skeletal muscle 5

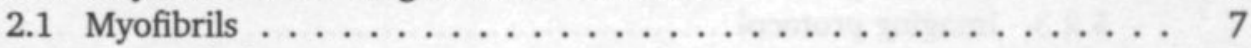

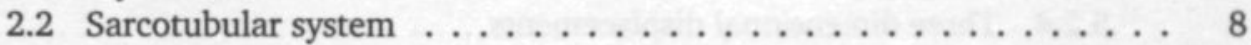

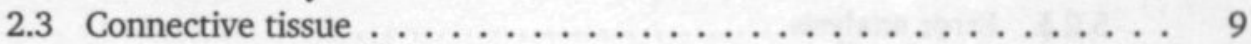

2.4 Molecular mechanisms of contraction . . . . . . . . . . 9

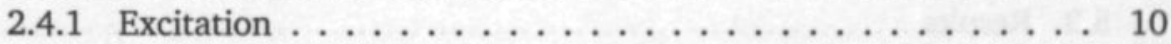

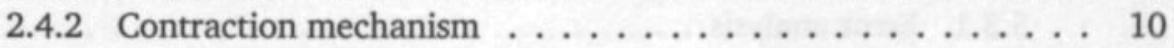

2.5 Functional characteristics .................. 11

3 Parameter identification of a distribution-moment approximated twostate Huxley model

3.1 Introduction . . . . . . . . . . . . . . . . 16

3.2 The model . . . . . . . . . . . . . . . . . . . . 17

3.2.1 The Huxley rate equation with calcium activation . . . . . 17

3.2.2 The distribution-moment (DM) approximation . . . . . . 20

3.2.3 Distribution-Moment model for a muscle-tendon complex . . . 21

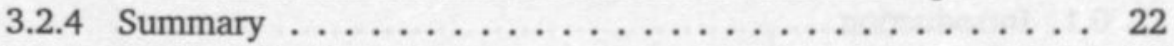

3.3 Parameter identification ... . . . . . . . . . . . 23

3.4 Experiments . . . . . . . . . . . . . . . . . . . 25

3.4 .1 Apparatus description . . . . . . . . . . . 25

3.4 .2 Experimental procedure . . . . . . . . . . 27

3.4 .3 The exercise protocol . . . . . . . . . . . . 27

3.5 Results . . . . . . . . . . . . . . . . . 28

3.6 Discussion . . . . . . . . . . . . . . . . 30

3.7 Future research . . . . . . . . . . . . . . . . 31 
4 Superficial fiber stretch ratios of isometrically contracting muscle based on video analysis

4.1 Introduction . . . . . . . . . . . . . . 34

4.2 Methods . . . . . . . . . . . . . . . . . . . . . 35

4.2.1 Apparatus Description . . . . . . . . . . . 35

4.2 .2 Experimental procedure $\ldots \ldots \ldots \ldots \ldots \ldots \ldots$

4.2 .3 The exercise protocol . . . . . . . . . . . . . . . 37

4.2 .4 Data processing . . . . . . . . . . . . . . 38

4.2 .5 Statistics. . . . . . . . . . . . . . . . . . 39

4.3 Results . . . . . . . . . . . . . . . . . . . . . . . 39

4.4 Discussion . . . . . . . . . . . . . . . . . 43

4.4 .1 General . . . . . . . . . . . . . . . 43

4.4 .2 Experimental procedure . . . . . . . . . . . 43

4.4 .3 Interpretation of results $\ldots \ldots \ldots \ldots \ldots \ldots \ldots$

5 3D strain fields within isometrically contracting muscle based on MR tagged images

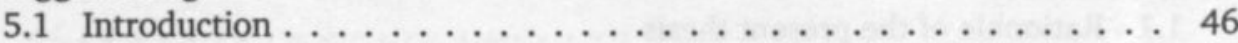

5.2 Methods . . . . . . . . . . . . . . . . . . . 47

5.2 .1 Experimental setup . . . . . . . . . . . . . 47

5.2 .2 Exercise protocol . . . . . . . . . . . . . . 48

5.2 .3 Imaging protocol $\ldots \ldots \ldots \ldots \ldots \ldots \ldots \ldots$

5.2 .4 Three dimensional displacements . . . . . . . . . . 51

5.2 .5 Error analysis . . . . . . . . . . . . . 53

5.2 .6 Statistics . . . . . . . . . . . . . . . . . 54

5.3 Results . . . . . . . . . . . . . . . . . . 55

5.3 .1 Error analysis . . . . . . . . . . . . . 60

5.4 Discussion . . . . . . . . . . . . . . . . 61

5.4 .1 General . . . . . . . . . . . . . . . . 61

5.4 .2 Interpretation of results $\ldots \ldots \ldots \ldots \ldots \ldots \ldots \ldots$

5.4 .3 Error analysis . . . . . . . . . . . . . . 62

5.4 .4 Experimental procedure . . . . . . . . . . . 62

5.4 .5 Future research . . . . . . . . . . . . . . 63

6 Simulated 3D displacement and strain fields within isometrically contracting muscle

6.1 Introduction . . . . . . . . . . . . . . . . 65

6.2 Methods . . . . . . . . . . . . . . . . . . . 66

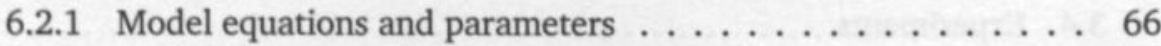

6.2.2 Model geometry of tibialis anterior muscle . . . . . . . . 70

6.2 .3 Simulation settings . . . . . . . . . . . . . 73

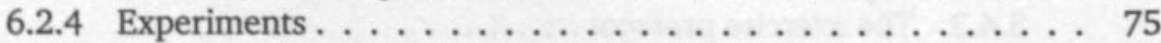

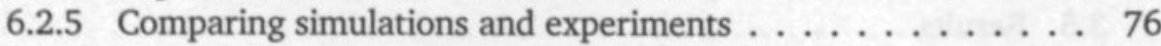

6.3 Results . . . . . . . . . . . . . . . . . 77

6.3 .1 Simulations . . . . . . . . . . . . . . 77 


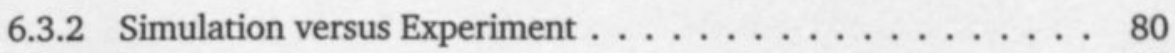

6.4 Discussion . . . . . . . . . . . . . . . . . . . . 85

6.4 .1 General . . . . . . . . . . . . . . 85

6.4 .2 Model parameters . . . . . . . . . . . . 85

6.4 .3 Simulations versus experiments . . . . . . . . . . 87

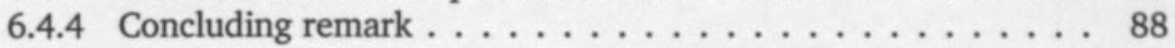

7 Discussion $\quad 89$

7.1 Recapitulation. . . . . . . . . . . . . . . . . . . . 89

7.2 General discussion and recommendations . . . . . . . . . . 91

7.2 .1 Video analysis . . . . . . . . . . . . . . 91

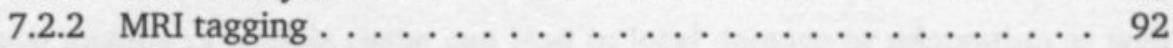

7.2 .3 Model validation . . . . . . . . . . . . . . 92

7.3 Towards damage and adaptation $\ldots \ldots \ldots \ldots \ldots \ldots$

$\begin{array}{lr}\text { References } & 97\end{array}$

$\begin{array}{ll}\text { Samenvatting } & 107\end{array}$

$\begin{array}{ll}\text { Dankwoord } & 111\end{array}$

$\begin{array}{lr}\text { Curriculum Vitae } & 113\end{array}$ 



\section{Summary}

Mechanical loading of muscle tissue can lead to local damage or adaptation as well as to inhomogeneous distributions of stresses and strains within the tissue. These distributions result in locally varying loads which are supposed to be responsible for local changes in properties. Knowledge of the mechanism of local damage and adaptation as a result of local mechanical loading may have important implications for the clinical fields of myoplasty and tendon or muscle transfer. An example of myoplasty is a cardiac assist device, in which transformed skeletal muscle is used to power an assist device. Tendon and muscle transfers can be considered as therapy in restoring the mobility of affected joints by spinal cord injury or in increasing a decreased range of knee motion during gait. Another example of an implication for the clinical field is the development of pressure sores. It is known that muscle tissue is sensitive to prolonged transverse loading and that pressure sores often start in deeper muscle layers near bony prominences. In all the given examples, damage and adaptation are believed to start at a local cell level.

Continuum models with realistic geometries describing the active and passive mechanical behavior of muscle in in vivo situations are powerful tools to study local mechanical loading of muscle tissue in relation to local damage and adaptation. For this purpose, Gielen et al. (2000) developed a sagittal plane model of the tibialis anterior (TA) muscle of the rat.

The aim of the present thesis was 1) to acquire experimental data enabling the validation of continuum models of the rats TA muscle, and 2) to improve the 2D model of Gielen et al. (2000) especially with respect to its geometry. Using the sagittal plane model, various numerical output parameters can be evaluated (e.g. muscle force, displacements, fiber strain, strain gradients). Therefore, an approach was chosen incorporating different measuring techniques to collect an extensive validation data set.

The first experiments, described in chapter 3 , were focused on parameter identification of the contraction model, the so-called distribution-moments (DM) approximated two-state Huxley cross-bridge model. The identification involved fitting 
of a 1D model of the muscle-tendon complex to experimental isometric muscle torque data at different stimulation frequencies. The results indicated that the identified parameters of the DM model enable a good description of the muscle torques of the TA of the rat at different stimulation frequencies. The contraction model is therefor believed to be sufficient for the description of contractile behavior in continuum models of whole muscles during isometric contractions and enables future investigation of metabolic processes. The 1D model however overestimated the experimentally observed rate of torque relaxation, which may be caused by spatial effects. These effects can be investigated in the future with continuum models.

3D superficial marker displacements during electrical stimulation of an isometrically contracting TA muscle of a rat were acquired by $3 \mathrm{D}$ video analysis (chapter 4 ). It was evaluated whether or not the strain distributions depend on 1) the muscle force 2) the joint angle of the rats ankle. Furthermore, it was evaluated how the strain distributions varied during onset and relaxation of the muscle force. The strains during the plateau-phase calculated from the marker displacements were strongly dependent on longitudinal position. The results also indicated that less than $20 \%$ muscle force variation do not have a significant influence on local deformation. Furthermore, variation of the ankle angle of about $30^{\circ}$ caused a strain difference of 0.01 (independent of the longitudinal position). The strain range in logitudinal direction was 0.17 . Therefore, it was concluded that small differences in the angle of the rats ankle (order $5^{\circ}$ ) are not supposed to significantly influence the local deformation. These findings may be important for developing damage inducing exercise protocols, which can be used to find the relation between local damage and local mechanical parameters. Muscle fatigue causing a $20 \%$ decrease of muscle force can be allowed and small differences in the angle of the rats ankle during a damaging protocol do not have a large influence on the strain distributions. Besides the relevance for developing exercise protocols, the experimentally determined relation between local strains and muscle force and muscle length respectively, can serve as validation data for continuum models. During onset and relaxation of the muscle force unexpected phenomena were observed. At the start of the stimulation, while the force increased, proximal regions shortened faster than distal regions. During force relaxation the distal regions showed additional shortening, while the proximal regions lengthened before the initial state was reached. From these phenomena, it was hypothesized that the propagation of the action potential along muscle fibers originating from the motor end-plate strongly influences the time course of the local strain distribution. The hypothesis can be investigated by incorporating inhomogeneous activation of the muscle fibers in a continuum model.

MRI-tagging was employed to acquire the internal 3D tissue deformation during the plateau phase of a fused tetanus (chapter 5). 3D displacement and strain maps of 
about $70 \%$ of the muscle volume were acquired from two sets of orthogonal MR images with tissue tagging of an isometrically contracting TA muscle of a rat. To evaluate the suitability of superficial strains as a measure of deep strains, sagittal strain gradients were determined from the displacement maps. Also transverse strain gradients were determined to evaluate the suitability of sagittal plane models to describe the distribution of mechanical quantities within the whole muscle. The largest differences over a distance of $1 \mathrm{~mm}$ in sagittal and transverse direction of the strain in a particular direction were about $50 \%$ of the maximum absolute strain in that direction. This implies strong heterogeneous strain distributions within the muscle. It was concluded therefore, that 1 ) superficial strains are not a good measure for deeper strains and 2) to study 3D muscle mechanics during contraction, models incorporating realistic $3 \mathrm{D}$ geometries are needed.

The extension of the 2D sagittal plane geometry of (Gielen et al., 2000) of the rat tibialis anterior muscle to a more realistic $3 \mathrm{D}$ geometry is described in chapter 6. The field equations were solved by means of the finite element method, using a mixed, updated Lagrange formulation. A new solid element was employed, which improved the robustness of the computations and the ability to deal with incompressible behavior. The effect of using a $2 \mathrm{D}$ or $3 \mathrm{D}$ geometry on the strain and displacement fields was evaluated as well as the effect of two different sarcomere length distributions (see table 6.3, chapter 6). Furthermore, a step was made towards model validation using the acquired experimental data presented in this thesis. It appeared that the differences between the 2D and 3D simulations were only prominent for the transverse displacements. Furthermore, the initial sarcomere length distribution especially influenced the displacements and strains in longitudinal direction.

With respect to the model validation it became clear that the simulations enabled good qualitative prediction of the MRI displacement fields. However, large quantitative differences were found. Clearly, the model needs further improvement to accurately describe the measured displacement and strain fields. The focus in future studies should be on the following parameters: the 3D geometry and fiber directions, the boundary conditions, the passive constitutive behavior, and the local sarcomere length distributions (see chapter 6, section 6.4.2 for an extensive discussion of these parameters). Although further improvement of the model is needed, the presented 3D model in combination with the extensive experimental data set offers a solid tool to improve the understanding of muscle mechanics during contraction. 


\section{Chapter 1}

\section{Introduction}

\subsection{Rationale of the present thesis}

Skeletal muscle comprises about $40 \%$ of the whole body mass of mammals, and their primary task is to generate force and to move and stabilize parts of the body. Mechanical loading of muscle tissue can lead to local damage or local changes of the tissue properties, referred to as adaptation. These properties include fiber diameter, number of sarcomeres in series in muscle fibers or contractile tension (Koh and Herzog, 1998a; Stauber, 1989; Ebbeling and Clarkson, 1989; Fitts et al., 1991; van der Meulen et al., 1993; Lieber and Fridén, 1993; Lieber et al., 1994). Mechanical loading of the muscle may also lead to inhomogeneous distributions of stresses and strains within the tissue. These distributions result in locally varying loads which are supposed to be responsible for local changes in properties (Ebbeling and Clarkson, 1989; Watson, 1991).

Knowledge of the mechanism of local damage and adaptation as a result of local mechanical loading may have important implications for the clinical fields of myoplasty and tendon or muscle transfer (Brunner, 1995; Koh and Herzog, 1998b). An example of myoplasty is a cardiac assist device, in which transformed skeletal muscle is used to power an assist device (Anderson et al., 1988; Chiu and Bourgeois, 1990; Gealow et al., 1993; Badhwar et al., 1997; Trumble et al., 1997; Mizahara et al., 1999). Tendon and muscle transfers can be considered as therapy in restoring the mobility of affected joints by spinal cord injury or in increasing a decreased range of knee motion during gait (Bliss and Menelaus, 1986; Illert et al., 1986; Delp et al., 1994; Keith et al., 1996). Another example of an implication for the clinical field is the development of pressure sores. It is known that muscle tissue is sensitive to prolonged transverse loading and that pressure sores often start in deeper muscle layers near bony prominences. In the given cases, damage and adaptation are believed to start at 
a local cell level (Bosboom et al., 2001a).

Models to study the mechanics of contracting skeletal muscle have been reported in literature. Huxley (Huxley, 1957) and Hill (Hill, 1938) type contraction models in series with a spring representing the tendon (figure 1.1a) are generally used in gait analysis and muscle performance studies (Hatze, 1981; Winters and Woo, 1990).

(a)

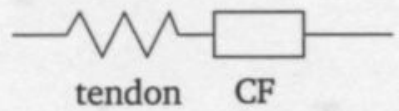

Figure 1.1: Models of muscle-tendon complexes: (a) model with a spring to represent the tendon and contractile fibers (CF). (b) parallelogram model of a unipennate muscle, with straight contractile fibers (CF) and rigid tendons and aponeuroses. The areas indicated by the asterisks are mechanically unstable, as can be identified by looking at the conservation of momentum and conservation of moment of momentum.

To incorporate some of the geometrical aspects of muscles, straight line pennate muscle models (e.g. parallelogram models, figure 1.1b) were designed (Woittiez et al., 1984; Kaufman et al., 1989). The stress and strain distributions were considered homogeneous and the purpose of these models was to study force-length and force-velocity relationships of whole muscle. A drawback of these models is that they are in conflict with the laws of mechanical equilibrium as was explained by van Leeuwen and Spoor (1992) due to the straight-line geometry. Van Leeuwen (1996) composed a mechanically stable 2D fiber-fluid model to study the pressure in muscles during contraction. The curved unipennate geometry was based on the functional demand of equal shortening of muscle fibers. The contribution of the connective tissue of the muscle was neglected. A finite element skeletal muscle model with a 2D curved unipennate geometry was presented by van der Linden et al. (1998a). The model consisted of parallel muscle elements, incorporating passive and active behavior, and connective tissue elements representing the tendons and aponeuroses. The mentioned models are useful tools in studies towards muscle mechanics. However, due to the assumption of homogeneous behavior in the fiber direction, they are less suitable to study local mechanical loading, which is believed to result in local damage or adaptation. For this purpose, continuum models with realistic geometries describing the active and passive mechanical behavior in in vivo situations are needed. Gielen et al. (2000) developed a sagittal plane model of the tibialis anterior (TA) of the rat to study the stress and strain distributions within contracting skeletal muscle. The contractile behavior was described with a two-state Huxley cross-bridge model 
(Zahalak, 1981; Zahalak and Ma, 1990). The equations were solved numerically with the finite element method, resulting in a solution for the material deformation, strain and stress.

\subsection{Aim and outline}

To study heterogeneous aspects of skeletal muscle contraction, continuum models like the model of Gielen et al. (2000) can be used. The purpose of the present thesis was 1) to acquire experimental data enabling the validation of continuum models of the rats TA muscle, and 2) to improve the 2D model of Gielen et al. (2000) especially with respect to its geometry. Using the sagittal plane model, various numerical output parameters can be evaluated (e.g. muscle force, displacements, fiber strain, strain gradients). Therefore, an approach is chosen incorporating different measuring techniques to collect an extensive validation data set.

This thesis is written such that all chapters can be read separately from each other. Skeletal muscle anatomy and functioning will be briefly introduced in chapter 2 .

The first experiments, which are described in chapter 3 , were focused on parameter identification of the contraction model of the muscle. This model is based on a twostate Huxley cross-bridge theory, including the calcium activation dynamics according to Zahalak and Ma (1990), which was an improvement compared to the model of Gielen (1998). The identification involved fitting experimental isometric muscle torque data at different stimulation frequencies to a 1D model of the muscle-tendon complex by minimizing a least squares objective function. Chapter 3 is published as Maenhout et al. (2000), and in that form it is included in this thesis.

Chapter 4 describes deformation measurements at the muscle surface. 3D superficial marker displacements during electrical stimulation of an isometrically contracting TA muscle of a rat were acquired by $3 \mathrm{D}$ video analysis. It was evaluated whether the strains depend on 1) the muscle force 2) the joint angle of the rats ankle. Furthermore, it was evaluated how the strain distributions varied during onset and relaxation of the muscle force. Chapter 4 will be submitted for publication as Maenhout et al. (2001b).

A MRI-tagging technique, described in chapter 5, was employed to acquire the internal 3D tissue deformation during the plateau phase of a fused tetanus. Displacement maps were acquired from two sets of orthogonal MR images with tissue tagging of an isometrically contracting TA muscle of a rat. To evaluate the suitability of superficial strains as a measure of deep strains, sagittal strain gradients were determined from the displacement maps. Also transverse strain gradients were determined to evaluate the suitability of sagittal plane models for the simulation 
of the distribution of mechanical quantities (like stain) within the whole nuscle. Chapter 5 is submitted for publication as Maenhout et al. (2001a), and in that form it is included in this thesis.

Chapter 6 describes the extension of the sagittal plane continuum model to ${ }_{a}$ more realistic $3 \mathrm{D}$ geometry. Moreover, the contraction model was implemented in a new solid element, especially suitable for studies on incompressible materials. At the end of chapter 6 a comparison of the simulations with MRI and video data will be described. This chapter was chosen not to be written in the form of a publicati, , but can be read separately from the other chapters.

Finally, the implications of the research presented in this thesis and recommenlations for future research are discussed in chapter 7 . 


\section{Chapter 2}

\section{Anatomy and functioning of skeletal muscle}

Muscle fibers and a connective tissue network are the main components of the muscle tissue. At different levels in the muscle tissue sheets of connective tissue are observed, holding the muscle components together (figure 2.1) and enable force transmission. The muscle is surrounded by a thick outer connective tissue, called the epimysium. The perimysium divides the muscle further into fascicles, which are bundles of muscle fibers. Each fascicle contains a few to over a hundred muscle fibers, which are surrounded by a thinner connective tissue sheet, the endomysium.

The muscle fibers span the length of the entire muscle or only a part of it. They always end in tendons or other connective tissue intersections. The length of the fibers ranges from a few millimeters to several centimeters, while their diameter is in the range of $10 \mu \mathrm{m}$ to $100 \mu \mathrm{m}$.

The skeletal muscle fiber differs from other cell types by the presence of multiple nuclei, because it is formed by fusion of many myoblasts in the embryonal phase. The cytoplasm contains myofibrils (figure 2.1) that convert metabolic energy into mechanical energy, and a sarcotubular system needed for the initiation of contraction by the release of $\mathrm{Ca}^{2+}$ into the muscle cell. A detailed description of the myofibrils and the sarcotubular system is given in the following two subsections. 


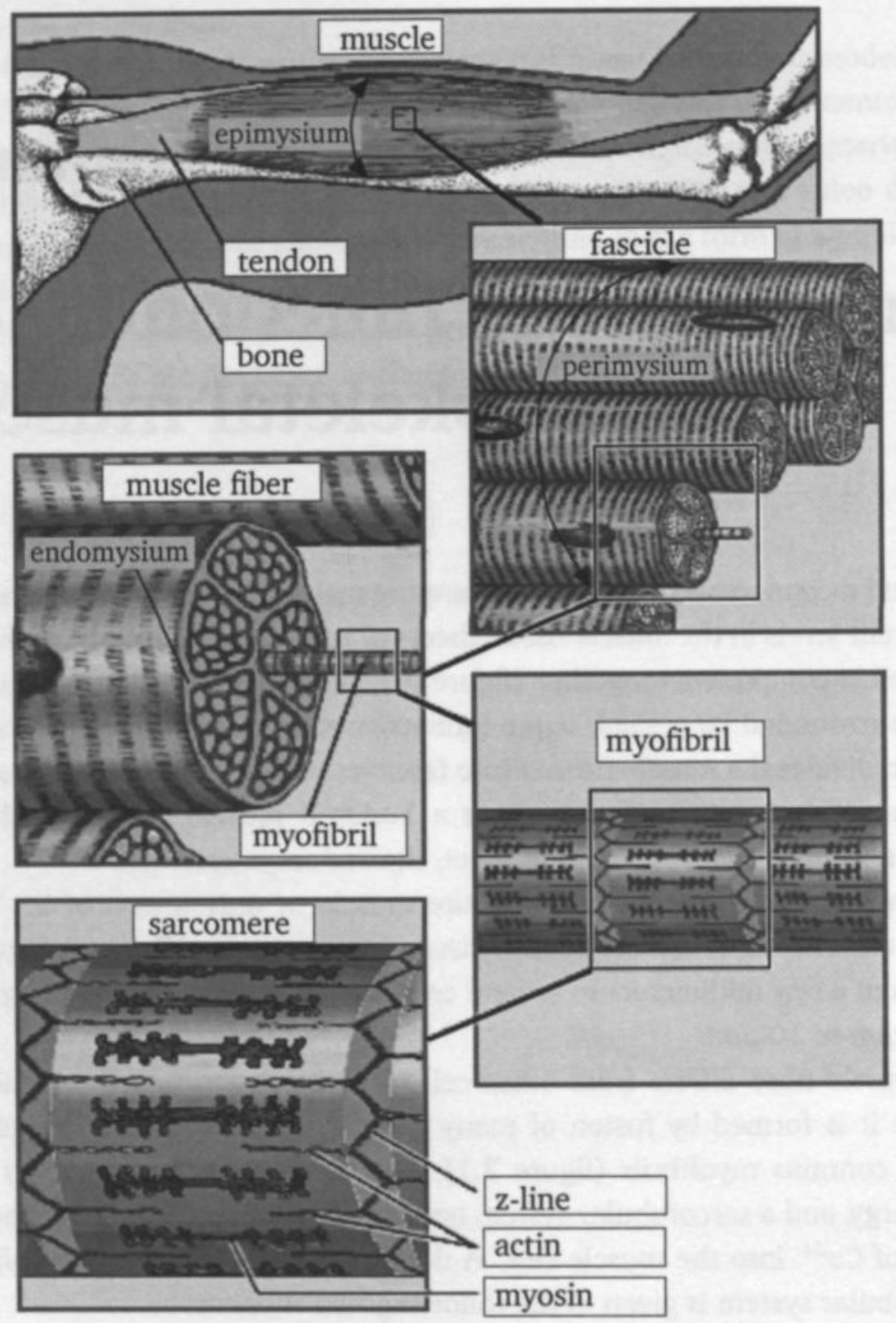

Figure 2.1: Muscle gross structure. The entire muscle is covered with epimysium which continues as the perimysium covering the bundles of muscle fibers named the fascicles. Finally it ends as the endomysium covering the muscle fibers. Adapted from Encyclopaedia Britannica, 1994. 


\subsection{Myofibrils}

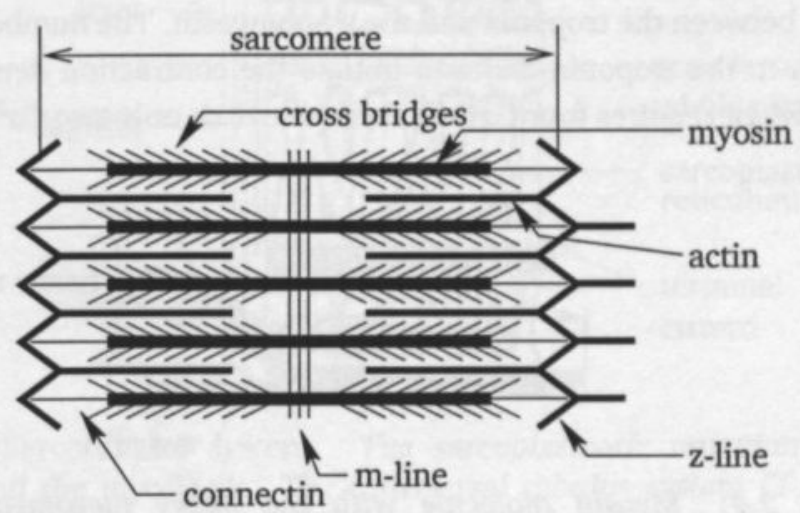

Figure 2.2: The sarcomere: The myosin filaments with the cross-bridges interdigitate with the actin filaments. The connectin, also called the titin molecule, connects the myosin to the $\mathrm{z}$-line.

The myofibrils are composed of repeating units of actin and myosin filaments, called sarcomeres (see figure 2.2). The actin filaments are attached at one end to the $\mathrm{Z}$ line, also called $\mathrm{z}$-disk, and are free at the other end to interdigitate with the myosin filaments. When an actin and myosin filament attach, a so-called crossbridge is formed. The myosin filaments are connected to the z-disk by means of a connecting molecule titin (Skubiszak, 1993), also called connectin, that contributes to the stiffness of the muscle fiber and protects the sarcomere against over-stretching (Keller, 1997; Tskhovrebova et al., 1997).

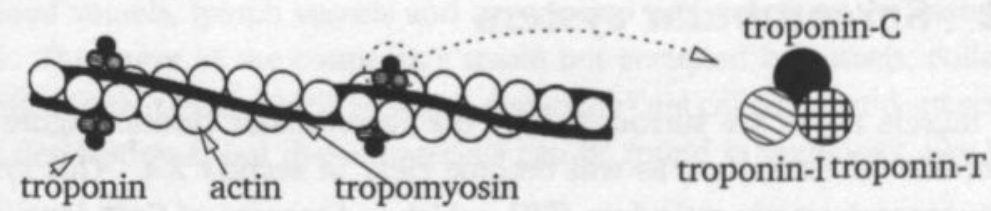

Figure 2.3: The actin filament is a double helix of actin with troponin at every half-turn and tropomyosin as regulatory proteins. The troponin consists of three subunits, that connect $\mathrm{Ca}^{2+}(\mathrm{C})$, inhibit contraction (I), and connect to the tropomyosin (T).

The actin filament is composed of a double stranded helix of actin molecules with troponin molecules in the strand at every half-turn (see figure 2.3). The troponin molecules are interconnected by tropomyosin molecules. The troponin is made of 3 
subunits. The troponin-I inhibits the interaction of myosin with actin and troponin$\mathrm{C}$ contains sites for $\mathrm{Ca}^{2+}$-ions that initiate contraction. The troponin- $\mathrm{T}$ forms the connection between the troponin and the tropomyosin. The number of $\mathrm{Ca}^{2+}$-ions that must attach to the troponin- $\mathrm{C}$ site to initiate the contraction determines the twitch type: fast-twitch requires four $\mathrm{Ca}^{2+}$-ions, slow twitch only two $\mathrm{Ca}^{2+}$-ions.

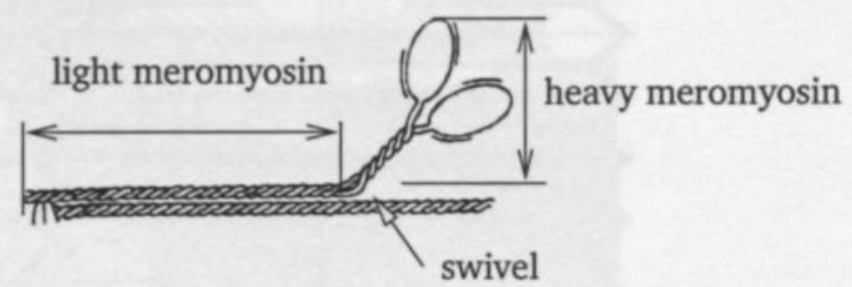

Figure 2.4: Myosin molecule with the heavy meromyosin and light meromyosin.

The myosin filament is composed of myosin proteins with a complex structure. The form resembles a 'golf-club': the sticks of the club merge in the myosin filament. This part of the molecule is called the light meromyosin. The head of the stick is the heavy meromyosin, that is composed of two myosin heads at the end and a connecting strand, also called swivel, between the heads and the light meromyosin (figure 2.4). The heads and the swivel play an important role in the contraction process.

\subsection{Sarcotubular system}

The muscle fibrils are surrounded by the sarcotubular system (figure 2.5), that is important for excitation, as will become clear in section 2.4. This system consists of the sarcoplasmatic reticulum (SR), which is a storage of $\mathrm{Ca}^{2+}$-ions. Furthermore, the system is made up of a transversal tubulus system (T-system), that conducts the depolarisation wave in the outer membrane to the site where $\mathrm{Ca}^{2+}$-ions are released. The SR forms an irregular curtain around each of the fibrils. It spans one or several T-systems. The contact of the SR with the T-system is also referred to as the terminal cisterns. The T-system rapidly transmits the action potential from the cell membrane to all SR's surrounding the fibrils. The contact of the T-system with the SR is closed, but transmitter molecules can flow from the T-system to the SR. The transmitter molecules initiate an action potential on the SR. 


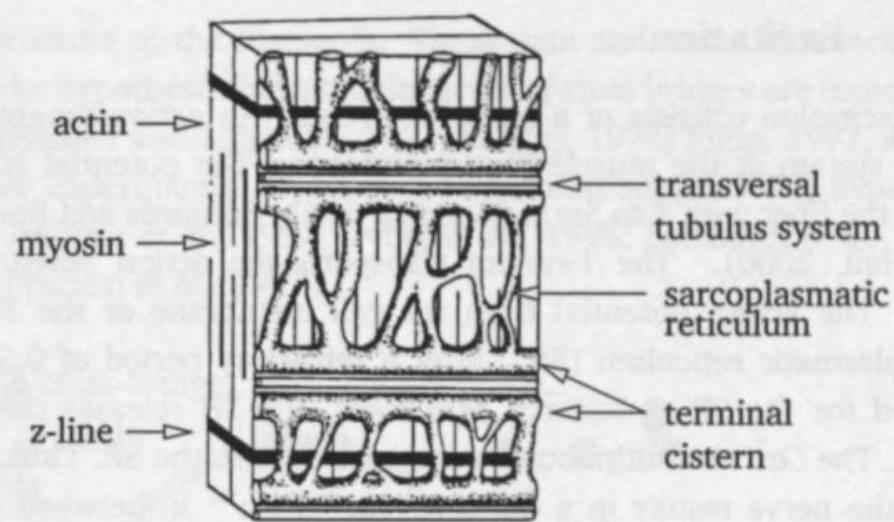

Figure 2.5: Sarcotubular system. The sarcoplasmatic reticulum forms curtains around the myofibrils. The transversal tubulus system (T-system) is at the same location along the fiber as the ends of the myofibrils, resulting in two T-systems per sarcomere.

\subsection{Connective tissue}

The connective tissue transmits the force through and along the muscle fibers onto the skeleton. The ends of the muscle fiber are tightly connected to connective tissue at the myo-tendinous junctions (Tidball, 1991). Furthermore, the endomysium transmits the force along the muscle fibers to the perimysium, that in its turn transmits it through the epimysium to the tendons. The perimysium and endomysium allow individual fibers and bundles to move independently within a certain range. The epimysium forms the outer layer, that allows relative movement of the muscles. The connective tissue consists of collagen and elastin fibers and also harbors the systems of blood vessels, lymph vessels and nerves into the muscle to the individual muscle fibers. The space in the connective tissue not occupied by vessels, collagen, or elastin is filled with a hydrophilic (=water attracting) gel called ground substance. An extensive description about these materials can be found in textbooks, like Fung (1993).

\subsection{Molecular mechanisms of contraction}

When a muscle is activated by the nerve system there are two major processes: the excitation and the so called cross bridge process. Excitation is an electrical phenomenon, that is responsible for the transport of $\mathrm{Ca}^{2+}$, into the muscle fiber. The cross bridge process describes the contraction mechanism, involving the attachment and detachment of myosin filaments to actin filaments. 


\subsubsection{Excitation}

The excitation consists of a series of events. An action potential arrives from the nerve system at the muscle fiber membrane. The potential is rapidly transported along the fiber with 1 to $5 \mathrm{~m} / \mathrm{s}$ (Ganong, 1981; Bernards and Bouman, 1988; Guyton and Hall, 2000). The T-system transports the action potential into the muscle fiber. The action potential from the cell membrane or the T-system triggers the sarcoplasmatic reticulum (SR). After a refractory period of 0.5 to $3.0 \mathrm{~ms}$, the time needed for the SR to become permeable, the SR releases the $\mathrm{Ca}^{2+}$ ions into the fibrils. The $\mathrm{Ca}^{2+}$ is continuously pumped back into the SR. Thus, the action potential from the nerve results in a quick release of $\mathrm{Ca}^{2+}$ in between the fibrils. A single action potential (pulse) causes a brief rise in the interfibrilar $\mathrm{Ca}^{2+}$ concentration, immediately followed by a fall in concentration. Such an action is called twitch. The duration of the twitch depends on the fiber type. A longer lasting high $\mathrm{Ca}^{2+}$ concentration is obtained by sending more impulses to the SR (figure 2.6). At the so called critical fusion frequency, the $\mathrm{Ca}^{2+}$ concentration does not show a ripple anymore. This state is called tetanized state. The frequency of the stimulation pulses controls the average $\mathrm{Ca}^{2+}$ concentration.
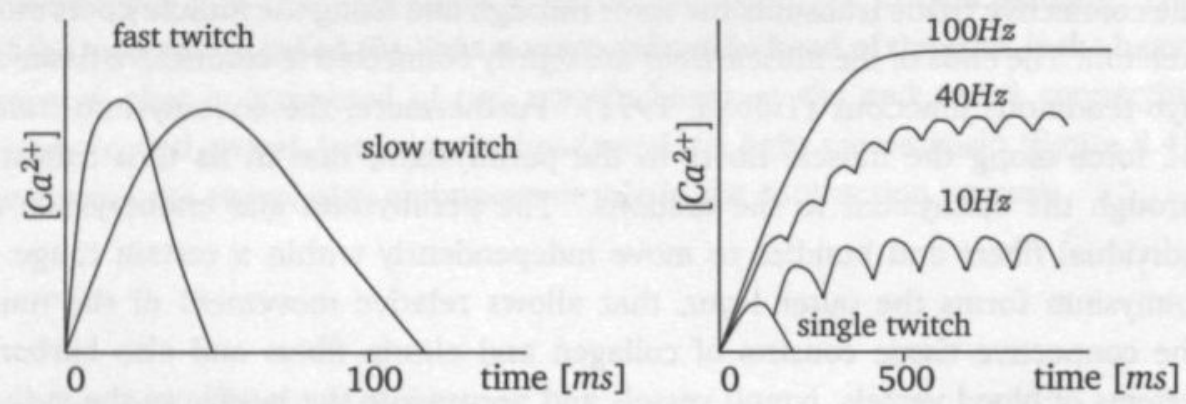

Figure 2.6: Left: single twitch for different fiber types. Right: wave summation. The labels indicate the stimulation frequency.

\subsubsection{Contraction mechanism}

The basis of the contraction mechanism was firstly described by the sliding filament theory. This theory was derived from observations of the sarcomeres in the muscle fibers independently by Huxley and Niedergerke (1954) and Huxley and Hanson (1954). Their studies revealed that the actin and myosin filaments do not change length during a contraction. Therefore, a mechanism must exist to move the filaments mutually. Electron microscopy and X-ray diffraction studies of actin and myosin 
showed a regular structure of the filaments. These data and many bio-mechanical experiments lead to the hypothesis that a regular array of cross bridges are responsible for the contraction (Huxley and Simmons, 1971; Huxley, 1974; Fung, 1993, section 9.10). Recently, more experimental evidence is found that supports this hypothesis in great detail (e.g. Ishijima et al., 1991; Irving et al., 1995; Lombardi et al., 1995; Molloy et al., 1995; Piazzesi et al., 1997).

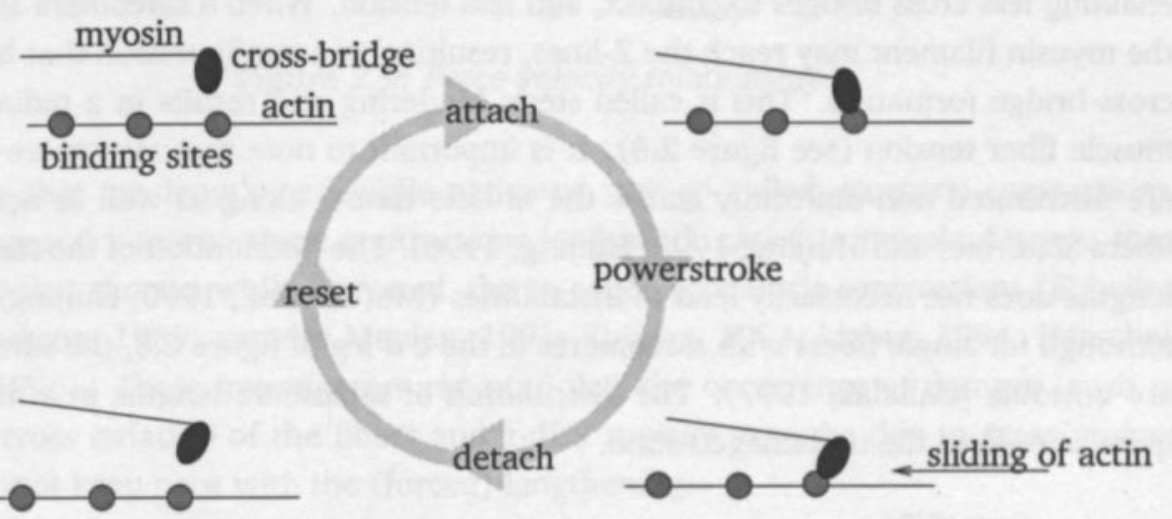

Figure 2.7: Sliding filament model: When the troponin molecules are activated by the $\mathrm{Ca}^{2+}$ ions, then the myosin molecules can attach to the actin molecules. The myosin molecules swivel, producing a power stroke resulting in a sliding of the actin filaments. The myosin molecule detaches from the actin and is reset for the next cycle.

The $\mathrm{Ca}^{2+}$, released from the SR, binds with the troponin- $\mathrm{C}$. This results in a change of spatial configuration of the troponin molecule, such that the tropomyosin uncovers the attachment sites of the actin molecule. The heads of the myosin molecules link to actin at an angle, producing movement of myosin on actin by swiveling (rotating the heads). The heads disconnect and reconnect at a linking site, repeating the process in a serial fashion (figure 2.7). Each cycle of attaching, swiveling and detaching shortens the sarcomere by $1 \%$. When the $\mathrm{Ca}^{2+}$ concentration in the myofibrilar space gets low, the troponin- $\mathrm{C}$ sites release the $\mathrm{Ca}^{2+}$ ions. The tropomyosin covers the attachment sites again, disabling the attachment of the myosin molecules.

\subsection{Functional characteristics}

There are three main types of muscle fibers. The types are called type I (slow twitch, red, oxidative, fatigue resistant), type IIa (fast twitch, white, oxidative and glycolytic, fatigue resistant), and type IIb (fast twitch, white, glycolytic, not fatigue resistant). 
Depending on the function of the muscle there is a preponderance of one of the fiber types (Armstrong and Phelps, 1984; de Ruiter, 1996).

The cross bridge cycle described in the previous paragraph causes tension in the muscle fibers. This contractile tension depends, among others, on the length of overlap of the actin and myosin filaments in the sarcomere (i.e., the number of cross bridges). An extension of the sarcomere results in a reduction of overlap area, enabling less cross bridges to connect, and less tension. When a sarcomere shortens, the myosin filament may reach the Z-lines, resulting in a configuration that hampers cross bridge formation. This is called steric hindering and results in a reduction of muscle fiber tension (see figure 2.8). It is important to note that sarcomere lengths are distributed non-uniformly across the muscle tissue, along as well as across the fibers (Zuurbier and Huijing, 1993; Huijing, 1996). The distribution of the sarcomere lengths does not necessarily lead to instabilities (Moran et al., 1990; Huijing, 1996), although for single fibers with sarcomeres in the c-d leg of figure 2.8, the sarcomeres are unstable (Zahalak, 1997). The distribution of sarcomere lengths in a muscle is quite smooth in the undamaged state.

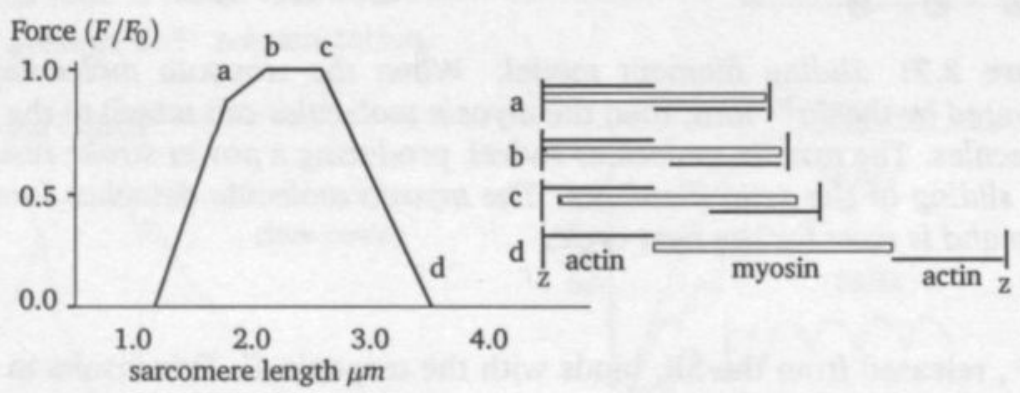

Figure 2.8: Length dependence of contraction. The relative sarcomere tension $F / F_{0}$ against the sarcomere length (left). The points $a, b, c$, and $d$ correspond to a certain sarcomere configuration (right).

The contractile tension or muscle force is also related to the contraction velocity. The cross bridges drive the contraction when the shortening velocity is low. At high shortening velocities the cross bridges can just keep pace, producing little tension, or even tension opposing the movement. This behavior can be represented by a hyperbolic shaped force-velocity relationship, as observed by Hill (see figure 2.9). 


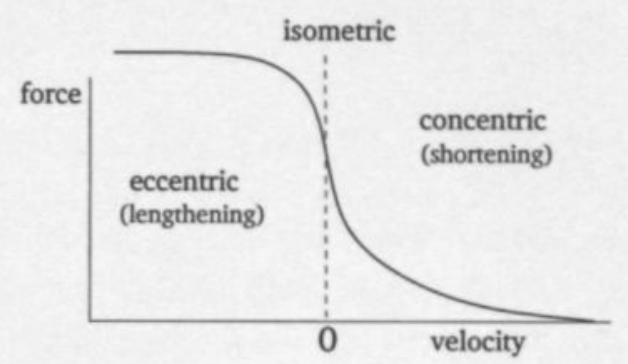

Figure 2.9: Force-velocity relationship.

Muscles that are lengthened while activated, the so called eccentric contractions, are of special interest: these contractions lead much easier to muscle damage than muscles that shorten while activated, the so called concentric contractions (Ebbeling and Clarkson, 1989; van der Meulen, 1991; Kuipers, 1994; Lieber, 1994; Hesselink et al., 1996). These investigators reported that the occurrence of damage, such as loss of cross striation of the fibers and z-disk rupture, may be due to cross bridges that cannot keep pace with the (forced) lengthening. 



\section{Chapter 3}

\section{Parameter identification of a}

distribution-moment

\section{approximated two-state Huxley model}

M. Maenhout, M.K.C. Hesselink, C.W.J. Oomens, M.R. Drost

Parameter identification of a Distribution-Moment approximated two-state Huxley model of the rat tibialis anterior muscle, Skeletal Muscle Mechanics; From Mechanism to Function editor: Walter Herzog, publisher: John Wiley \& Sons, chapter 9, 2000, pages: 135-154, ISBN 0-471-49238-8. 


\subsection{Introduction}

Mechanical loading of muscle tissue can lead to regional changes in the tissue such as fiber diameter, number of sarcomeres in series in muscle fibers, or even regional damage (Ebbeling and Clarkson, 1989; van der Meulen et al., 1993; Lieber et al., 1994; Koh and Herzog, 1998a). External loads of the muscle (e.g. active muscle force) results in regional loads (stresses and strains) within the tissue, which are supposed to be responsible for the regional changes (Ebbeling and Clarkson, 1989; Watson, 1991). The process of changes is referred to as adaptation. Knowledge of the mechanisms of damage and adaptation may have important implications for the clinical fields of myoplasty and tendon or muscle transfer (Brunner, 1995; Koh and Herzog, 1998b). An example of myoplasty is a cardiac assist device, in which transformed skeletal muscles is used to power an assist device (Gealow et al., 1993; Badhwar et al., 1997; Trumble et al., 1997; Mizahara et al., 1999). Tendon and muscle transfers can be considered as therapy in restoring the mobility of joints affected by spinal cord injury or in increasing a decreased range of knee motion during gait (Bliss and Menelaus, 1986; Illert et al., 1986; Delp et al., 1994; Keith et al., 1996).

To study the influence of mechanical load on muscle tissue a continuum model of the tibialis anterior (TA) of the rat is developed by Gielen (1998). The geometry of the continuum model is based on a MRI image of a cross-section of the hind-limb of a rat. The local fiber direction is determined by diffusion-weighted MRI. A distributed moments (DM) approximated two-state Huxley model (Zahalak, 1981; Ma, 1988; Zahalak and Ma, 1990; Zahalak and Motabarzadeh, 1997) is used to describe the contractile properties. The passive tissue is described by a 3D non-linear anisotropic elastic model. Although 3D hexahedral elements are used the continuum model represents a 'flat slice' in the median plane of the TA. The equations covering the continuum model are solved with the finite element method.

In order to determine whether or not the model describes the real stresses and strains within the muscle tissue, unknown parameters need to be identified, and the model needs to be validated. This paper will discuss the first validation step that was focused on the contraction model or more specific, the distribution-moments approximated two-state Huxley cross-bridge model including calcium activation dynamics (Zahalak, 1981; Ma, 1988; Zahalak and Ma, 1990; Zahalak and Motabarzadeh, 1997). A big advantage of this model compared to the Hill-type models is the integration of mechanical, structural, and biochemical features of muscle tissue in one model. The parameters can be interpreted physically and the model is suitable for an inclusion of metabolic processes. The objective of the present research is to identify the unknown DM parameter values for the rat tibialis anterior (TA) muscle. 


\subsection{The model}

Because model simulations will be compared to macroscopic behavior like muscle torque, a 1D model of the muscle-tendon complex was used to identify the unknown parameters. This 1D model consists of a force generating contractile element described by the DM-approximated two-state Huxley model, in series with a nonlinear spring representing the tendon (figure 3.1).

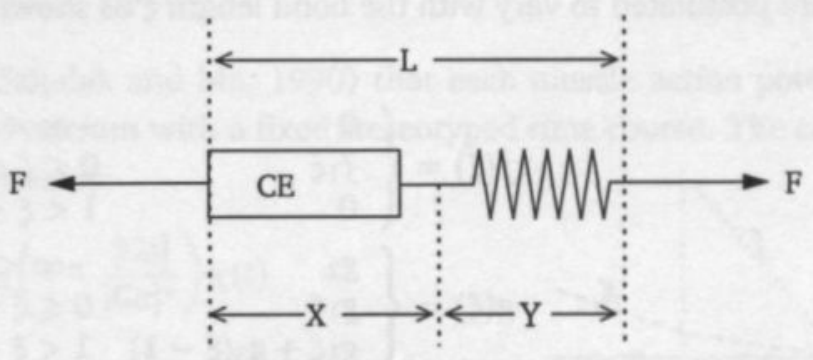

Figure 3.1: The 1D muscle model consisting of a contractile element (CE) generating force and an elastic spring element. $L$ represents the length of the whole muscle tendon complex, $X$ is the length of the contractile tissue and $Y$ represents the length of the tendon.

A parallel elastic element representing the passive behavior of the muscle tissue was omitted, because for an actively contracting muscle in the physiological range of the muscle lengths, the passive force is negligible compared to the active force (Hill, 1938, 1950).

\subsubsection{The Huxley rate equation with calcium activation}

The contractile property of the muscle tissue is described by a two-state Huxley crossbridge model. The basic Huxley theory focuses on an ensemble of myosin heads which are assumed to be capable of binding to an actin binding site to form a so called cross-bridge. Since the original equation was postulated by Huxley (1957), many modifications were applied in order to improve the agreement of the model with experimental results. To include the calcium activation and the filament overlap, $\mathrm{Ma}$ and Zahalak modified the original equation to the so-called 'generalized Huxley equation including calcium activation' (Ma, 1988; Zahalak and Ma, 1990; Zahalak and Motabarzadeh, 1997). The equation reads:

$$
\frac{d n(\xi, t)}{d t}=\frac{\partial n(\xi, t)}{\partial t}-u(t) \frac{\partial n(\xi, t)}{\partial \xi}=r(t) f(\xi)\left[\alpha\left(l_{s}\right)-n(\xi, t)\right]-g(\xi) n(\xi, t)
$$


where $\xi$ represents the bond length with respect to the scaling factor $h$, which is defined as the maximum displacement of the myosin head at which attachment can occur. $n(\xi, t)$ represents the fraction attached cross-bridges with scaled bond-length $\xi$, which can be interpreted as the actin-myosin bond distribution function; $u(t)$ is the scaled shortening velocity of a half sarcomere; $r(t)$ is the activation factor depending on the calcium present in the muscle fiber; and $\alpha$ is the overlap factor of the filaments. $f(\xi)$ and $g(\xi)$ represent the rate parameters for attachment and detachment of myosin to actin. They are postulated to vary with the bond length $\xi$ as shown in figure 3.2.

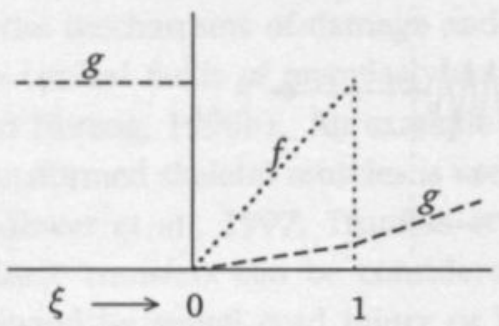

$$
\begin{aligned}
& f(\xi)= \begin{cases}0 & -\infty<\xi<0 \\
f_{1} \xi & 0 \leqslant \xi \leqslant 1 \\
0 & 1<\xi<\infty\end{cases} \\
& g(\xi)= \begin{cases}g_{2} & -\infty<\xi<0 \\
g_{1} \xi & 0 \leqslant \xi \leqslant 1 \\
g_{1} \xi+g_{3}(\xi-1) & 1<\xi<\infty\end{cases}
\end{aligned}
$$

Figure 3.2: Rate constants for attachment $(f)$ and detachment $(g)$ of myosin to actin. $\xi=1$ is the maximum displacement of the myosin head at which binding can occur.

In contrast to Ma and Zahalak (Ma, 1988; Zahalak and Ma, 1990; Zahalak and Motabarzadeh, 1997) we used simplified linear relations for $f(\xi)$ and $g(\xi)$ instead of the exponential relations that satisfy the thermodynamic equilibrium. From a mechanical point of view the differences are marginal (Gielen, 1998).

The overlap factor $\alpha$ is described (Herzog et al., 1992) as:

$$
\alpha(l)=\left\{\begin{array}{lll}
1-6.25(l-1)^{2} & l \leq 1 \\
1-1.25(l-1) & l>1
\end{array} \quad l=l_{s} / l_{s, o p t}\right.
$$

where $l_{s}$ and $l_{s, o p t}$ are the current and the optimal sarcomere length, respectively. The activation factor $r(t)$ describes the calcium activation in the Huxley cross-bridge model. A myosin head can only interact with an actin binding site if two calcium ions are bound to the specific receptor sites on the troponin molecule of that actin site (Zahalak and Ma, 1990). The fraction activated actin is defined as $r(t)$ and depends on the calcium present in the muscle (Zahalak and Ma, 1990; Zahalak and Motabarzadeh, 1997)):

$$
r(t)=\frac{[\mathrm{Ca}]^{2}}{[\mathrm{Ca}]^{2}+\mu[\mathrm{Ca}]+\mu^{2}}
$$


[Ca] represents the free calcium concentration in the myofibrilar space and $\mu$ the troponin-calcium reaction ratio constant. In order to determine the activation factor $r(t)$ the free calcium concentration $[\mathrm{Ca}]$ needs to be known. The net rate of the total calcium concentration $[\mathrm{Ca}]_{t}$ in the myofibrilar space equals the injection rate of calcium from the sarcoplasmatic reticulum (SR), minus the uptake rate of calcium into the SR:

$$
\frac{d}{d t}[C a]_{t}=[\dot{C} a]_{i n}-[\dot{C} a]_{u p}
$$

It is assumed (Zahalak and Ma, 1990) that each muscle action potential releases a fixed quantity of calcium with a fixed stereotyped time course. The calcium injection rate may be written as:

$$
[C a]_{i n}=R_{0}\left(1-\frac{[C a]}{[C a]^{*}}\right) \chi(t)
$$

where $R_{0}$ is the increase in total calcium concentration due to one action potential, $[\mathrm{Ca}]^{*}$ is the average calcium concentration in the muscle, and $\chi(t)$ is defined as the sum of normalized impulse functions. If the stimulation of the muscle starts at $t=0$, then $\chi(t)$ can be represented as:

$$
\chi(t)=\sum_{t_{i}} \hat{\chi}\left(t-t_{i}\right)
$$

in which $t_{i}$ is the time period between successive stimulation pulses,

$$
\hat{\chi}(t)=0 \text { for } t<0 \quad \text { and } \quad \int_{0}^{\infty} \hat{\chi}(t) d t=1 .
$$

The following analytical representation satisfies the conditions mentioned above:

$$
\hat{\chi}(t)=\frac{t}{\tau^{2}} e^{-t / \tau}
$$

with $\tau$ a time constant determining the time course of the calcium release.

The calcium injection rate has now been defined. The rate of calcium uptake into the SR is controlled by membrane-bound pumping proteins. According to Zahalak and Ma (1990) the classic Michaelis-Menten enzyme kinetics can be used to define the rate of calcium uptake as:

$$
[\mathrm{Ca}]_{u p}=V_{m} \frac{[\mathrm{Ca}]}{[\mathrm{Ca}]+K_{m}}
$$

where $V_{m}$ and $K_{m}$ are the two Michaelis-Menten parameters of the reaction. 
Finally the rate of the total calcium concentration in the myofibrilar space can be written as:

$$
[\mathrm{Ca}]_{t}=R_{0}\left(1-\frac{[\mathrm{Ca}]}{[\mathrm{Ca}]^{*}}\right) \chi(t)-V_{m} \frac{[\mathrm{Ca}]}{[\mathrm{Ca}]+K_{m}}
$$

The total calcium concentration in the myofibrilar space can now be determined at any time $t$, by solving differential equation 3.11. However, in order to determine the activation factor $r(t)$, the concentration of free calcium needs to be known. From the assumption of tight coupling and fast calcium-troponin equilibrium, Zahalak and Ma (1990) found an algebraic equation to relate the total calcium to the free calcium. $r(t)$ and the free calcium can be determined by using this additional algebraic equation:

$$
[C a]_{t}=[C a]+m^{*} b\left(2 Q_{0}+r(t)\left(2+\frac{\mu}{[C a]}\right)\left(\frac{1}{b}-Q_{0}\right)\right)
$$

where $m^{*}$ is the concentration of the cross-bridges (maximum possible), $b$ is referred to as the cross-bridge structural parameter (which is defined as $h / l_{a}$, where $l_{a}$ is the distance between two successive actin binding sites), and $Q_{0}$ the zeroth moment of the actin-myosin bond distribution function $n(\xi, t)$, defined as:

$$
Q_{0}=\int_{-\infty}^{\infty} n(\xi, t) d \xi
$$

\subsubsection{The distribution-moment (DM) approximation}

Since we are interested in the contractile behavior at regional rather than the crossbridge level, it suffices to approximate the solution of the Huxley equation by the socalled 'distribution-moment (DM) approximation method'. It was shown by Zahalak (1981) that by multiplying both sides of the Huxley equation by $\xi^{\lambda}(\lambda=0,1,2, \ldots)$ and by integrating this equation with respect to the scaled bond length variable $\xi$ $(=x / h)$, the Huxley equation becomes equivalent to an infinite system of coupled equations. Furthermore it was proposed by Zahalak (1981) to approximate $n(\xi, t)$ by a Gaussian distribution, which is determined by the first three moments $Q_{0}(t), Q_{1}(t)$ and $Q_{2}(t)$ of $n(\xi, t)$. The calculations reported in Zahalak (1981) result in a set of three coupled non-linear ordinary differential equations for the first three moments of $n$.

$$
\dot{Q}_{\lambda}=\alpha r \beta_{\lambda}-r \phi_{1 \lambda}\left(Q_{0}, Q_{1}, Q_{2}\right)-\phi_{2 \lambda}\left(Q_{0}, Q_{1}, Q_{2}\right)-\lambda u(t) Q_{\lambda-1}, \quad \lambda=0,1,2
$$

where the superimposed dot denotes time differentiation, and $Q_{\lambda}$ represents the $\lambda$-th normalized moment of the bond distribution function $n$ defined by

$$
Q_{1}=\int_{-\infty}^{\infty} \xi^{\lambda} n(\xi, t) d \xi, \quad \lambda=0,1,2
$$


The terms $\beta_{\lambda}, \phi_{1 \lambda}$ and $\phi_{2 \lambda}$ have been defined in Zahalak (1981). The three differential equations (equation 3.14 ) and the differential equation describing the net calcium rate (equation 3.11) define the complete DM activation contraction model, including calcium activation. An additional advantage of this DM model is that the first three moments of the bond distribution $n(\xi, t)$ have a physical meaning (Zahalak and Motabarzadeh, 1997; Zahalak and Ma, 1990; Zahalak, 1986). The first moment $Q_{1}$, for example, is proportional to the instantaneous active muscle force $F_{m}$

$$
F_{m}=\Gamma Q_{1}
$$

where the constant $\Gamma$ is referred to as the force scaling parameter.

\subsubsection{Distribution-Moment model for a muscle-tendon complex}

It is known from experimental data (Hatze, 1981; Hawkins and Bey, 1997) that the force-strain curves for tendons show an exponential course. Therefore the tendon is described by a non-linear exponential elastic spring:

$$
F_{t}=\frac{K}{s}\left(e^{\left(s \varepsilon_{t}\right)}-1\right)
$$

where $K$ can be interpreted as the initial resistance of the tendon against deformation, and $s$ determines the speed at which the resistance increases with increasing strain of the tendon. This strain is defined as

$$
\varepsilon_{t}=\frac{\left(Y-Y_{0}\right)}{Y_{0}}
$$

in which $Y$ is the length of the tendon, and $Y_{0}$ represents the initial length. During rest, at optimal length, the muscle-tendon complex is already pre-strained. If the prestrain of the tendon of the TA of the rat is defined as $\varepsilon_{0}$ the tendon force $F_{t}^{0}$ at rest equals

$$
F_{t}^{0}=\frac{K}{s}\left(e^{\left(s \varepsilon_{0}\right)}-1\right)
$$

So the tendon force of the actively contracting muscle equals:

$$
F_{t}=F_{t}^{0}+F_{m}
$$

Now let's consider the muscle as represented in figure 3.1 as a cylinder of active contractile tissue with length $X$, in series with a passive tendon of length $Y$. The total length of the muscle-tendon complex is defined as $L=X+Y$. To account for a finite tendon compliance, one more differential equation must be added to the DM equations 3.11 and 3.14. This fifth differential equation may be written as

$$
\dot{L}=\dot{X}+\dot{Y}
$$


The time derivative of $X$ and $Y$ are written (Zahalak, 1986) as:

$$
\begin{aligned}
& \dot{X}=-\left(\frac{2 h X_{0}}{l_{s 0}}\right) u(t) \\
& \dot{Y}=\frac{d Y}{d Q_{1}} \frac{d Q_{1}}{d t}
\end{aligned}
$$

Using equation $3.16,3.17,3.18$, and 3.20 we may write:

$$
\frac{d Y}{d Q_{1}}=\left(\frac{s}{Y_{0}}\left(Q_{1}+\frac{F_{t}^{0}}{\Gamma}\right)+\frac{K}{Y_{0} \Gamma}\right)^{-1}
$$

Now, the muscle stretch ratio, with respect to the initial length $L_{0}$ is defined as:

$$
\Lambda=\frac{L}{L_{0}}
$$

Further, two dimensionless geometric parameters $\theta$ and $\eta$ and a dimensionless compliance function $\kappa\left(Q_{1}\right)$ are defined as:

$$
\theta=\frac{X_{0}}{L_{0}}, \quad \eta=\frac{2 h}{l_{s 0}}, \quad \kappa\left(Q_{1}\right)=\frac{1}{L_{0}}\left(\frac{s}{Y_{0}}\left(Q_{1}+\frac{F_{t}^{0}}{\Gamma}\right)+\frac{K}{Y_{0} \Gamma}\right)^{-1}
$$

The fifth differential equation 3.21, that must be added to the DM equations 3.11 and 3.14 to account for the tendon compliance can finally be written as

$$
\dot{\Lambda}=\kappa\left(Q_{1}\right) \dot{Q}_{1}-\theta \eta u(t)
$$

\subsubsection{Summary}

The 1D model of the muscle-tendon complex consisting of a force generating contractile element (CE) and a series elastic element (figure 3.1) has been explained. The five first order coupled differential equations that embody this 1D model can be collected and are summarized below. By utilizing the normalized quantities

$$
\begin{array}{cccc}
C=[\mathrm{Ca}]_{t} / m^{*} & c=[\mathrm{Ca}] / m^{*} & C^{*}=[\mathrm{Ca}]^{*} / m^{*} & \rho=R_{0} / m^{*} \\
\tau_{0}=m^{*} / V_{m} & k_{m}=K_{m} / m^{*} & \mu=k_{-} / k_{+} m^{*} & b=h / l_{a}
\end{array}
$$

where $m^{*}$ represents the concentration of myosin heads and $l_{a}$ the distance between successive actin binding sites, the model may be written as

$$
\begin{aligned}
& \dot{Q}_{\lambda}=\alpha r \beta_{\lambda}-r \phi_{1 \lambda}\left(Q_{0}, Q_{1}, Q_{2}\right)-\phi_{2 \lambda}\left(Q_{0}, Q_{1}, Q_{2}\right)-\lambda u(t) Q_{\lambda-1}, \quad \lambda=0,1,2 \\
& \dot{C}=\rho\left(1-\frac{c}{c^{*}}\right) \chi(t)-\tau_{0}^{-1}\left(\frac{c}{c+k_{m}}\right)
\end{aligned}
$$




$$
\dot{\Lambda}=\kappa\left(Q_{1}\right) \dot{Q}_{1}-\theta \cdot \eta \cdot u(t)
$$

These equations were implemented in MATLAB which enables the solving of the system of differential equations by numerical integration using a Runga-Kutta integration scheme. By choosing the right initial values for the state variables $Q_{0}$, $Q_{1}, Q_{2}, C$ and $\Lambda$ and input values for the collection of model parameters, muscle contractions can be simulated.

\subsection{Parameter identification}

Most of the parameter values that appear in the five coupled differential equations embodying the 1D muscle model of the TA could be measured or taken from the literature (Ma, 1988; Hawkins and Bey, 1997) and are given in table 3.1.

Table 3.1: Parameter values of the DM model which could be measured or taken from the literature ${ }_{a}$ taken from Ma (1988); ${ }_{b}$ fitted to data from Hawkins and Bey (1997); c measured at dissected hind-limb of a rat; $d$ determined from Ma (1988).

\begin{tabular}{|c|c|c|c|c|c|c|c|c|}
\hline$\mu$ & $k_{m}$ & $b$ & $c^{*}$ & $K$ & $s$ & $\varepsilon_{0}$ & $\theta$ & $\eta$ \\
\hline $0.2_{a}$ & $0.006_{a}$ & $0.77_{a}$ & $1.0_{a}$ & $0.32_{b}$ & $60_{b}$ & $0.07_{b}$ & $3 / 5_{c}$ & $0.02_{d}$ \\
{$[-]$} & {$[-]$} & {$[-]$} & {$[-]$} & {$[N]$} & {$[-]$} & {$[-]$} & {$[-]$} & {$[-]$} \\
\hline
\end{tabular}

However, the values of the rate constants of attachment and detachment of the crossbridges, $f_{1}, g_{1}, g_{2}$ and $g_{3}$, are yet unknown for the TA of a rat and can not be measured directly. Three parameters associated with calcium activation are also unknown for the rats TA. These parameters are $\rho$, the calcium injection magnitude; $\tau_{0}$, the inverse maximum calcium uptake rate; and $\tau$, the time constant determining the duration of the calcium injection. Unlike the rate constants, these activation parameter values are constrained (Ma, 1988; Lehman, 1982). The values of the calcium injection magnitude $\rho$ found in literature lie between 2 and 5 (Ma, 1988). The reported values of the inverse maximum calcium uptake rate $\tau_{0}$ in different species varies from $0.0023 \mathrm{~s}$ to $2.3 \mathrm{~s}$ (Ma, 1988). The calcium injection pulse should last longer than $3 \mathrm{~ms}$ (Lehman, 1982) and shorter than $15 \mathrm{~ms}$ (Zahalak and Ma, 1990). Based on equation 3.9, this means a value for $\tau$ between $0.5 \mathrm{~ms}$ and $3.5 \mathrm{~ms}$. 
The parameter $\Gamma$ can be estimated from the maximum isometric fused tetanic force generated by the muscle $F_{m}^{(0)}$ at optimal muscle length via the relation:

$$
\Gamma=\frac{F_{m}^{(0)}}{Q_{1}^{(0)}}
$$

The value of $Q_{1}^{(0)}$ is the maximum value of $Q_{1}$ of a fused tetanus (high stimulation frequency) when the isometric constraint is imposed $(\dot{\Lambda}=0)$ and the overlap alpha is assumed to be 1 . However, the value of $Q_{1}^{(0)}$ depends on the parameter $\Gamma$ via the fifth differential equation 3.27 of the 1D muscle-tendon model. A sensitivity analysis has indicated that while the rest of the parameters remain unchanged, a change of $\Gamma$ from $40 N$ to $70 N$ (i.e. a $75 \%$ higher $\Gamma$ ) results in a $1 \%$ higher value for $Q_{1}^{(0)}$. Because the effect of $\Gamma$ on $Q_{1}^{(0)}$ during isometric contractions is very small, it is feasible to estimate $\Gamma$ from a calculated value of $Q_{1}^{(0)}$ using a first estimate for $\Gamma$ and the other unknown parameters and the maximum isometric force $F_{m}^{(0)}$. This force has been estimated from the experimentally determined muscle torques $\mathrm{T}$ of seven rats as described in the next section and the moment $\operatorname{arm} R$ of the TA. The moment $\operatorname{arm} R$ has been determined from a free dissected hind-limb of a rat and is approximately $2.8 \mathrm{~mm}$. This results in an estimate for the maximum isometric muscle force via the relation $F_{m}^{(0)}=T / R$ of $14.6 \pm 3.4 N$. From equation 3.29 the value of $\Gamma$ has been estimated to be $50 N$.

Identification of the unknown parameters requires experimental data of macroscopic muscle behavior (like muscle torque) to which model simulations can be compared. The parameter values can then be estimated by minimization of a least squares objective function:

$$
J(\theta)=[\underline{m}-\underline{s}(\underline{\theta})]^{T}[\underline{m}-\underline{s}(\underline{\theta})]
$$

with respect to the column $\underline{\theta}$ in which the unknown parameters of the $1 \mathrm{D}$ muscle model are stored. The measurements (muscle torques) are stored in a column $\underline{m}$ and the model simulations using a parameter set $\underline{\theta}$ are stored in $s(\underline{\theta}$.) The Gauss-Newton algorithm (Meuwissen, 1998) has been used to minimize the objective function. A necessary condition at a (local) minimum of $J(\underset{\sim}{\theta})$ is given by

$$
\left[\frac{\partial J(\underline{\theta})}{\partial \underline{\theta}}\right]^{T}=\underline{0}
$$

This results in a set of seven nonlinear equations for the seven unknown parameters

$$
\underline{H}^{T}(\underline{\theta})[\underline{m}-\underline{s}(\theta)]=0
$$

where the sensitivity matrix $\underline{H}(\underline{\theta})$ is given by

$$
\underline{H}(\underline{\theta})=\frac{\partial \underline{s}(\underline{\theta})}{\partial \underline{\theta}}
$$


Equation 3.32 has to be solved iteratively because the model response $s(\theta)$ is a nonlinear function of the parameters. An estimate of the optimal parameter set $\underline{\theta}^{(i)}$, where the index $i$ is the iteration counter, needs to be available. If the optimal parameter set is define as $\theta_{L S}$, then

$$
\underline{\theta}_{L S}=\underline{\theta}^{(i)}+\delta \underline{\theta}_{L S}^{(i)}
$$

Substitution of 3.34 into 3.32 for the optimum $\underline{\theta}_{L S}$ leads to

$$
\underline{H}^{T}\left(\underline{\theta}^{(i)}+\delta \theta_{L S}^{(i)}\right)\left[\underline{m}-\underline{s}\left(\underline{\theta}^{(i)}+\delta \theta_{L S}^{(i)}\right)\right]=0
$$

On the assumption that $\delta \theta_{L S}^{(i)}$ is small, the following two approximations can be made:

$$
\begin{aligned}
& \underline{s}\left(\underline{\theta}^{(i)}+\delta \underline{\theta}_{L S}^{(i)}\right) \approx \underline{s}\left(\theta^{(i)}\right)+\underline{H}\left(\theta^{(i)}\right) \delta \underline{\theta}_{L S}^{(i)} \\
& \underline{H}\left(\underline{\theta}^{(i)}+\delta \underline{\theta}_{L S}^{(i)}\right) \approx \underline{H}\left(\theta^{(i)}\right)
\end{aligned}
$$

Substitution of these approximations into equation 3.35 results in an iterative scheme for updating the parameters $\underline{\sigma}^{(i)}$

$$
\begin{aligned}
& \underline{\theta}^{(i+1)}=\underline{\theta}^{(i)}+\delta \tilde{\theta}^{(i)} \\
& \delta \theta^{(i)}=\underline{K^{i-1}}\left[\underline{H}\left(\tilde{\theta}^{(i)}\right)^{T}\left[\underline{m}-\underline{s}\left(\theta^{(i)}\right)\right]\right] \\
& \underline{K}^{(i)}=\underline{H}\left(\theta^{(i)}\right)^{T} \underline{H}\left(\theta^{(i)}\right)
\end{aligned}
$$

where $\delta \theta^{(i)}$ is an estimate of $\delta \theta_{L S}^{(i)}$. In the neighborhood of the optimal solution $\theta_{L S}$, this so called Gauss-Newton scheme has quadratic convergence.

\subsection{Experiments}

The experimental data were obtained by inducing isometric contractions of the TA muscle of seven male Wistar rats, during which the muscle torque $\mathrm{T}$ was measured using a rat isometric dynamometer (RIDM) (figure 3.3). A comparable set-up was also used to measure muscle torques of hindlimb flexor muscle complexes of intact mice. Gorselink et al. (2000) described this set-up in more detail. Isometric contractions are defined here as contractions where the total length of the muscle-tendon complex remains constant.

\subsubsection{Apparatus description}

The experimental set-up consists of 4 units (figure 3.3): a custom built rat isometric dynamometer (RIDM); an Apple Macintosh 7100 PowerPC with an 8 channel, 12 bits Lab-NB analog-to-digital conversion board (National Instruments ${ }^{\circledR}$, Mopac Expwy, Austin, USA) programmed with Lab-VIEW 3.1; a pulse generator for purpose 

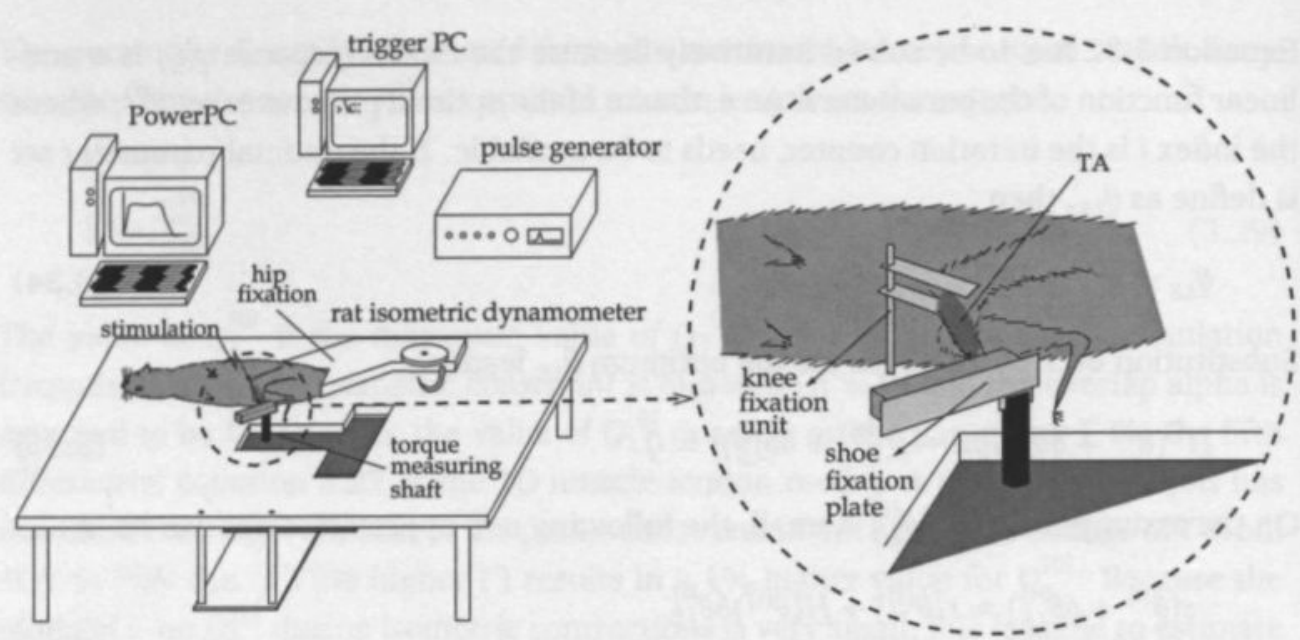

Figure 3.3: Experimental set-up of the rat isometric dynamometer (RIDM) for measuring muscle torques. The left part shows a rat in the RIDM and the 4 units of the set-up. The right part shows the fixation unit of the knee and the way the foot is fixed to the torque measuring axis in more detail.

of electrical stimulation (HSE 215/IZ, Freiburg, Germany); and a PC in order to trigger the stimulation and the data acquisition. During the experiment, the foot was rigidly connected to the shaft of the torque transducer. The torque measurement shaft was connected to two perpendicular placed custom-build leaf springs and a perpendicular placed beam. Muscle torque resulted in a minor rotation of the shaft, resulting in a slight displacement of the beam. The displacement of the beam was monitored by a displacement transducer. Assessments of muscle torque using the device presented here, ranged from $-220 \mathrm{Nmm}$ to $+220 \mathrm{Nmm}$ with an accuracy of $\pm 0.03 \mathrm{Nmm}$. Resonance frequency of the measurement device was $165 \mathrm{~Hz}$. The axis angle could be varied and was monitored with an optical encoder. Data acquisition was performed at $1000 \mathrm{~Hz}$. The measurement unit was calibrated with an external torque calibration unit connected to the thermostatic platform $\left(38.5 \pm 0.1^{\circ} \mathrm{C}\right)$. The calibration curve was acquired using precision weights at a well-defined distance from the torque unit axis. The calibration range was between $-220 \mathrm{Nmm}$ and $+220 \mathrm{Nmm}$, and calibration was performed with an increasing and decreasing series of weights at a well-defined distance $(7.1 \mathrm{~cm})$ from the torque unit axis. Calibration showed an excellent linear fit $(r>0.999)$ with a scaling factor of $250 \mathrm{Nmm} / \mathrm{V}$. The measuring noise during dynamic measurements was $0.5 \mathrm{Nmm}$. 


\subsubsection{Experimental procedure}

All experiments $(n=7)$ were performed on 12-week-old male Wistar rats (368 \pm 36gram, mean $\pm \mathrm{SD}$ ), positioned on an aluminum platform maintained at $38.5 \pm$ $0.1^{\circ} \mathrm{C}$. Initially the rats were anaesthetized by a sub-cutaneous injection of $0.15 \mathrm{ml}$ ketamine. From this moment on, halothane (Fluothane(r), Zeneca, Ridderkerk, The Netherlands) $(1.5-2.0 \%)$ in $\mathrm{O}_{2}$ and $\mathrm{N}_{2} \mathrm{O}\left(2: 1,3.0 \mathrm{~min} \mathrm{~m}^{-1}\right)$ was delivered via a face mask through a flow-meter system (Medec, Montvalle, N.J., USA). All experimental procedures were approved by the Institutional Animal Care and Use Committee of the Maastricht University and complied with the principles of proper laboratory animal care. After depilating the skin of the hind-limb of the anesthetized rat, a small incision in the hollow of the knee or in the lateral part of the knee was made to obtain access to the peroneal nerve. A bipolar platinum hook electrode, inter-pole distance $0.8 \mathrm{~mm}$, was carefully attached to the nerve, and the muscle complex could be stimulated using a pulse generator (HSE 215/IZ, Freiburg, Germany). In order to assure an isometric contraction of the TA, movement of the rats knee and foot had to be prevented. The foot was cast in a two-component cement (paladur, Heraus Kulzer GmbH, Wehrheim, Germany) shoe using a Teflon mould that was glued with cryano-acrylate (Bison, Goes, The Netherlands) to the shoe-fixation plate. This plate was connected to the apparatus shaft. The proximal part of the hind limb was fixed to the external world through a hip-fixation unit (figure 3.3). The rat was positioned sidelong to the hip-fixation unit and the tail was conducted through a small opening in the fixation platform. The hip was fixed to a vertical platform by winding the tail on a pulley. Tail friction, obtained by an external force $\left(F_{\text {tail }}=3 \mathrm{~N}\right)$ on the pulley, fixed the hip onto the vertical platform. The knee was supported by two aluminum plates to prevent lateral movement while permitting sagittal plane movements. Rigid knee fixation is not allowed, because an extra fixation force, as a result of prevention of sagittal knee movements, will be transmitted via the tibia as an eccentric force, introducing a non-muscular torque to the torque transducer. In a series of contractions, the angle of the rats ankle was optimized for maximal fused tetanic torque. This angle appeared to be about $90^{\circ}$. After this optimization the stimulation current was optimized for maximal fused tetanic torque. The pulse duration of the electrical stimulation current was $0.5 \mathrm{~ms}$ and the optimal stimulation current was about $1 \mathrm{~mA}$. After these preparations, the exercise protocol could be carried out.

\subsubsection{The exercise protocol}

The protocol consisted of contractions which were induced by $300 \mathrm{~ms}$ electrical stimulation at different stimulation frequencies: $40,50,67$ and $125 \mathrm{~Hz}$. Beside these 
contractions, a twitch stimulation was also given. A series of measurements was started with a twitch, followed by the different stimulation frequencies starting with the lowest frequency. For each rat, three such measuring series $(m=3)$ were carried out in order to test the reproducibility of the muscle torques. Between contractions, a rest period of three minutes was maintained in order to avoid fatigue. During the contractions the muscle torques were measured and in order to reduce the signal to noise ratio, the measurements were filtered using a 5 point zero-lag moving-average.

\subsection{Results}

For each rat the measured torques $T$ are considered relative to the maximum isometric torque $T_{m}$ at $125 \mathrm{~Hz}$. Also, the simulated first moments $Q_{1}$ of the distribution function $n(\xi, t)$, which are proportional to the muscle force and thus the muscle torque, are considered as a fraction of the maximum isometric first moment $Q_{1, m}$ at $125 \mathrm{~Hz}$. This normalization enables a direct comparison of the normalized measured torques to the normalized simulated first moments. Also, the normalized torques enable mutual comparison of measurements between different rats without having to concern about scaling differences.

For each rat and each stimulation frequency, the torque measurements were performed three times $(\mathrm{m}=3)$. The average measured muscle torque at a stimulation frequency of $125 \mathrm{~Hz}$ was $40.9 \pm 9.5 \mathrm{Nmm}$. The maximal difference relative to the maximum torque at $125 \mathrm{~Hz}$ between the three repeat torque measurements on the same rat were small $(5 \%)$ compared to the the maximal differences between measurements at different rats (40\%). Therefore in figure 3.4 only one set of torque measurements (black lines) is displayed for each rat. These measurements will also be used for parameter identification. Note that on the first rat the $40 \mathrm{~Hz}$ torque measurement was not performed.

The so-called optimal parameters were determined by minimization of the objective function (equation 3.30) as described section 3.3. By using the optimal parameter values, the isometric torques were simulated for each rat. In figure 3.4 , these simulation results are compared to the experimental results for each rat. The optimal parameter values for each rat are also presented in table 3.2.

Figure 3.4 shows a fairly good agreement between model and experiment at the different stimulation frequencies. However, some differences can be found. The most pronounced difference appears at the relaxation of the isometric contractions at a stimulation frequency of $125 \mathrm{~Hz}$ and, to a lesser degree at $67 \mathrm{~Hz}$. The simulated relaxation at these frequencies is much faster than that measured, while simulated relaxation of the twitch is generally slower than that obtained experimentally. 

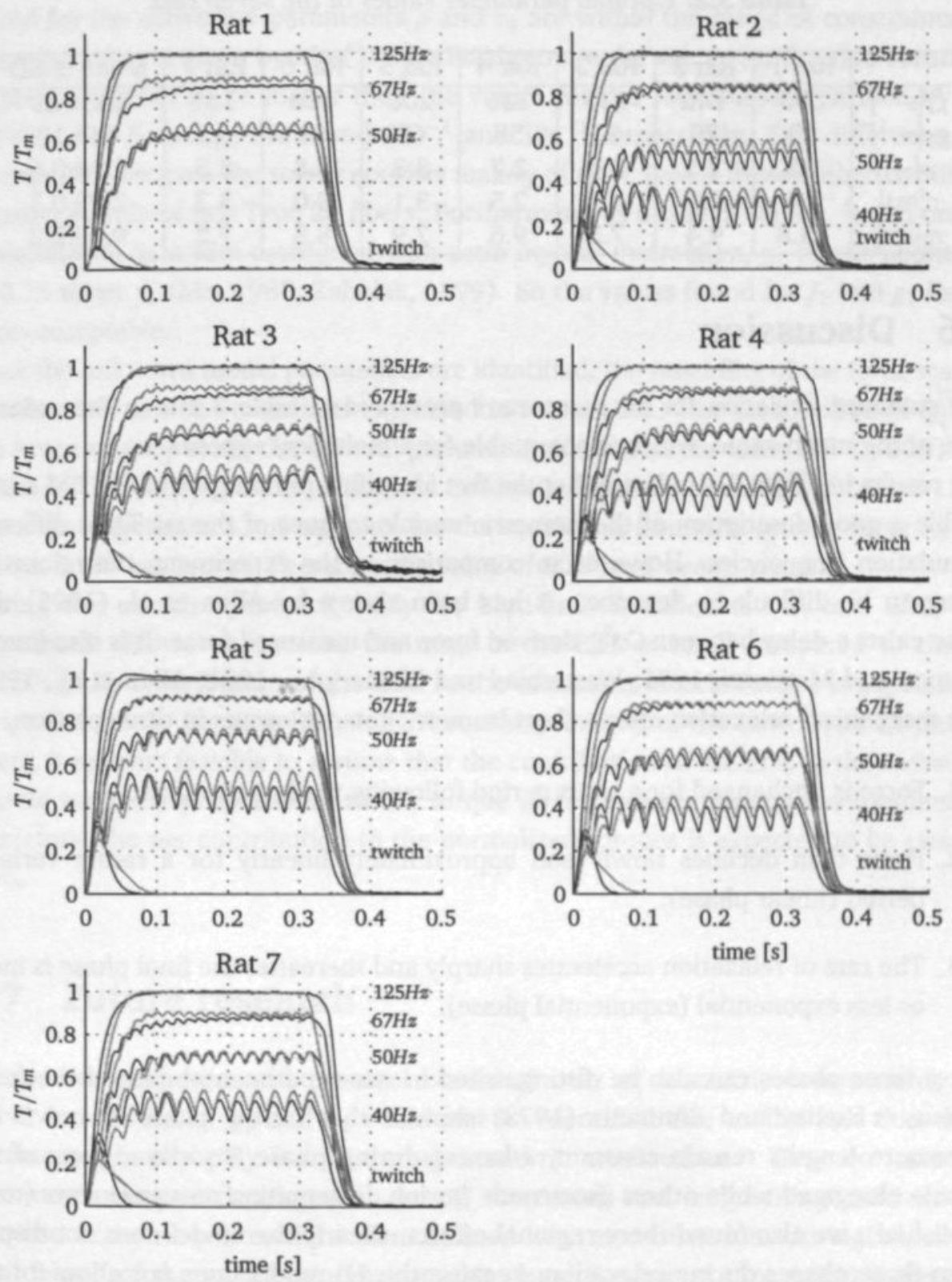

Figure 3.4: Measured (black lines) and simulated (gray lines) normalized isometric torques for each rat. The stimulation frequencies are indicated on the right. The optimal parameter values for each rat are displayed in table 3.2 . 
Table 3.2: Optimal parameter values of the seven rats.

\begin{tabular}{|c|c|c|c|c|c|c|c|c|}
\hline & Rat 1 & Rat 2 & Rat 3 & Rat 4 & Rat 5 & Rat 6 & Rat 7 & Mean \pm SD \\
\hline$f_{1}\left[s^{-1}\right]$ & 203 & 142 & 137 & 136 & 200 & 169 & 151 & $163 \pm 29$ \\
$g_{1}\left[s^{-1}\right]$ & 70 & 79 & 51 & 58 & 66 & 69 & 55 & $64 \pm 10$ \\
$\rho[-]$ & 1.8 & 2.5 & 3.4 & 2.7 & 3.3 & 2.8 & 3.5 & $2.9 \pm 0.6$ \\
$\tau[\mathrm{ms}]$ & 2.6 & 2.7 & 3.1 & 2.5 & 3.1 & 3.0 & 3.2 & $2.9 \pm 0.3$ \\
$\tau_{0}[\mathrm{~ms}]$ & 13.8 & 9.4 & 7.5 & 9.6 & 7.9 & 8.8 & 7.2 & $9.2 \pm 2.2$ \\
\hline
\end{tabular}

\subsection{Discussion}

The standard deviation for all parameters presented in table 3.2 is in the order of $20 \%$ of the mean value, which is acceptable for a biological experiment.

The results in figure 3.4 indicate that the five identified parameters of the DM model enable a good description of the isometric muscle torques of the rat TA at different stimulation frequencies. However, in comparison to the experiment, the relaxation seems to be difficult to describe. It has been shown by Allen et al. (1995) that there exists a delay between $\mathrm{Ca}^{2+}$-derived force and measured force. It is also known (Edman and Mattiazzi, 1981; Westerblad and Lännergren, 1991; Allen et al., 1995) that mechanical relaxation after a short isometric tetanus occurs in three phases.

1. Force is unchanged for a short period following the last stimulus.

2. Force then declines slowly and approximately linearly for a rather variable period (linear phase).

3. The rate of relaxation accelerates sharply and thereafter the final phase is more or less exponential (exponential phase).

These three phases can also be distinguished in our experimental data of the fused tetanus. Huxley and Simmons (1973) showed that during phase 1 and 2 the sarcomere lengths remain constant, whereas during phase 3 some regions of the muscle elongated while others shortened. During deformation measurements (to be published), we also found these regional effects. Clearly the model does not display these three phases during relaxation, because the 1D model does not allow for the regional effects. Concerning the $1 \mathrm{D}$ model, it was decided to describe the average relaxation behavior of the three phases by the two-state Huxley model. The identified parameters will be implemented in a continuum model which allows for regional effects and, thus, can be used to study regional deformation and the effect on the three relaxation phases. 
The values found for the DM parameters seem realistic in the sense that the values found for the activation parameters $\rho$ and $\tau_{0}$ are within the range of constraints for these parameters given by Ma (1988). Furthermore the values for the rate constants of the TA appear to be higher then the values for the rats soleus identified by Ma (1988). For $f_{1}$ and $g_{1}$ Ma found $90 s^{-1}$ and $30 s^{-1}$ respectively. This difference can be expected because the soleus consists mainly of slow Type 1 muscle fibers while TA consists mainly of fast Type $2 \mathrm{~b}$ fibers. Furthermore, as a rule of thumb, based on the maintenance heat rate associated with actin-myosin interaction, $g_{1}$ equals about 0.4 to 0.15 times $f_{1}$ (Ma, 1988; Zahalak, 1999). So the values found for $f_{1}$ and $g_{1}$ for TA seem acceptable.

Once the unknown model parameters are identified, the reliability of the force scaling parameter $\Gamma$ can be verified, as explained in section 3.3. The mean value of $Q_{1}^{(0)}$ of the seven rats, by using the optimal parameter values and the value of $\Gamma$ of $50 \mathrm{~N}$, was $0.338 \pm 0.027$. Using these values, the maximum isometric force $F_{m}^{(0)}$ is $16.9 \pm 1.4 N$. From the experiments, an estimate for the maximum isometric muscle force $F_{m}^{(0)}$ of $14.6 \pm 3.4 \mathrm{~N}$ was found. Therefore, he value of $50 \mathrm{~N}$ for $\Gamma$ seems reliable.

During the exercise protocol, both the hind-limb dorsiflexors (TA and EDL) are stimulated. However, because the mass of the EDL is small compared to the TA ( $\pm 20 \%$ ), the contribution of the EDL to the measured muscle torque is expected to be small as well. Because EDL and TA consist primarily of the same Type $2 \mathrm{~b}$ muscle fibers, it appears feasible to assume that the contribution of the EDL to the measured muscle torque is proportional to the torque at the various stimulation frequencies. Therefore, the net contribution to the normalized torques is expected to be close to zero.

\subsection{Future research}

Because the experimental protocol consisted only of isometric contractions, the parameter values of $g_{2}$ and $g_{3}$ could not be identified due to the fact that these parameters hardly play a role during isometric contractions. They do influence the muscle torques development during shortening and lengthening contractions. Because isometric contractions were defined here as contractions during which the total length of the muscle-tendon complex remains constant, one might expect the muscle tissue to shorten during these contractions. However, during deformation measurements (to be published) it appeared that the maximal global strain of the muscle tissue was less than $4 \%$, which confirms the results of Hawkins and Bey (1997). In the 1D model, the strain of the contractile element at maximal fused tetanus was also about $4 \%$ when using the identified parameters. By varying the 
parameters $g_{2}$ and $g_{3}$, the sensitivity of the parameters was tested during isometric contractions. It appeared that a change of $+50 \%$ or $-50 \%$ for each individual parameter $g_{2}$ and $g_{3}$ from its previous optimal value gave a difference less than $2.5 \%$ of the maximum fused torque for $g_{2}$, and less than $0.5 \%$ for $g_{3}$. So, for a proper identification of the rate constants $g_{2}$ and $g_{3}$, experimental data on shortening and lengthening contractions are needed. In the future we will collect experimental data under several experimental conditions. By comparing model simulations for different experimental conditions, the identified parameter values will be validated, and $g_{2}$ and $g_{3}$ will be identified.

The 1D model can be used to study the macroscopic behavior of the rat TA for isometric contractions and, after identification of $g_{2}$ and $g_{3}$, also for shortening and lengthening contractions. Furthermore, the identified parameters will be implemented in a 3D continuum model of the TA. For the time being, it will be assumed that the DM parameters are distributed homogeneously through the muscle tissue. In order to determine whether or not the model describes the real stresses and strains, the model needs to be validated. Due to the complexity of the $3 \mathrm{D}$ model, the numerical results are difficult to interpret. Therefore a stepwise approach is chosen with an increasing level of complexity incorporating different measuring techniques. The first validation experiments were focused on the DM contraction model of the muscle. This validation step was described here. To measure deformation at the muscle surface, 3D marker displacements will be measured during contraction by 3D video analysis (van Bavel et al., 1996; van Donkelaar et al., 1999b). Validation of the internal tissue deformation will be carried out by a MRI-tagging technique (Ossevoort, 1997; Kerckhoffs, 1998). During the validation steps, it will be investigated whether the assumption that the DM parameters are distributed homogeneously through the tissue is realistic. Special attention will also be paid to the sarcomere length distribution, the properties of the passive tissue and the ability of the 3D model to describe the three relaxation phases of the fused tetanic muscle torque. 


\section{Chapter 4}

\section{Superficial fiber stretch ratios}

of isometrically contracting muscle based on video analysis

M. Maenhout, M.K.C. Hesselink, P.J.B. Willems, C.W.J. Oomens, M.R. Drost

Effect of muscle force and ankle angle on the superficial fiber stretch ratio distribution of isometrically contracting rat tibialis anterior muscle, to be submitted to Journal of Biomechanics. 


\section{Abstract}

The objective of the present study is to measure in vivo local superficial stretch ratio distributions during electrical stimulation of an isometrically contracting tibialis anterior (TA) muscle of a rat $(n=7)$ in order to evaluate the dependence of the stretch ratio on 1) the muscle force and 2) the ankle angle. Superficial displacements were acquired by 3D video-analysis of surface markers, which were attached in muscle fiber direction from ankle to knee. The stretch ratios during the plateauphase calculated from the displacements were strongly dependent on longitudinal position of the markers. In the mid-area of the TA the stretch ratios were about 0.88 , and increased towards both muscle ends to about 1.05 . Less than $20 \%$ muscle force variation and small differences in the angle of the rats ankle (less than $5^{\circ}$ ) are shown not to affect local deformation. During onset and relaxation of the muscle force unexpected phenomena were observed. The most striking was that during force relaxation the distal regions showed additional shortening, while the proximal regions lengthened before the initial state was reached. The regional effects may be caused by the propagation of the action potential along the fibers originating from the motor end-plates which in the TA of the rat lies near the knee.

\subsection{Introduction}

As damage is initiated locally, data on local deformation are essential for proper understanding of the cause of muscle damage (Fitts et al., 1991; van der Meulen et al., 1993; Lieber et al., 1994; Koh and Herzog, 1998a). In successive contractions of a protocol to elicit damage, the muscle force generally declines, supposable due to fatigue. Declined contractile force is likely to affect the deformation during the course of the protocol. Furthermore, deformation may depend on the initial length of the muscle and thus on the joint angle.

Available deformation data like those reported by Hawkins and Bey (1997); Scott and Loeb (1995) involve only length changes of the whole muscle-tendon complex during contraction but no data on local changes. Stretch ratio distribution data during muscle contraction of intact muscles are taken from resting to fully contracting tissue (van Donkelaar et al., 1999b; Scott and Loeb, 1995; van Bavel et al., 1996). As far as the authors know local stretch ratio distributions along intact muscle fibers have only been reported at the plateau-phase at one stimulation frequency and ankle angle, so at one muscle force.

The objective of the present study is to measure in vivo local superficial stretch ratio distributions during electrical stimulation in order to evaluate the stretch ratio as a function of 1) the muscle force 2) the ankle angle and 3) time. 
The stretch ratios were acquired using 3D video analysis of markers which are attached to the exposed surface of the rat tibialis anterior (TA) muscle during an isometric contraction at optimal ankle angle and at maximal plantar flexion, both at 33 and $125 \mathrm{~Hz}$ stimulation frequency. The stretch ratio distribution is determined as a function of time, including onset and relaxation of the contractile force and the plateau-phase.

\subsection{Methods}

The experimental data were obtained by inducing isometric contractions of the tibialis anterior (TA) muscle of 7 male Wistar rats during which the muscle torque $\mathrm{T}$, which is proportional to the muscle force, was measured using a rat isometric dynamometer (RIDM, figure 4.1). A comparable set-up for mice has been described in detail by Gorselink et al. (2000). Superficial local fiber stretch ratios were measured by $3 \mathrm{D}$ video-analysis of surface markers. Isometric contractions are defined here as contractions where the total length of the muscle-tendon complex remains constant (i.e. no movement in the joint spanned by the muscles).
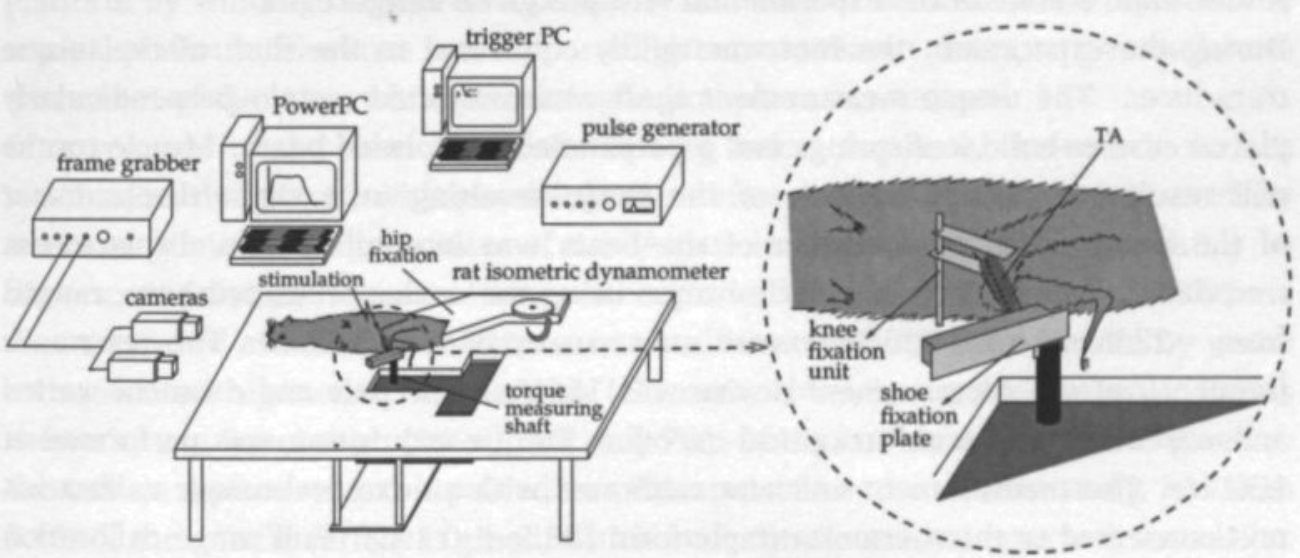

Figure 4.1: Experimental setup of the rat isometric dynamo-meter (RIDM) for measuring muscle torques. The left part shows a rat in the RIDM and the 4 units of the setup. The right part shows the fixation unit of the knee and the way the foot is fixated to the torque measuring axis in more detail.

\subsubsection{Apparatus Description}

The experimental setup consists of 5 units (figure 4.1), a custom built rat isometric dynamo-meter (RIDM), an Apple Macintosh 7100 PowerPC with an 8 channel, 
12 bits Lab-NB analog-to-digital conversion board (National Instruments ${ }^{\circledR}$, Mopac Expwy, Austin, USA) programmed with Lab-VIEW ${ }^{\circledR} 3.1$, a pulse generator for purpose of electrical stimulation (HSE 215/IZ, Freiburg, Germany) a PC in order to trigger the stimulation and the data acquisition and a marker detection system consisting of an 8-bit RGB frame-grabber (Magic RGB, Matrox, UK, 400*512 pixels) and two synchronized $25 \mathrm{~Hz}$ CCD cameras (MX5 Adimec, The Netherlands) with a 75-mm lens (Ernitec F1.3 TV). The frame-grabber was programmed with DMA Magic (DIFA measuring systems, Breda, The Netherlands) for real-time digitizing and storing video images $(25 \mathrm{~Hz})$ via the $\mathrm{R}$ and $\mathrm{G}$ input. The two cameras were placed on a platform at an angle between the optical axis of the cameras of $49.0^{\circ}$ such that the axis intersect in a central point in the workspace. The platform was positioned in front of the muscle. The line connecting the two lens centres was defined as the $x$-axis. The $y$-axis was perpendicular to the $x$-axis through the intersection point of the optical axis of the cameras. The $\mathrm{z}$-axis was perpendicular to the $\mathrm{x}$ - and $\mathrm{y}$-axis, and parallel to the longitudinal muscle axis. To optimize detection of the fluorescent markers, a blue filter (450 nm) was placed in front of the light source (KL 1500 electronic, Schot, Wiesbaden, Germany) and a yellow filter $(600 \mathrm{~nm})$ was placed in front of the cameras. A schematic outline of the experimental set-up is given in figure 4.1.

During the experiment, the foot was rigidly connected to the shaft of the torque transducer. The torque measurement shaft was connected to two perpendicularly placed custom-build leaf springs and a perpendicularly placed beam. Muscle torque will result in a minor rotation of the shaft, resulting in a slight displacement of the beam. The displacement of the beam was monitored by a displacement transducer. Assessments of muscle torque using the device presented here, ranged from $-220 \mathrm{Nmm}$ to $+220 \mathrm{Nmm}$ with an accuracy of $\pm 0.03 \mathrm{Nmm}$. The resonance frequency of the measurement device was $165 \mathrm{~Hz}$. The axis angle can be varied and was monitored with an optical encoder. Torque acquisition was performed at $1000 \mathrm{~Hz}$. The measurement unit was calibrated with an external torque calibration unit connected to the thermostatic platform $\left(38.5 \pm 0.1^{\circ} \mathrm{C}\right)$. Full range calibration was acquired by adding and removing precision weights at a distance of $7.1 \mathrm{~cm}$ from the torque unit axis. Calibration showed an excellent linear fit $(r>0.999)$ with a scaling factor of $250 \mathrm{Nmm} / \mathrm{V}$. The measuring noise during dynamic measurements was $\pm 0.5 \mathrm{Nmm}$.

\subsubsection{Experimental procedure}

Seven 12 week old male Wistar rats $(368 \pm 36 \mathrm{gram}$ (mean \pm standard deviation) were anesthetized by a sub-cutaneous injection of $0.15 \mathrm{ml}$ ketamine. Subsequently halothane (Fluothane(r), Zeneca, Ridderkerk, The Netherlands) $(1.5-2.0 \%)$ in $\mathrm{O}_{2}$ 
and $\mathrm{N}_{2} \mathrm{O}\left(2: 1,3.0 \mathrm{~min}^{-1}\right)$, was delivered via a face mask through a flow meter system (Medec, Montvalle, N.J., USA). All experimental procedures were approved by the Institutional Animal Care and Use Committee of the Maastricht University and complied with the principles of proper laboratory animal care. During the preparation and the experiment the rat was placed on an aluminum platform maintained at 38.5 $\pm 0.1^{\circ} \mathrm{C}$.

After depilating the skin of the left hind-limb of the anesthetized rat, a small incision in the lateral part of the knee was made to obtain access to the peroneal nerve. A bipolar platinum hook electrode, inter-pole distance $0.8 \mathrm{~mm}$, was carefully attached to the nerve for electrical nerve stimulation. In order to assure an isometric contraction, movement of the knee and foot had to be prevented. The foot was cast in a twocomponent cement (paladur, Heraus Kulzer GmbH, Wehrheim, Germany) shoe using a Teflon mould and was glued to the shoe-fixation plate with cryano-acrylate (Bison, Goes, The Netherlands). This plate was connected to the apparatus shaft. The proximal part of the hind limb was fixated to the external world through a hip-fixation unit (figure 4.1). The rat was positioned sidelong to the hip-fixation unit and the tail was conducted through a small opening. The hip (ischium) was fixated to a vertical platform by winding the tail on a pulley. A tail force of $3 \mathrm{~N}$ fixated the hip onto the vertical platform. The knee was supported by two aluminum plates, to prevent lateral movement of the knee, while permitting sagital plane movements.

After removal of the skin of the left hind leg below the knee, the muscle surface was frequently sprayed with physiological saline solution. The fascia was carefully removed from the medial surface of the TA muscle. Three rows of approximately 10 fluorescent polystyrene speres (Bangs Laboratories Inc. Fisher, USA) of $0.5 \pm 0.05 \mathrm{~mm}$ diameter were attached to the tissue in the direction of one fibre bundle by visual estimation with histoacryl tissue glue (Histoacryl blau,Braun, Melsungen). It was shown by van Bavel et al. (1996) that only $70 \%$ of the marker diameter was glued to the muscle surface with this procedure. Extrapolated to the present study, less than $0.1 \%$ of the muscle surface is glued, which unlikely affects total muscle deformation. The spheres were uniformally distributed in one row on the median area of the TA muscle with an inter-distance of $\sim 2 \mathrm{~mm}$. By gently pulling each marker and observing underlying tissue deformation under a stereo microscope, it was verified whether the marker was glued on muscle tissue and not on sparse remains of the fascia, which would severely affect the deformation (Scott and Loeb, 1995).

\subsubsection{The exercise protocol}

In a series of contractions, the angle of the rats ankle was determined for maximum fused tetanic torque. This angle appeared to be about $90^{\circ}$. The pulse duration of the 
electrical stimulation current was $0.5 \mathrm{~ms}$ and the current was determined from $100 \mathrm{~ms}$ contractions for maximum tetanic torque and was $1 \mathrm{~mA}$ for all rats.

The exercise protocol consisted of 12 contractions which were induced by $300 \mathrm{~ms}$ electrical stimulation. First a total of six contractions were carried out at alternating stimulation frequency of $33 \mathrm{~Hz}$ and $125 \mathrm{~Hz}$ at the optimal ankle angle $\left(\sim 90^{\circ}\right)$. A comparable series was performed at maximum plantar flexion (ankle angle $\sim 120^{\circ}$ ). So three replicates were measured at each combination of the experimental conditions. Between contractions a rest period of three minutes was maintained in order to avoid fatigue. Muscle torques and marker displacements were recorded during $700 \mathrm{~ms}$ starting $150 \mathrm{~ms}$ prior to contraction. In between contractions the stimulation frequency was changed between $33 \mathrm{~Hz}$ and $125 \mathrm{~Hz}$. The torque measurements were filtered by using a 5 point zero-lag moving-average.

\subsubsection{Data processing}

Geometric marker centroids were determined from the digitized image frames using TIMwin 1.3 (TEA Dordrecht, 1993). Three-dimensional marker tracks, i.e. three-dimensional positions for each marker in time were reconstructed from these centroids. Therefore marker tracks per camera were determined from the marker coordinates from the individual images (Muijtjens et al., 1997). Stereoscopic pairs of tracks in the two views were identified on the basis of minimum stereoscopic mismatch (Muijtjens et al., 1995b). Finally the geometry of the stereoscopic camera set-up was determined from these pairs and the corresponding three-dimensional marker tracks were reconstructed (Muijtjens et al., 1995a). Because all the markers were continuously visible in both the cameras the tracks from all applied markers could be reconstructed. The marker centroids in the images were calculated from either an odd or even image frame. Consequently the time between two subsequent marker positions was $20 \mathrm{~ms}$. A pixel-intensity weighted procedure was used increasing accuracy to $1 / 10$ th pixel, which corresponds to $\sim 10 \mu \mathrm{m}$.

Stretch ratios defined as

$$
\lambda=\frac{l_{a}}{l_{0}}
$$

were computed from the displacements of marker-pairs in the central row of the muscle, further denoted as regions. $l_{0}$ represents the initial length of a region recorded approximately $150 \mathrm{~ms}$ prior to the contraction and $l_{a}$ is the actual length. A region consisted of two nearest markers in the central band of the muscle. Consequently the computed stretch ratios were approximately in the fiber direction. The accuracy of data processing, from the rough image acquisition to the stretch 
ration calculation is within 0.005 . This was estimated from computations at marker positions between two subsequent odd fields of a resting muscle.

\subsubsection{Statistics}

All data are given in mean \pm standard deviation.

Two-tailed t-tests were used to test the differences at the plateau phase of the mean of the muscle torque replicates between experimental conditions, as well as the mean differences of the means of the absolute values of the stretch ratio replicates minus one $(|\lambda-1|)$. This absolute value represents the level of deformation. Differences were considered significant at $p<0.05$.

To exclude possible dependence of the differences in the stretch ratio on the position the squared correlation coefficient was calculated. If $r^{2}<0.01$ it was assumed that the difference in stretch ratio did not depend on the position. Non-linear dependence was evaluated visually.

\subsection{Results}

The standard deviation of the three torque replicates at optimal ankle angle and at a stimulation frequency of $125 \mathrm{~Hz}$ was $0.6 \pm 0.4$, which is about ten times smaller than the variation between rats (table 4.1). The standard deviation of the stretch ratio replicates at optimal ankle angle and at a stimulation frequency of $125 \mathrm{~Hz}$ was $0.005 \pm 0.001$, which is also much smaller than the variation between rats as will become clear in the following paragraphs.

Table 4.1: Mean values of the mean of the torque replicates during the plateau phase of seven rats at different experimental conditions.

\begin{tabular}{|c|cc|}
\hline $\begin{array}{c}\text { Stimulation } \\
\text { frequency }\end{array}$ & $\begin{array}{c}\text { Torque at optimal } \\
\text { ankle angle [Nmm] }\end{array}$ & $\begin{array}{c}\text { Torque at max. } \\
\text { plantar flexion [Nmm] }\end{array}$ \\
\hline $125 \mathrm{~Hz}$ & $36.7 \pm 5.3$ & $30.1 \pm 4.6$ \\
$33 \mathrm{~Hz}$ & $29.6 \pm 4.9$ & $27.2 \pm 4.8$ \\
\hline
\end{tabular}

Figure 4.2 shows the stretch ratio distribution during the plateau-phase at optimal ankle angle and the difference in stretch ratios between optimal ankle angle and maximum plantar flexion (stimulation frequency $125 \mathrm{~Hz}$ ). The mean difference in the muscle torques during the plateau-phase of the seven rats was not significant (table 4.2). The mean stretch ratios minus one of the muscle stimulated at $125 \mathrm{~Hz}$ at optimal ankle angle were significantly higher than during plantar flexion, as shown in table 
Table 4.2: Mean values of the differences at the plateau phase of the mean of the muscle torque replicates between experimental conditions of the seven rats, as well as the mean differences of the means of the absolute values of the stretch ratio replicates minus one $(\lambda \lambda-1 \mid)$. Statistical differences are indicated by $*(p<0.05)$.

\begin{tabular}{|c|c|c|}
\hline & $\Delta T[\mathrm{Nmm}]$ & $\Delta(|\lambda-1|)[-]$ \\
\hline $\begin{array}{c}\text { Optimal ankle angle } \\
125-33 \mathrm{~Hz}\end{array}$ & $\begin{array}{c}7.2 \pm 0.9 \\
*\end{array}$ & $5.3 \cdot 10^{-5} \pm 5.5 \cdot 10^{-3}$ \\
\hline $\begin{array}{c}\text { Plantar flexion } \\
125-33 \mathrm{~Hz}\end{array}$ & $\begin{array}{c}4.4 \pm 2.1 \\
*\end{array}$ & $9.4 \cdot 10^{-5} \pm 6.6 \cdot 10^{-3}$ \\
\hline $\begin{array}{c}125 \mathrm{~Hz} \\
\text { optimal - plantar }\end{array}$ & $5.1 \pm 6.3$ & $1.1 \cdot 10^{-2} \pm 6.3 \cdot 10^{-3}$ \\
\hline $\begin{array}{c}33 \mathrm{~Hz} \\
\text { opimal - plantar }\end{array}$ & $3.2 \pm 4.1$ & $\begin{array}{c}1.2 \cdot 10^{-2} \pm 4.6 \cdot 10^{-3} \\
*\end{array}$ \\
\hline
\end{tabular}

4.2. This implies that the contracting muscle deformed more at optimal lenght, than at plantar flexion. The differences in torques and stretch ratios between the two joint angles at a stimulation frequency of $33 \mathrm{~Hz}$ were comparable (table 4.2).

a

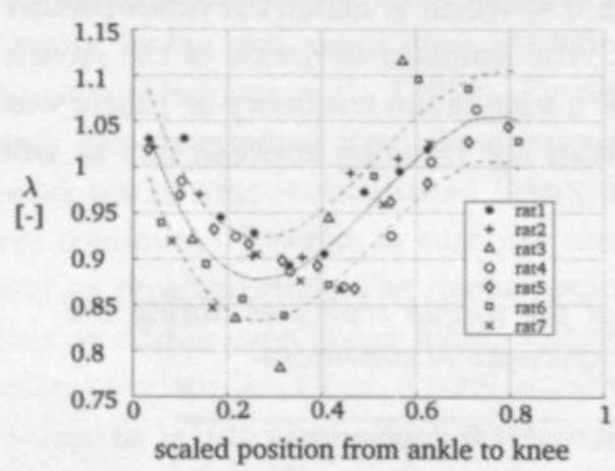

b

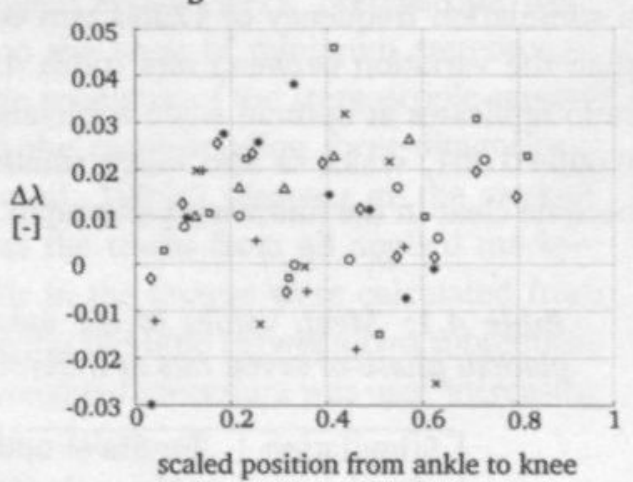

Figure 4.2: a) Distribution of the means of the stretch ratio replicates at $125 \mathrm{~Hz}$ and optimal length, during the plateau-phase with a least square fit and $50 \%$ error bounds. b) Differences of the means of the stretch ratio replicates between optimal ankle angle and maximum plantar flexion at $125 \mathrm{~Hz}$ stimulation frequency. The standard deviations of the mean replicate values were not larger than the size of the markers. 
Muscle torques during the plateau-phase was significantly higher after $125 \mathrm{~Hz}$ stimulation than after stimulation with $33 \mathrm{~Hz}$ (table 4.2). However, the mean stretch ratios were similar at $125 \mathrm{~Hz}$ and $33 \mathrm{~Hz}$ (table 4.2). Therefore, the stretch ratio distribution for the $33 \mathrm{~Hz}$ stimulation frequency are not shown. The differences in torques and stretch ratios between the two stimulation frequencies during plantar flexion were comparable (table 4.2).

a

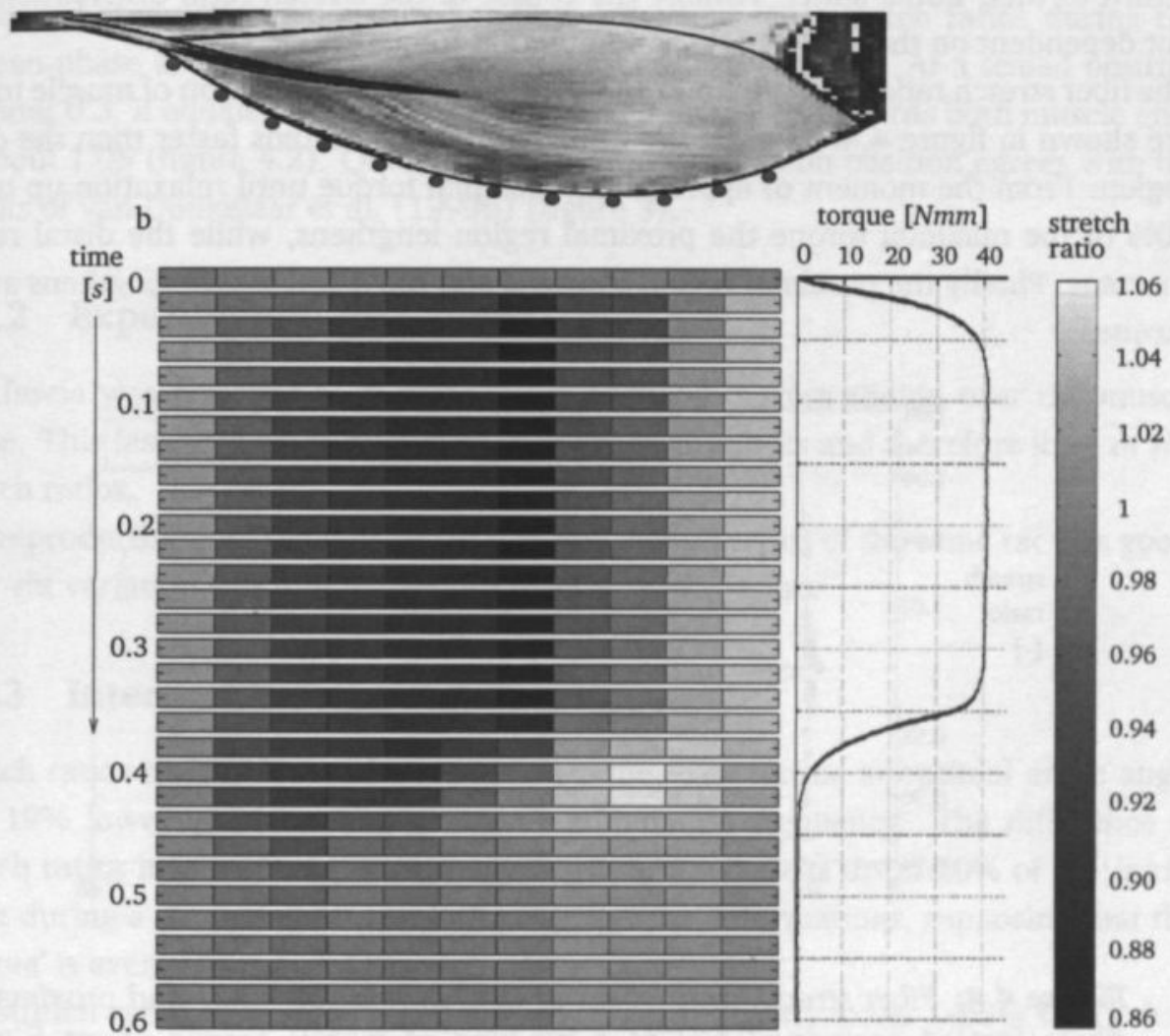

$0 \longrightarrow$ scaled position from ankle to knee 0.85

Figure 4.3: a) MR image of the mid-sagittal cross section of a TA muscle of a rat, illustrating the marker positions from which the fiber stretch ratios were calculated. b) Superficial stretch ratio distribution and muscle torque (one replicate) in time of rat 5 at optimal ankle angle and $125 \mathrm{~Hz}$ stimulation frequency.

The evaluated differences in stretch ratios as a function of the position in the muscle did not indicate a non-linear relationship and the calculated squared correlation coefficient was less than 0.01 for all cases. 
In figure 4.3 the course of the stretch ratio distribution and the measured muscle torque at optimal ankle angle are shown. During the plateau-phase the regions at the muscle ends are elongated (stretch ratio up to 1.03), while the regions in the mid-belly are shortened (stretch ratio up to 0.86 ). This distribution is reached within the first 20 to $40 \mathrm{~ms}$. During torque relaxation the stretch ratios remain constant for a short period, after which the distal regions show additional shortening, while the proximal regions lengthen (stretch ratio up to 1.07). Subsequently all regions return to their initial state. Visually the course of the stretch ratio distribution was not dependent on the ankle angle or stimulation frequency.

The fiber stretch ratios of a proximal and a distal region as a function of muscle torque are shown in figure 4.4. Initially the proximal region shortens faster than the distal region. From the moment of approaching maximal torque until relaxation up to 10$20 \%$ of the maximal torque the proximal region lengthens, while the distal region shortens. Finally the proximal region shortens and the distal region lengthens at low torque.

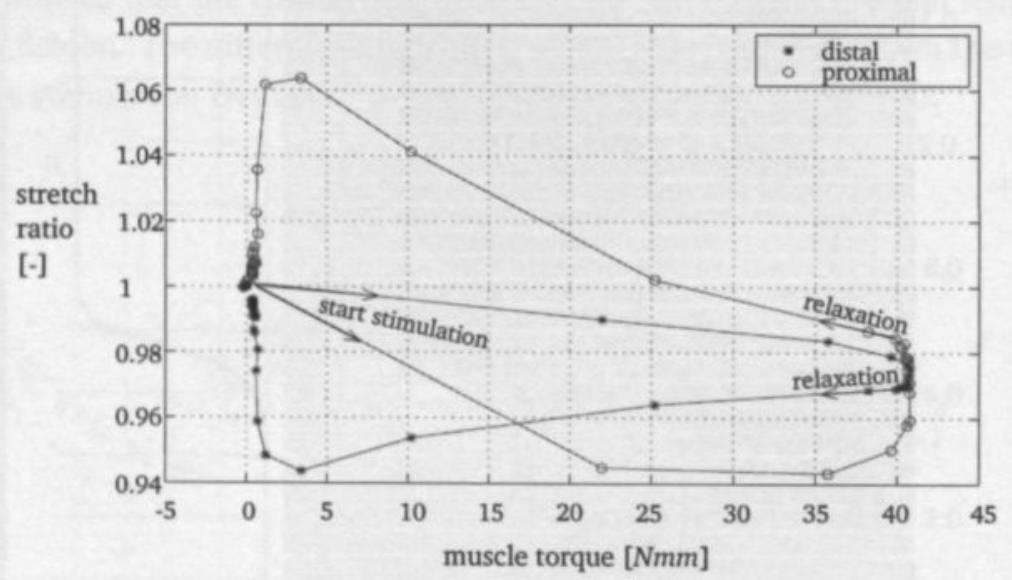

Figure 4.4: Fiber stretch ratio of distal (scaled position=0.1) and proximal fibers (scaled position $=0.63$ ) as a function of muscle torque of rat 5 at optimal length and $125 \mathrm{~Hz}$ stimulation frequency (one replicate). 


\subsection{Discussion}

\subsubsection{General}

This paper presents the experimentally acquired superficial muscle fiber stretch ratio distribution as a function of time at two different stimulation frequencies and ankle angles. To the best of the authors knowledge this is the first time that the relation between local deformation of contracting muscle tissue and the time courses, ankle angle and stimulation frequency has been reported. The stretch ratios during the plateau-phase are strongly dependent on longitudinal position. At a scaled position of about 0.3 , it equaled approximately 0.88 , and increased towards both muscle ends to about 1.05 (figure 4.2). Qualitatively this dependence on position agrees with the results of van Donkelaar et al. (1999b) (figure 3).

\subsubsection{Experimental procedure}

The fascia was removed to prevent attached markers from sliding over the muscle tissue. This fascia might restrict local increase in thickness and therefore limit in situ stretch ratios.

The reproducibility of the muscle torques and stretch ratios of the same rat was good. Inter-rat variation was about $10 \%$ of the intra-rat variation.

\subsubsection{Interpretation of results}

Stretch ratios did not depend on muscle torque. The torque at optimal ankle angle was $19 \%$ lower at $33 \mathrm{~Hz}$ than at $125 \mathrm{~Hz}$ stimulation frequency. The difference in stretch ratios however was negligible. Thus, torque deficits up to $20 \%$ of the initial value during a damaging protocol do not affect the deformations, supposing that the 'fatigue' is evenly distributed throughout the muscle.

The stretch ratios at optimal ankle angle were significantly lower (about 0.01) than the stretch ratios during plantar flexion, while the muscle torques during the plateauphase did not differ significantly. However, the differences are relatively small $(0.01$ compared to a stretch ratio range in logitudinal direction of 0.17 ) and the distribution of the stretch ratios was not influenced by the muscle length. Thus small differences in the initial angle of the rats ankle during a damaging protocol are not supposed to have great influence on local deformation.

During onset and relaxation of the muscle torque unexpected phenomena were observed. At the start of the stimulation, while the torque increased, proximal regions shortened faster than distal regions (figure 4.4). During torque relaxation the stretch ratios remained constant for a short period, after which the distal regions showed 
additional shortening, while the proximal regions lengthened (stretch ratio up to 1.07 , figure 4.4 and 4.3 ). Finally all regions went back to their initial state. These phenomena were observed in all the rats. The regional effects are also shown by Huxley and Simmons (1973) for sarcomeres in a single muscle fiber. The time scale in which the regional effects happen and in which the action potential propagates along the muscle fibers from proximal to distal ( $30 \mathrm{~ms}$, (Ganong, 1981; Bernards and Bouman, 1996; Guyton and Hall, 2000)) are the same. This implies that the effects may be caused by the propagation of the action potential along the fibers originating from the motor end-plates. Because the proximal regions shortened faster at the start of the stimulation and showed lengthening during torque relaxation, the motor end-plates are supposed to be situated proximally. A double immunofluorescence assay was performed on sections of a rat TA with $\alpha$-bungarotoxine and an antibody against fibronectin. Nuclei were visualized using DAPI. From immunofluorescence (IF) microscopic pictures, as shown in figure 7.1 (chapter 7), it became clear that the motor end-plates are indeed situated near the knee at a scaled position of about 0.7. The hypothesis that the propagation of the action potential along muscle fibers strongly influences the course of local strain distribution can be investigated in future by incorporating inhomogeneous activation of the muscle fibers in a $3 \mathrm{D}$ continuum model of the rat TA muscle. 


\section{Chapter 5}

\section{D strain fields within isometrically contracting muscle based on MR tagged images}

M. Maenhout, E.L.A. Blezer, M.K.C. Hesselink, A. van der Toorn, K. Nicolay, C.W.J. Oomens, E.P. Baaijens, M.R. Drost

Three-dimensional strain fields of isometrically contracting rat tibialis anterior muscle based on MR tagged images, submitted to Magnetic Resonance in Medicine. 


\section{Abstract}

The aim of this paper is to determine the sagittal and transverse strain gradients in contracting muscle to evaluate homogeneity of muscle deformation. Therefore 3D voxel by voxel displacement maps were acquired from two sets of orthogonal MR images with tissue tagging of isometrically contracting tibialis anterior (TA) muscle of a rat $(n=3)$. From the displacement fields 3D Green-Lagrange strains and strain gradients were derived. Longitudinal strain varied consistently over the muscle length with a maximum just distal from the midbelly of approximately +0.03 . The strain decreased in proximal and distal direction to approximately -0.08 . The variation per $\mathrm{mm}$ of the strains in sagittal and transverse direction was about $50 \%$ of the maximal strain. Therefore, superficial strains are not a good measure for deep strains. The maximal absolute transverse strain gradients were in the same order as the maximal absolute sagittal gradients $\left(0.1 \mathrm{~mm}^{-1}\right)$. This implies that deformation in the transverse direction is not negligible compared to the other two directions. The implication of the significant contribution of transverse deformation to contraction induced deformation is that, to simulate the distribution of mechanical quantities within a muscle, 2D models should be extended towards a realistic 3D geometry.

\subsection{Introduction}

Due to the local occurrence of adaptation (Koh and Herzog, 1998a; Stauber, 1989; Ebbeling and Clarkson, 1989; Fitts et al., 1991; van der Meulen et al., 1993; Lieber and Fridén, 1993; Lieber et al., 1994), knowledge of local muscle loading is important in studies towards understanding of muscle adaptation and damage. To study the local muscle loading numerical models of contracting muscle have been developed (Gielen et al., 2000; Vankan et al., 1998; van der Linden et al., 1998b; van Leeuwen and Spoor, 1996), which incorporate the assumption of constant muscle volume during contraction and represent 2D sagittal plane geometries.

The validity of the models is still uncertain due to limited experimental in vivo data concerning the distribution of mechanical quantities in contracting muscle tissue. The only data available are superficial strain distributions (van Donkelaar et al., 1999b; van Bavel et al., 1996). These distributions were acquired by 3D video analysis of markers which were superficially attached to the muscle. The results indicated that the transverse superficial strains were not negligible with respect to the longitudinal strains (van Donkelaar et al., 1999b). To the authors knowledge, data of deep strains have not yet been published.

Recent developments in the field of cardiac deformation offer a technique to acquire non-invasively accurate 3D in vivo displacement fields (Kuijer et al., 2000; Thomas 
and McVeigh, 1997; Alistair and Young, 1996) from two orthogonal sets of MR images with tissue tagging. In these studies discrete displacement maps were obtained at the location of the tags. An easy and reliable method was used, increasing the resolution to $2 \mathrm{D}$ pixel by pixel displacement mapping. In human cardiac MRI the cyclic nature of the contractions (order $1 \mathrm{~Hz}$ ) is advantageously used in the imaging sequence.

The aim of this paper is to determine 1) the sagittal strain gradient to evaluate the suitability of superficial strains as a measure of deep strains and 2) the transverse strain gradient to evaluate the suitability of sagittal plane models to describe the distribution of mechanical quantities within the muscle tissue.

A method was developed to non-invasively acquire in vivo $3 \mathrm{D}$ voxel by voxel displacement maps during skeletal muscle contraction based on methods to determine echo shifts in ultrasound pulsed Doppler instruments (Jong et al., 1990) and in phase-based optical flow (Jahne, 1993). The method was applied to acquire 3D displacement maps of steady-state isometric contractions of the tibialis anterior (TA) muscle of the rat. The advantage of cyclic motion in cardiac imaging could not be used, since skeletal muscle will fatigue during repetitive contractions, resulting in a contractile force drop which will likely affect deformation. From the displacement data 3D strain maps, sagittal and transverse strain gradients and volume change maps were computed. Volume change was used to evaluate the reliability of the strains.

\subsection{Methods}

\subsubsection{Experimental setup}

Three male, 8 weeks old Wistar rats were used. They were anaesthetized with a mixture of 1 volume hypnorm $(0.315 \mathrm{mg} / \mathrm{ml}$ fentanyl citrate, $10 \mathrm{mg} / \mathrm{ml}$ fluanisone), 1 volume dormicum $(5 \mathrm{mg} / \mathrm{ml}$ midazolam hydrochloride) and 2 volumes distilled water (total volume $0.3 \mathrm{ml} / 100 \mathrm{gram}$ BW, i.p) and atropine $(0.025 \mathrm{ml} / 100 \mathrm{gram}$ BW, subcutaneous) after short term sedation $(<20 s)$ with $1 \%$ halothane in $\mathrm{N}_{2} \mathrm{O} / \mathrm{O}_{2}(70 / 30)$. Next, the rat was intubated and mechanically ventilated with $1.5 \%$ halothane in $\mathrm{N}_{2} \mathrm{O} / \mathrm{O}_{2}(70 / 30) . \mathrm{CO}_{2}$ was continuously monitored. The rat was placed on a heated water mattress to maintain a core temperature of $37^{\circ} \mathrm{C}$. The local ethical committee approved the experiments.

The foot was cast in a two-component cement (Paladur, Heraus Kulzer GmbH, Wehrheim, Germany) using a teflon mould and was rigidly glued with cryano-acrylate (Bison, Goes, The Netherlands) to the foot-plate (figure 5.1). The femur was fixed with a pair of arnite tweezers. The foot-plate was connected to the animal cradle with a low friction hinge. A thread of aramid fibers connected the foot-plate $(18 \mathrm{~mm}$ 


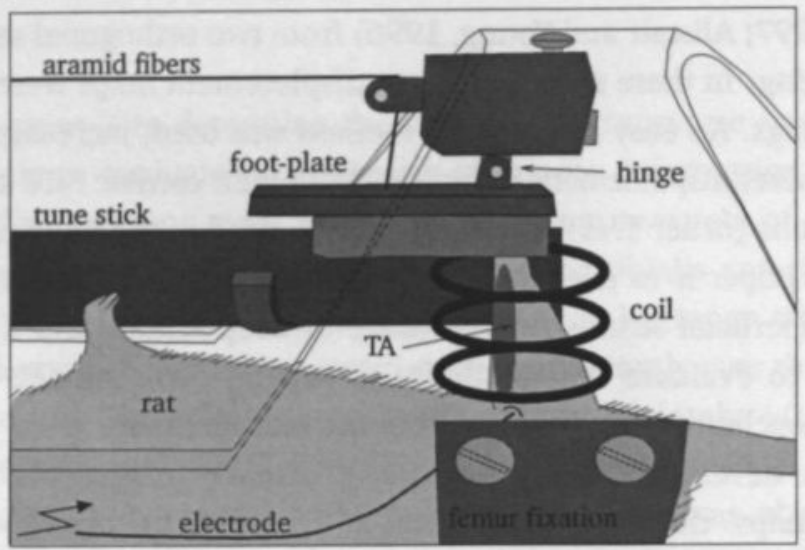

Figure 5.1: Experimental fixation device in the cradle. The femur of the rat is clamped between a pair of arnite tweezers and the plastered foot is glued to the foot-plate which is connected to a force transducer by aramid fibers. A solenoidal $R F$ coil with three turns was placed around the rats leg.

in front of the hinge) to a load cell (figure 5.1). The load cell was fixed to the cradle at a distance of about $30 \mathrm{~cm}$ from the rat and consisted of a stiff composite beam with a strain gauge attached to it. Calibration of the load cell showed an excellent linear fit ( $r>0.999)$ with a scaling factor of $26.9 \mathrm{~N} / \mathrm{V}$. A three-turn solenoidal RF coil was placed around the lower right hind-leg. The angle of the rats ankle was kept at $90^{\circ}$. At this angle the fused tetanic torque appeared to be maximal (Maenhout et al., 2000). The knee was kept at $90^{\circ}$.

\subsubsection{Exercise protocol}

A bipolar platinum hook electrode, inter-pole distance $0.8 \mathrm{~mm}$, was carefully attached to the peroneal nerve. A pulse generator (HSE 215/IZ, Freiburg, Germany) was used for electrical stimulation of the dorsal muscle complex. The stimulation voltage was determined from $150 \mathrm{~ms}$ contractions for maximal tetanic torque and ranged from 1 to $4 \mathrm{~V}$. The stimulation frequency was set at $71 \mathrm{~Hz}$, train duration at $1.1 \mathrm{~s}$ and pulse duration at $0.5 \mathrm{~ms}$. The resting period between contractions was $90 \mathrm{~s}$. This stimulation protocol was a compromise between torque modulation at low stimulation frequency $(<2 \%)$, fatigue (decrease of maximal torque during the protocol $<20 \%$ ) and minimum duration required for MR acquisition. Maximally 110 contractions were measured. 


\subsubsection{Imaging protocol}

The TA muscle was put in the center of the $4.7 T$ magnet (Varian, Palo Alto, CA), equipped with a high-performance gradient insert $(11 \mathrm{~cm}$ inner diameter, maximum gradient strength $220 \mathrm{mT} / \mathrm{m}$ ). The transverse and the longitudinal hind-leg axis approximately coincided with the $\mathrm{x}$ and $\mathrm{y}$-axis of the magnet respectively. A series of consecutive transverse gradient-echo multi-slice images of the hind-leg ( $T R=1 s$, $\mathrm{TE}=5 \mathrm{~ms}$, slice thickness $=1 \mathrm{~mm}$, FOV $=40 \times 40 \mathrm{~mm} ; 128 \times 128$ pixels) was measured. An orthogonal reference frame and a so called slice of observation (SOO) through the muscle were defined on the basis of anatomical landmarks in these images. This SOO was used as the reference slice (figure 5.2 ) because this slice has already been used in our research group for different purposes (van Donkelaar et al., 1999a; Gielen et al., 2000).
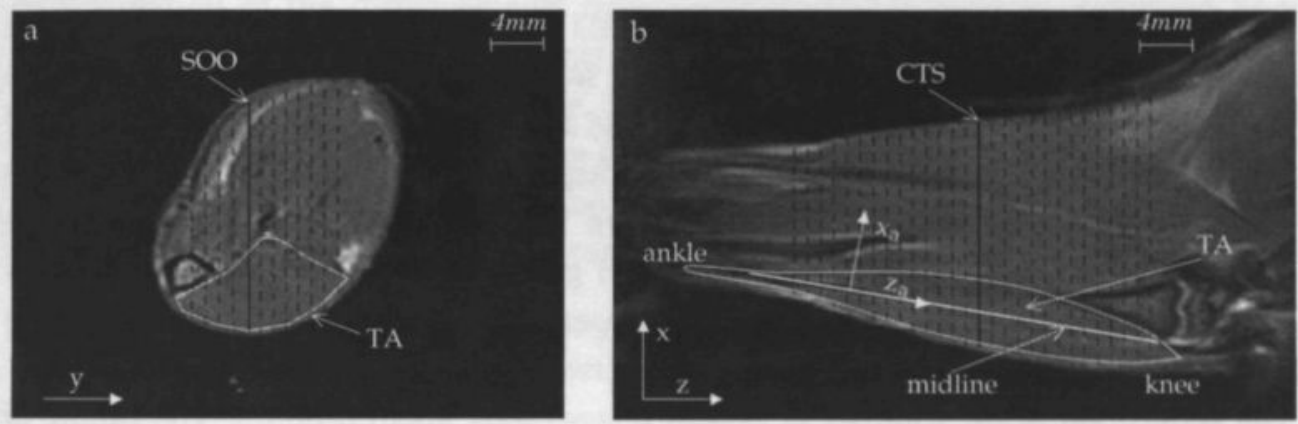

Figure 5.2: Localization of the acquired data set (rat 1) with definition of the orthogonal reference frame $(x, y, z)$ and the anatomical reference frame $\left(x_{a}, y, z_{a}\right)$. The contour of the TA is indicated by the white lines. a) Spin echo image of the central transverse slice (CTS) with projection of the orthogonal slice of observation (SOO, solid line) and adjacent sagittal slices (dashed lines). The $y$-direction coincides with the medial-lateral direction. b) Spin echo image of the sagittal slice of observation with projection of the CTS (solid line) and additional transverse slices (dashed lines) and the definition of the midline. The $\mathrm{x}$-direction coincides with the superficial-deep direction and the $z$-direction coincides with the longitudinal direction (Parameters spin echo multi-slice images: $T R=4 s, T E=20 \mathrm{~ms}$, slice thickness $=1 \mathrm{~mm}$, FOV $=40 \times 40 \mathrm{~mm} ; 128 \times 128$ pixels zero filled to $256 \times 256$ pixels).

To determine displacement during contraction in one direction, a 1D tagging grid was applied perpendicular to that direction by using spatial modulation of magnetization (SPAMM (Axel and Dougherty, 1989b,a)). The analysis technique required that the maximal displacements were not larger than half the grid line spacing in order to avoid aliasing errors. From the pilot experiments it was estimated that 
minimum line spacing of $3 \mathrm{~mm}$ for displacements in longitudinal direction and $4 \mathrm{~mm}$ for sagittal and transverse direction should be used. The following parameter settings were employed: $R F$ pulse $=B_{1}$-insensitive adiabatic segmented BIR 4 pulse according to Hendrich (1994), G(3mm line spacing) $=0.721 \mathrm{mT} / \mathrm{m}, \mathrm{G}(4 \mathrm{~mm}$ line spacing $)=0.541 \mathrm{mT} / \mathrm{m}$, effective $\mathrm{G}$ time $=11 \mathrm{~ms}$, tagging angle $\left.=0^{\circ}\left(90^{\circ}+180^{\circ}+90^{\circ}\right)\right)$ The FLASH sequence was used to image the tagging grids (parameter settings: $\mathrm{TR}=15.6 \mathrm{~ms}, \mathrm{TE}=7 \mathrm{~ms}$, flip angle $=8^{\circ}$, slice thickness $=1 \mathrm{~mm}$, field of view $=40 \times 40 \mathrm{~mm}$, number of pixels $=128 \times 128$ ). The read-out direction was chosen orthogonal to the tagging direction. In that case the frequency peaks in which the displacement information is stored are in the center of the k-space allowing reduced centric k-space sampling (McVeigh and Atalar, 1992; Fischer et al., 1994) to minimize the imaging time to $1 s$ (number of phase encoding steps $=64$ ).

Figure 5.2 shows how complete data sets of adjacent sagittal and transverse $1 \mathrm{~mm}$ slices were acquired. In order to reconstruct 3D displacements vertical (displacement in z-direction) and horizontal (displacement in $\mathrm{x}$-direction) tagging grid lines were applied to each sagittal slice. In the transverse slices grid lines parallel to the sagittal slices were applied in order to determine the displacement in y-direction. The average number of images required to cover the entire TA muscle was 55. To improve image quality, 2 scans were averaged per slice and grid line direction. Thus the TA was stimulated circa 110 times for 1.1s. Pilot experiments showed that muscle strength was preserved when a resting period of 90 s between successive contractions was used. The acquisition of the total set of tagged images took about 3 hours. The timing of experiment per slice and grid line direction is schematically depicted in figure 5.3. The entire experiment per rat including preparation and SOO selection took about 6 hours.

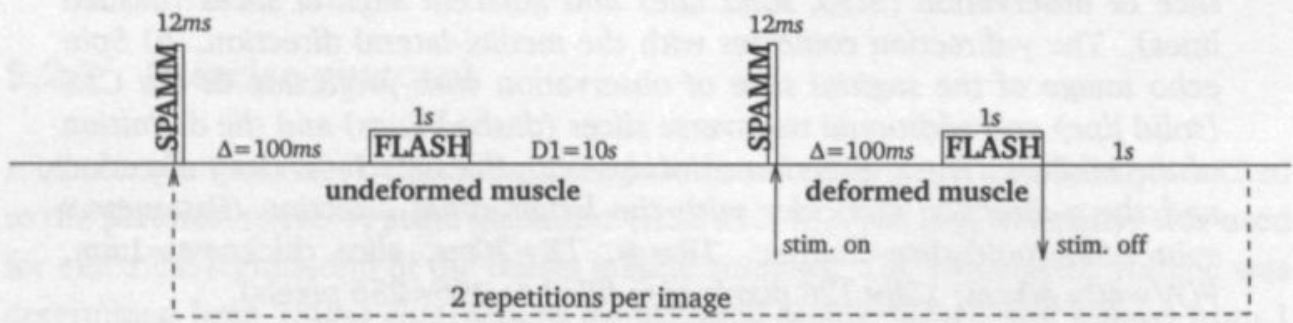

Figure 5.3: Timing of the tagging experiment. The delay time D1 allowed full recovery of the longitudinal magnetization, the delay time $\Delta$ was necessary for the muscle deformation to reach its steady state. Note: time axis not to scale. 


\subsubsection{Three dimensional displacements}

The applied method to quantify tag motion is related to methods to determine echo shifts in ultrasound pulsed Doppler instruments (Jong et al., 1990) and in phasebased optical flow (Jahne, 1993). The analysis method involved filtering of the stripe images of the TA muscle by multiplying the region around the first harmonic of the 2D-Fourier transform, corresponding to the stripes, by a 2D-Hanning window having a bandwidth of 0.8 times the grid frequency. From the filtered undeformed and deformed stripe images displacement maps were derived using one dimensional signal correlation. All analysis programs were written in Matlab 6.0.0.88 (The MathWorks Inc., Natick, Massachusetts).

Displacements of the pixels in $\mathrm{x}, \mathrm{y}$ and $\mathrm{z}$ direction were determined separately. As can be seen in figure 5.4 (in a 2D situation for the sake of easier visualization), determination of the displacements from undeformed tag lines to deformed lines results in an error in the displacements when the tag lines do not remain orthogonal and/or straight in the deformed state. Reconstruction of 3D displacements of voxels representing fixed volumes in the muscle tissue was done from deformed to the undeformed state. This introduces no errors in the displacements, because of the orthogonality of the grid lines in the undeformed state.

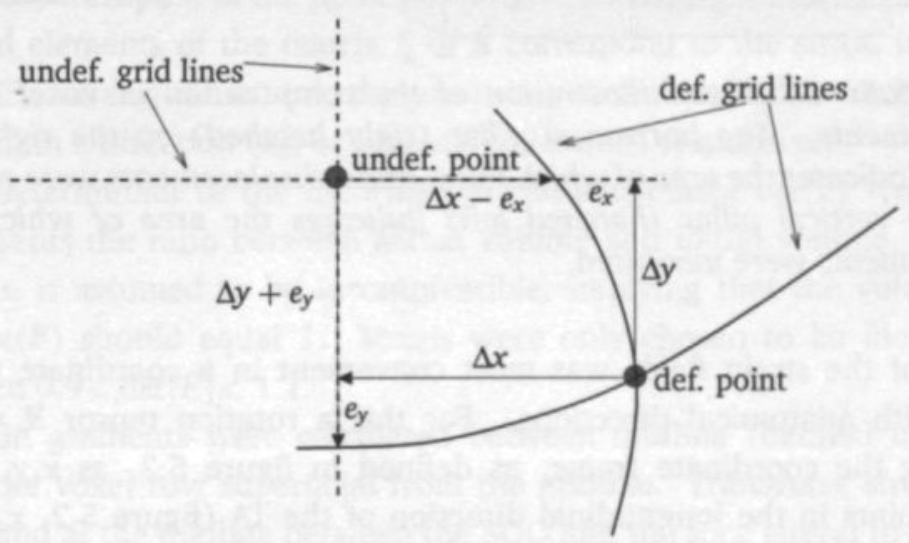

Figure 5.4: Artificial tag line in deformed (solid lines) and undeformed state (dashed lines) to illustrate the construction of $2 \mathrm{D}$ displacements of a material point. Displacement determination from undeformed grid lines to deformed grid lines $\left(\Delta x-e_{x}\right.$ and $\left.\Delta y+e_{y}\right)$ introduces an error $\left(e_{x}\right.$ and $\left.e_{y}\right)$ in both displacements.

For each tagged slice the region of the TA was selected on the basis of high resolution spin echo images (as shown in figure 5.2) of the same slice. From the sagittal slices, the pixel by pixel ( 1 pixel $\left.=0.31 \times 0.31 \mathrm{~mm}^{2}\right)$ displacements of the TA in $\mathrm{x}$ - 
and $\mathrm{z}$-direction were determined, while the $\mathrm{y}$-displacements were obtained from the transverse tagged images. Since the imaged slices had a thickness of $1 \mathrm{~mm}$, the reconstruction of the 3D displacements could not be carried out per voxel with the dimensions of the pixels $\left(0.31 \times 0.31 \times 0.31 \mathrm{~mm}^{3}\right)$. We chose to determine the $3 \mathrm{D}$ displacements per voxel of $1 \times 1 \times 1 \mathrm{~mm}^{3}$ based on the mean pixel displacements in that voxel. This choice seemed appropriate, because pixel displacements of a slice are the mean displacements of a volume of $0.31 \times 0.31 \times 1 \mathrm{~mm}^{3}$ (pixel $\times$ the slice thickness). The orientation of a voxel is schematically depicted in figure 5.5. Once the 3D displacement field of the deformed voxels was determined 3D interpolation was used to transform the displacements of the deformed voxels to displacements of the voxels in the undeformed state.

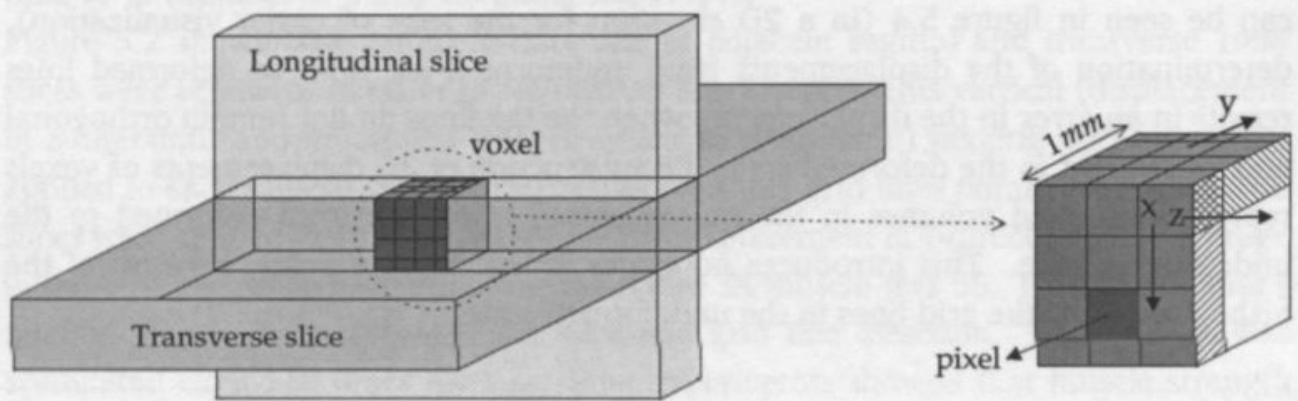

Figure 5.5: Schematic illustration of the computation of voxel by voxel displacements. The horizontal pillar (right hatched) on the right of the picture indicates the area of which the $\mathrm{x}$ - and $\mathrm{z}$-displacements were measured and the vertical pillar (hatched left) indicates the area of which the $y$ displacements were measured.

Evaluation of the strain fields was most convenient in a coordinate frame which coincides with anatomical directions. For this a rotation tensor $\boldsymbol{R}$ was defined, transforming the coordinate frame, as defined in figure 5.2, as $x, y, z$, such that the $\mathrm{z}$-axis points in the longitudinal direction of the TA (figure 5.2, $x_{a}, y, z_{a}$ ). This longitudinal direction corresponds approximately to the muscle fiber direction. The direction of $y$-axis remained the same. This reference frame will be referred to as the anatomical reference frame. The transformation of the displacements in the original frame to the displacements in the anatomical reference frame can be written as:

$$
\vec{u}_{a}=\boldsymbol{R}^{T} \cdot \vec{u}
$$

where $\vec{u}$ and $\vec{u}_{a}$ are the displacement vectors in the original and the anatomical reference frame respectively. 
The deformation-gradient tensor $\boldsymbol{F}$ of each voxel was calculated from the displacements of the voxel and the surrounding $3 \times 3 \times 3-1=26$ voxels by a second order approximation method (Geers et al., 1996). The tensor $\boldsymbol{F}$ is commonly used in the continuum mechanics to relate an undeformed infinitesimal material vector $d \vec{x}_{0}$ to its deformed state $d \vec{x}$ :

$$
d \vec{x}=\boldsymbol{F} \cdot d \vec{x}_{0}
$$

3D Green-Lagrange strains were computed from the deformation-gradient tensors. The Green-Lagrange strain is a frequently used measure in the continuum mechanics for length changes of material vectors and is defined as:

$$
\boldsymbol{E}=\frac{1}{2}\left(\boldsymbol{F}^{T} \cdot \boldsymbol{F}-\boldsymbol{I}\right)
$$

where $\boldsymbol{F}^{T}$ is the transpose of the deformation-gradient tensor and $I$ is the unit tensor. The diagonal elements of the matrix $\underline{E}$ of $\boldsymbol{E}$ correspond to the strain in anatomical $\mathrm{x}$-direction ( $E_{x x}$ or sagittal strain), the strain in y-direction ( $E_{y y}$ or transverse strain) and the strain in z-direction ( $E_{z z}$ or longitudinal strain) respectively.

Finally the determinant of the deformation-gradient tensor $\operatorname{det}(\boldsymbol{F})$ was calculated, which represents the ratio between actual volume and initial volume. Because the muscle tissue is assumed to be incompressible, implying that the volume remains constant, $\operatorname{det}(\boldsymbol{F})$ should equal 1 . Voxels were only chosen to be included in the analysis when $0.9<\operatorname{det}(\boldsymbol{F})<1.1$.

Sagittal strain gradients were calculated between midline (defined in figure 5.2) voxels and the voxel row superficial from the midline. Transverse strain gradients were calculated at the midline between the SOO and the slice lateral to it.

\subsubsection{Error analysis}

Errors in the acquired displacement field can arise from 1) the limitation in distinguishing displacement gradient and 2) displacement noise. Furthermore, the Green-Lagrange strains derived from the displacements were also perturbed by errors. Displacement gradients had to be described by limited frequencies defined by the bandwidth used to filter the stripe images. Therefore the spatial resolution, defined as the distance over which a gradient is smeared, for displacement inhomogeneities 
was limited. To test this resolution the analysis method was applied to artificial undeformed and deformed stripe images of $128 \times 128$ pixels. A line spacing of 12.8 pixels was chosen, corresponding to the maximum line spacing of $4 \mathrm{~mm}$ in the tagged images of the TA. The artificial deformed stripe image involved strong gradients occurring over a distance of one pixel.

The displacement noise was tested by determining the standard deviation of the mean displacement acquired by the analysis method from two separate tagged MR images with a line spacing of $4 \mathrm{~mm}$ of an undeformed TA muscle.

To analyze the errors with respect to the $3 \mathrm{D}$ strains the second order approximation method was tested by using a known artificial 3D displacement field. The regular particle distribution consisted of a $11 \times 11 \times 3$ particle field which is similar to the displacement fields of three sagittal slices of the TA which were used to compute the strain field of the central slice of these three slices. The displacement field was such that a quadratic $E_{z z}$ strain field and a constant $E_{y y}$ strain field $\left(E_{x x}=0\right)$ were adopted. The maximal $E_{z z}$ strain was 0.33 and $E_{z z}$ was 0.105 . The artificial displacement field was perturbed by a normally distributed random noise signal with a standard deviation which was also observed in the displacement field acquired from two separate tagged MR images of an undeformed TA muscle.

\subsubsection{Statistics}

In the following sections all data are given in mean \pm standard deviation. 


\subsection{Results}

The muscle torque during the first contraction was $21.3 \pm 5.1 \mathrm{Nmm}$. The muscle strength remained above $80 \%$ of the initial strength during the protocol.

Figure 5.6 shows the raw data of one rat. The top images show the tagged MR images acquired from the TA muscle at rest (indicated by the white lines), while the bottom images show the deformed muscle at steady-state contraction. The raw data in figure 5.6b show that the superficial (skin) z-displacements were small compared to the deformation of the deeper muscle tissue. In the transverse direction (figure 5.6c) the skin did move with the muscle tissue. In the sagittal slice, the dorsal muscle complex deforms only slightly as was expected for a non-stimulated muscle. In the transverse slice much more deformation was visible in the dorsal muscle complex, probably caused by passive deformation.

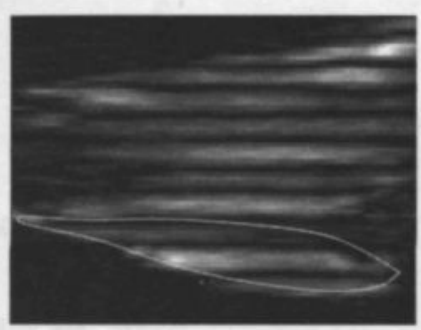

a

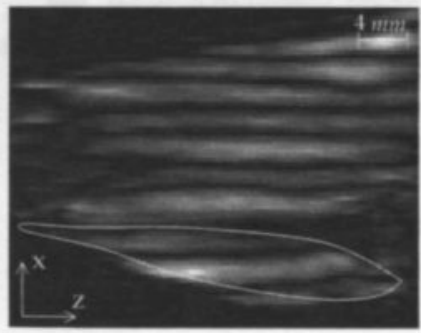

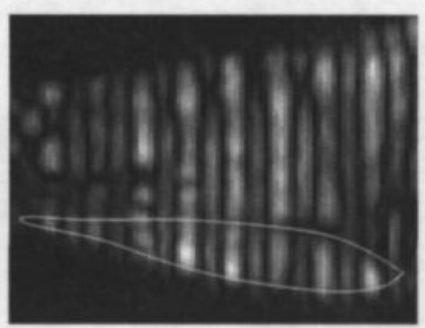

b

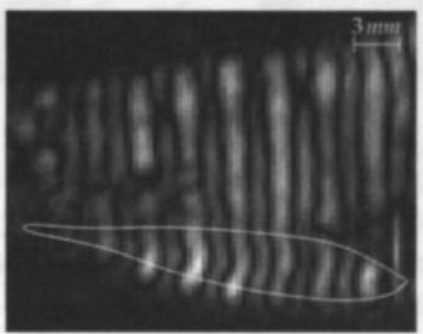

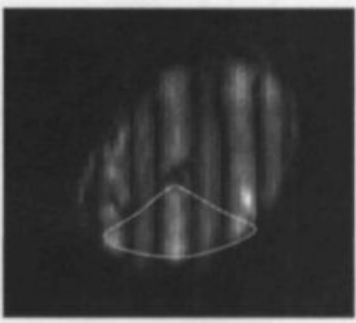

c

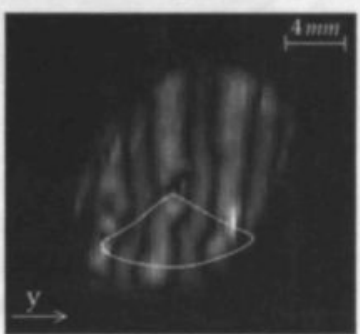

Figure 5.6: Tagged MR images (rat1), acquired from muscle at rest (top) and from muscle at steady-state contraction (bottom). The position of the undeformed TA and the distal tendon is indicated by the white lines. a) Sagittal slice horizontal lines. b) Sagittal slice vertical lines. c) Transverse slice, vertical lines.

The mean fraction of voxels not included in the analysis because $0.9<\operatorname{det}(\boldsymbol{F})<1.1$ was $67 \%$. 
Figure 5.7 shows a typical strain field of the $\mathrm{SOO}$ of one rat (rat 1). The stain field was highly inhomogeneous in both sagittal and longitudinal direction. The following figures illustrate these inhomogeneities more clearly.
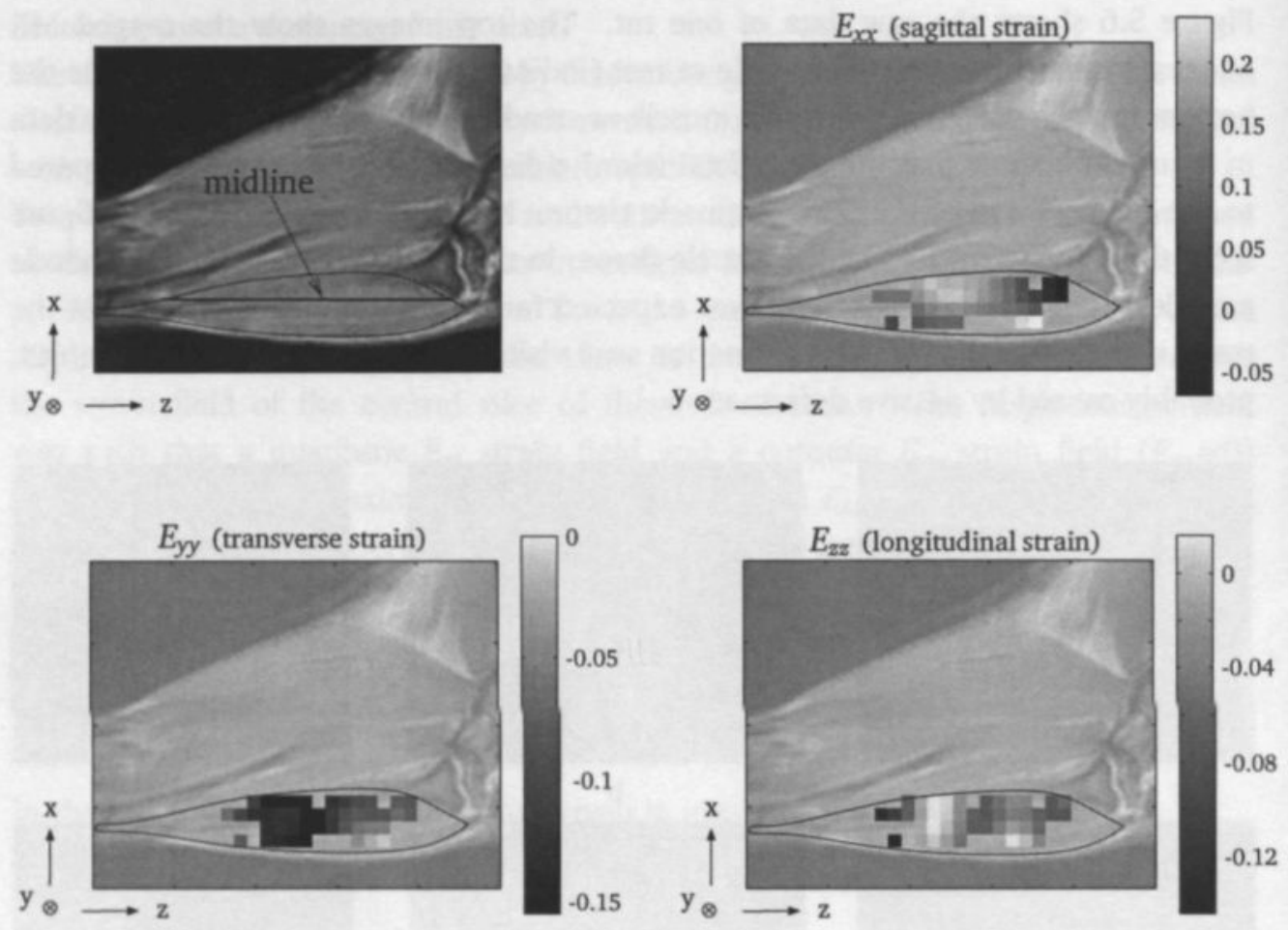

Figure 5.7: Green-Lagrange strain in the $S O O$ of rat 1, with the definition of the midline. The $x$-direction coincides with the superficial-deep direction, the $y$-direction with the medial-lateral direction and the $z$-direction with the distal-proximal direction. 
In figure 5.8 the strains of the midline of six consecutive sagittal planes of rat 1 are shown. The transverse distribution of the strains was also inhomogeneous. The sagittal strain was more positive medially than laterally. The strain as a function of the longitudinal position however seemed comparable. Strains differed most at a scaled longitudinal position of 0.6. This difference equaled about 0.2 . The transverse strains showed comparable behavior, but mirrored: medially more negative strains occurred. The longitudinal strains showed more variation in the longitudinal than in the transverse direction. Distal strains were about -0.1 , while the smallest strains occurred at a scaled position of 0.4 and were about 0 . The maximal difference in the transverse direction was about 0.05 .

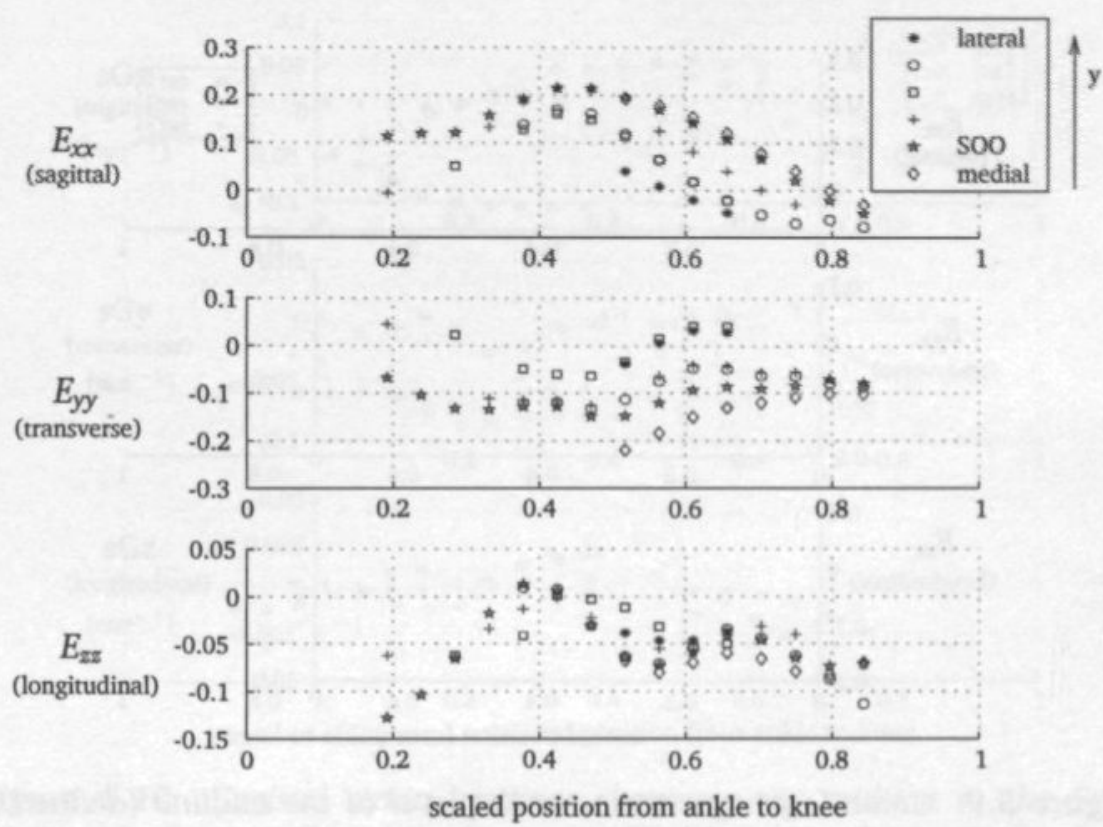

Figure 5.8: Green-Lagrange strain at the level of the midline (defined in figure 5.7) as a function of the scaled longitudinal position of six consecutive sagittal planes of rat 1 with an inter-distance of $1 \mathrm{~mm}$. 
Figure 5.9 shows the strains in the SOO in the midline of the TA of all three rats. The sagittal strain did not show a consistent picture. The most prominent difference is that the sagittal strain of rat 2 in the middle of the TA was negative, while it was positive for the other rats. The proximal sagittal strains also showed obvious differences ( 0.2 for rat 3 vs. -0.05 for rat 1$)$. Transverse and longitudinal strain showed less variation between rats. Transverse strain was most negative at a scaled position between 0.3 and 0.5 , and increases distally and proximally. At the position of 0.4 all longitudinal strains were about 0 and they decreased in distal direction. They decreased to approximately -0.07 in proximal direction to a scaled position of about 0.55 .
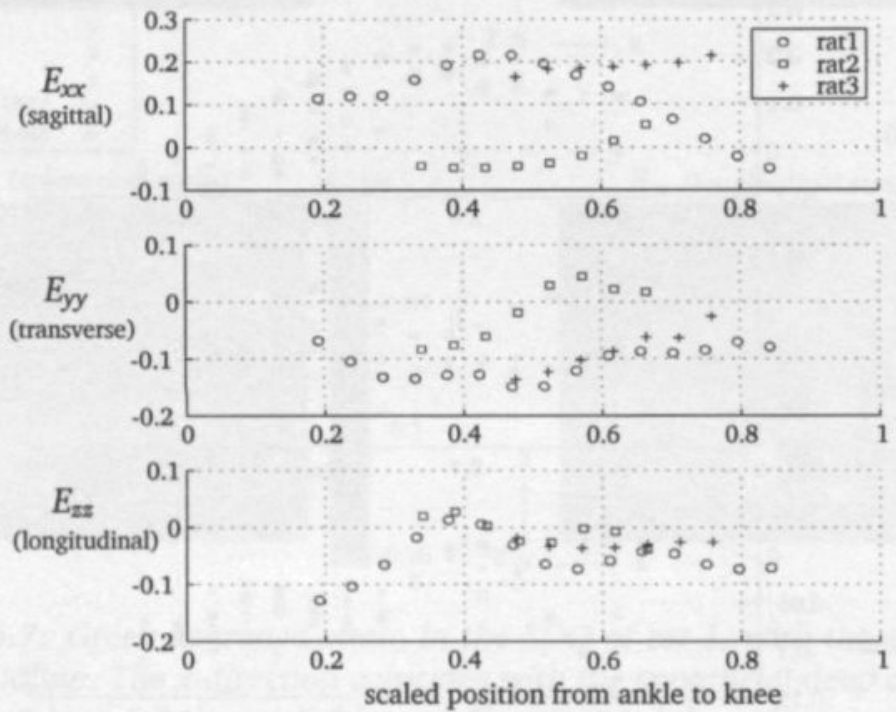

Figure 5.9: Green-Lagrange strains at the level of the midline (defined in figure 5.7) as a function of the scaled longitudinal position. $E_{x x}$ represents sagittal strain, $E_{y y}$ transverse strain and $E_{z x}$ represents longitudinal strain. 
The sagittal strain gradients are shown in figure 5.10. Proximally, the gradients of the sagittal strain were negative, indicating that deep strains were less positive than superficial. Distally the gradients were positive. The gradients of the transverse strain were about zero in the distal region and decreased in proximal direction. This implies that in the proximal area of the SOO the deep transverse strains were more negative than the superficial ones. The gradients of the longitudinal strains were between $-0.025 \mathrm{~mm}^{-1}$ and $0.025 \mathrm{~mm}^{-1}$, which was small compared to the gradients of the sagittal and transverse strains. Generally the proximal gradients were negative for all rats. The deep strain in the proximal part was thus slightly more negative than the superficial strain.
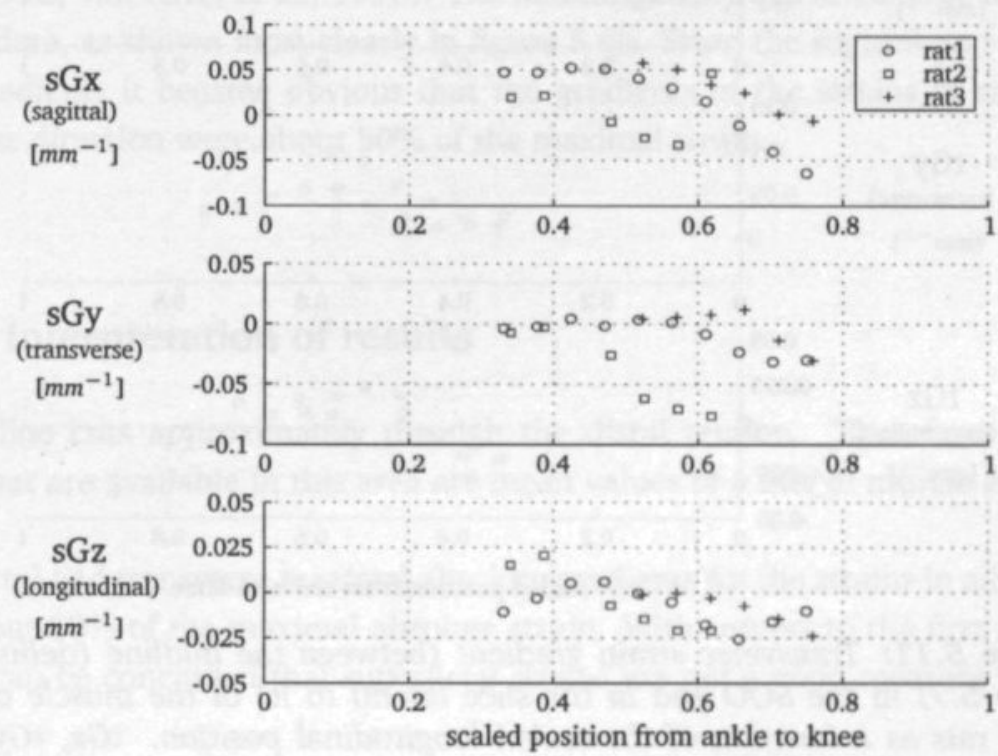

Figure 5.10: Sagittal strain gradient (between the midline in the SOO (defined in figure 5.7) and the line superficial from it) of the muscle of the three rats as a function of the scaled longitudinal position. $s G x, s G y$ and $s G z$ represent the gradient of the sagittal, transverse and longitudinal strains respectively. 
Figure 5.11 shows the transverse strain gradients. Generally, the gradients of the sagittal strains were negative. This means that medially the sagittal strains were more positive than laterally. All gradients of the transverse strain were positive. The gradients of the longitudinal strains were small compared to the gradients of the sagittal and transverse strains. The transverse gradients were between $-0.025 \mathrm{~mm}^{-1}$ and $0.025 \mathrm{~mm}^{-1}$. Generally the proximal gradients were positive, while the distal gradients were negative.
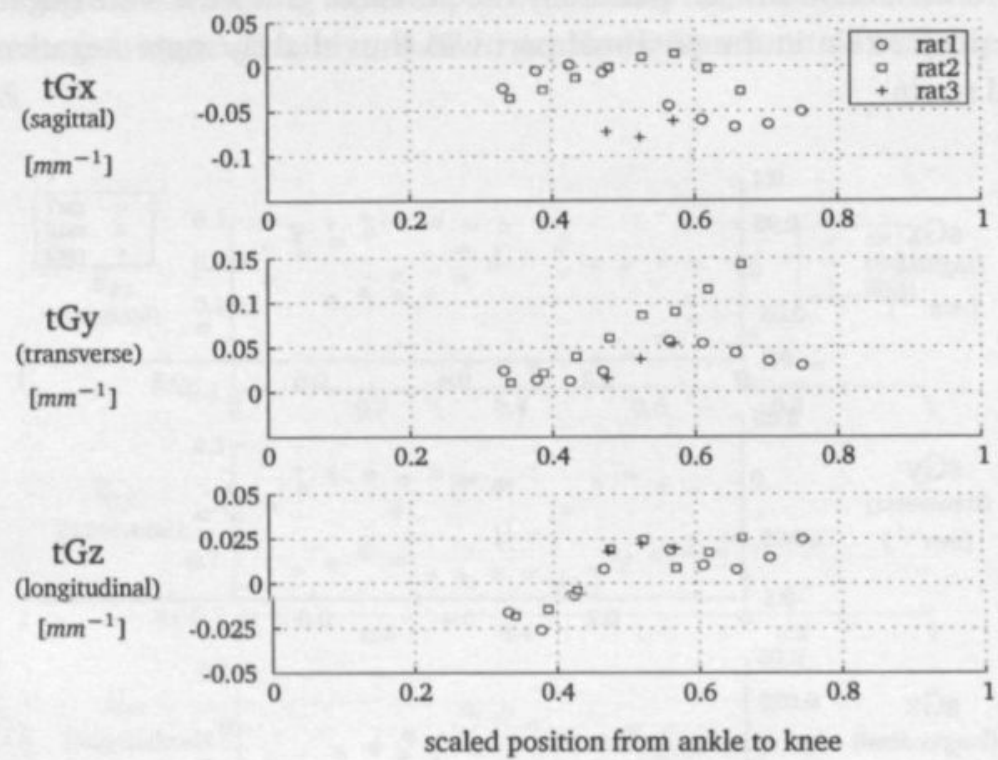

Figure 5.11: Transverse strain gradient (between the midline (defined in figure 5.7) in the $S O O$ and in the slice lateral to it) of the muscle of the three rats as a function of the scaled longitudinal position. $t G x, t G y$ and $t G z$ represent the gradient of the sagittal, transverse and longitudinal strain respectively.

\subsubsection{Error analysis}

From artificial undeformed and deformed stripe images it was determined that the spatial resolution for displacement gradients, defined as the distance over which a gradient is smeared, was 5 pixels or $1.56 \mathrm{~mm}$.

The determined displacement fields were perturbed by a noise signal with a standard deviation of $0.1 \mathrm{~mm}$.

The maximum errors in the derived 3D strains determined from a known artificial 3D displacement field perturbed by a normally distributed random noise signal with a standard deviation of $0.1 \mathrm{~mm}$ were 0.01 in the midfield and 0.05 at the edges. 


\subsection{Discussion}

\subsubsection{General}

This paper presents non-invasively acquired in vivo 3D voxel by voxel strain maps of the whole tibialis anterior muscle of rat during steady-state isometric contraction. To the best of the authors knowledge this is the first time that 3D-strain fields of a contracting skeletal muscle are reported. The internal 3D strain distribution in a contracting muscle appeared highly inhomogeneous. The observed inhomogeneity agrees with observations on superficially measured deformations (van Donkelaar et al., 1999b; van Bavel et al., 1996). The inhomogeneity can already be observed in the raw data, as shown most clearly in figure $5.6 \mathrm{~b}$. From the sagittal and transverse strain gradients it became obvious that the gradients of the strains in sagittal and transverse direction were about $50 \%$ of the maximal strain.

\subsubsection{Interpretation of results}

The midline cuts approximately through the distal tendon. Therefore, the distal strains that are available in this area are mean values of a mix of muscle and tendon tissue.

The sagittal and transverse maximal absolute gradients for the strains in all directions were about $50 \%$ of the maximal absolute strain. With respect to the first aim of our study it can be concluded that superficial strains are not a good measure for deeper strains.

The suitability of sagittal plane models for the simulation of local mechanical quantities within the muscle can be questioned when considering the transverse strain gradients. Mean transverse muscle surface strain changes with muscle length were measured by van Donkelaar et al. (1999b), who already concluded that sagittal plane models assuming no deformation in the transverse direction can not be used to calculate the effect of muscle length changes on deformation during contraction. Here 3D strains of about $70 \%$ of the muscle volume, instead of superficial mean transverse strains, are presented, showing that the maximal absolute transverse strain gradients were in the order of $0.1 \mathrm{~mm}^{-1}$. This is of the same order as the maximal absolute sagittal gradient. This leads to the conclusion that deformation in the transverse direction is not negligible compared to the other two directions. When the aim of a muscle model is to simulate realistic 3D muscle deformation it can be concluded that models with a realistic 3D geometry will be needed. 


\subsubsection{Error analysis}

Large errors in the acquired strain field could result from e.g., changes in the displacement field during the imaging of the successive slices or voxel displacments which are the mean value of a mix of different tissues (e.g. muscle and tendon). The volume of the muscle should remain constant during contraction. A constant volume implies that $\operatorname{det}(\boldsymbol{F})$ equals 1 . Voxels, for which $0.9<\operatorname{det}(\boldsymbol{F})<1.1$, were not included in the analysis. The error in $\operatorname{det}(\boldsymbol{F})$ can be approximated by the sum of the errors of the Green-Lagrange strains in the three directions. The maximum errors in the derived strains equaled 0.01 in the midfield and 0.05 at the edges. The condition to trust the determined strains thus roughly coincided with the maximum error caused by strain calculation. With this approach, large errors in the acquired strain field are avoided.

Strain gradients may be underestimated since the spatial resolution for displacement gradients was $1.56 \mathrm{~mm}$. Large gradients can especially exist at tissue boundaries like at the internal distal tendon or at the proximal aponeurosis.

From the fact that the differences between the rats in distal and proximal displacements (not shown) were less pronounced than the medial differences suggests that the fixation of the rats leg was reproducible.

\subsubsection{Experimental procedure}

The used displacement analysis technique has the advantage that it does not depend on the detection of separate tags, is insensitive to noise in images, and requires relatively little user interaction. A methodological prerequisite in the present study was that the maximal displacements were not larger than half the grid line spacing in order to avoid aliasing errors. These errors did not occur and from the measured minimal and maximal displacements (not shown) it could be seen that the $3 \mathrm{~mm}$ linespacing for $\mathrm{z}$-displacement and $4 \mathrm{~mm}$ for $\mathrm{x}$ - and $\mathrm{y}$-displacement was sufficiently large. The displacement of about $70 \%$ of the TA muscle tissue was successfully determined in a regular grid with inter-distances of $1 \mathrm{~mm}$. Due to the small area of TA in the transverse images, especially y displacements of the distal part of the TA could not be determined.

If frontal slices with tag lines parallel to the SOO are used for determining y displacement, instead of transverse slices, less images and consequently less contractions are needed. This would make it possible to sample displacements in a larger part of the TA. 


\subsubsection{Future research}

Maximally 110 contractions were measured during the protocol, with torque deficits less than $80 \%$. If it is assumed that these torque deficits do not significantly change the strain distributions, a series of contractions may be used in the future to determine the time course of the internal muscle deformation of a restricted area in the same way as was done in the field of cardiac deformation studies (Kuijer et al., 2000; Thomas and McVeigh, 1997; Alistair and Young, 1996)

The observed 3D distribution of displacements and strains points towards the need for 3D muscle models to study the mechanical muscle behavior during contraction. Existing 2D numerical models (Gielen et al., 2000; MotabarZadeh, 1998; Vankan et al., 1998, 1997) can be extended to realistic 3D geometries in order to realistically simulate mechanical muscle behavior. The experimental data presented in this paper can be used to validate the newly developed 3D models. Parametric studies of the models may help to gain insight in the observed variation in the local deformation of the muscle tissue.

\section{Acknowledgment}

We gratefully acknowledge the excellent technical assistance of G. van Vliet during the MRI studies. Furthermore the authors are grateful to M. Kruiskamp, L. Geerts, and R. Kerckhoffs for their preliminary investigations. 
64

Chapter 5 


\section{Chapter 6}

\section{Simulated 3D displacement and strain fields within}

isometrically contracting muscle

\subsection{Introduction}

Muscle adaptation and damage are initially local phenomena at the level of individual cells (Fitts et al., 1991; van der Meulen et al., 1993; Lieber and Fridén, 1993; Lieber et al., 1994; Koh and Herzog, 1998a). To study how adaptation and damage are invoked knowledge of local muscle loading is indispensable. Numerical muscle models have been developed to study the local muscle loading (Gielen et al., 2000; Vankan et al., 1998; van der Linden et al., 1998b; van Leeuwen and Spoor, 1996). So far these models were limited to 2D sagittal plane geometries.

From Magnetic Resonance (MR) images with tissue tagging of an isometrically contracting tibialis anterior (TA) muscle of a rat $(n=3), 3 D$ displacement fields and Green-Lagrange strains were derived at the plateau phase of an isometric contraction by Maenhout et al. (2001a) (chapter 5). It appeared that deformation in the transverse direction was not negligible compared to the other two directions. This was also shown by van Donkelaar et al. (1999b). Therefore it was concluded that to simulate realistic 3D muscle deformation, sagittal plane models should be extended to models with a realistic 3D geometry.

The purpose of the present study is 1) to extend the 2D sagittal plane continuum model of Gielen et al. (2000) to a model with a more realistic 3D geometry, 2) 
to evaluate the effect of using a $2 \mathrm{D}$ or $3 \mathrm{D}$ geometry on the computed strain and displacement fields during contraction, 3 ) to study the effect of the sarcomere length distribution on the strain and displacement fields and 4) to make a first step towards model validation. The finite element method was used to solve the conservation laws of the 3D continuum model. A new solid element was developed to solve the field equations (van Oijen et al., 2001), using a mixed, updated Lagrange formulation instead of a total Lagrange approach Gielen et al. (2000). This element improved the robustness of the computations and the ability to deal with incompressible behavior.

\subsection{Methods}

This section describes a continuum model of the rat TA muscle and the experiments and simulations that were carried out to acquire displacement and strain fields during the plateau phase of isometric contractions. In this chapter isometric contractions are defined as contractions during which the total length of the muscle-tendon complex remains constant.

\subsubsection{Model equations and parameters}

In the continuum approach the local stress $\sigma$ in the tissue is defined as a superposition of the active stress $\sigma_{a} \vec{e}_{f} \vec{e}_{f}$ and the passive stress $\sigma_{p}$ caused by the deformation of the tissue:

$$
\sigma=\sigma_{a} \vec{e}_{f} \vec{e}_{f}+\sigma_{p}
$$

where $\vec{e}_{f}$ denotes the local averaged fiber direction. The muscle obeys the balance of momentum. When ignoring body forces and inertial forces this can be given as:

$$
\vec{\nabla} \cdot \sigma^{c}=\overrightarrow{0}
$$

The incompressibility constraint is expressed as

$$
J=\operatorname{det}(\boldsymbol{F})=1
$$

where $\boldsymbol{F}$ represents the deformation-gradient tensor.

The constitutive behavior of the contractile myofibers is described by a distributionmoments (DM) approximated two-state Huxley cross-bridge model (Zahalak and Ma, 1990; Zahalak, 1996). The parameters in this model have a physical meaning and some can be measured directly. The Huxley model and the DM theory are discussed 
in detail in Zahalak (1981, 1986); Zahalak and Ma (1990); Ma and Zahalak (1991, 1987). Therefore only a short summary will be given in this chapter. The basic Huxley theory focuses on an ensemble of myosin heads which are assumed to be capable of binding to an actin binding site to form a so called cross-bridge. During contraction a fraction of all cross-bridges is attached. Every attached cross-bridge has its own dimensionless attachment length $\xi$. The distribution of attached cross-bridges with respect to their length is given by the function $n(\xi, t)$ and the rate of change of this distribution can be expressed with a modified two state Huxley equation:

$$
\frac{d n(\xi, t)}{d t}=\frac{\partial n(\xi, t)}{\partial t}-u(t) \frac{\partial n(\xi, t)}{\partial \xi}=r(t) f(\xi)\left[\alpha\left(l_{s}\right)-n(\xi, t)\right]-g(\xi) n(\xi, t)
$$

where $u(t)$ is the scaled shortening velocity of a half sarcomere. The velocity is set to zero while it was shown by Gielen (1998) that it does not effect the steady state solution during the plateau phase. The activation factor $r(t)$ depends on the calcium present in the muscle fiber and $\alpha$ is the overlap factor of the filaments. The parameters $f(\xi)$ and $g(\xi)$ represent the rate parameters for attachment and detachment respectively of myosin to actin and are postulated to vary with the bond length $\xi$, as shown in figure 6.1 .

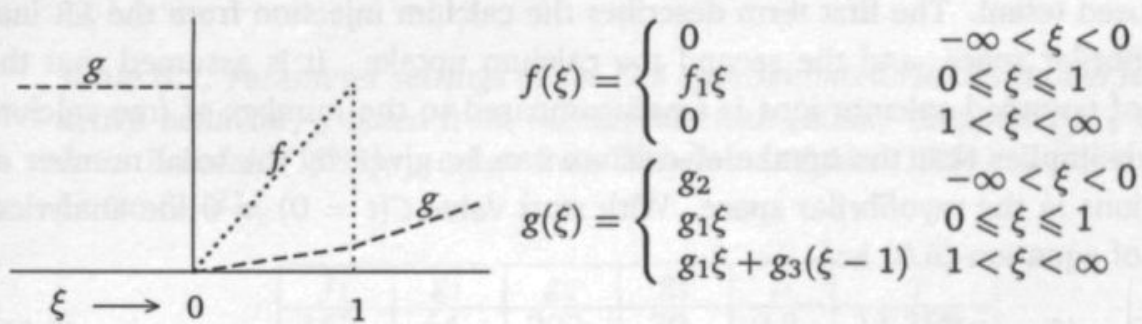

Figure 6.1: Rate constants for attachment $(f)$ and detachment $(g)$ of myosin to actin. $\xi=1$ is the maximum displacement of the myosin head at which binding can occur.

The overlap factor $\alpha$ is described as (Herzog, 1992)

$$
\alpha(l)=\left\{\begin{array}{ll}
1-6.25(l-1)^{2} & l \leq 1 \\
1-1.25(l-1) & l>1
\end{array} \quad l=l_{s} / l_{s, o p t}\right.
$$

where $l_{s}$ and $l_{s, o p t}$ are the current and the optimal sarcomere length respectively. The activation factor $r(t)$ describes the calcium activation in the Huxley cross-bridge model. A myosin head can only interact with an actin binding site if two calcium ions 
are bound to the specific receptor sites on the troponin molecule of that actin site. The fraction activated actin is defined as $r(t)$ and depends on the free calcium present within the muscle (Zahalak and Ma, 1990, 1997):

$$
r(t)=\frac{C^{2}}{C^{2}+\mu C+\mu^{2}}
$$

with $C$ representing the ratio of the free calcium concentration in the myofibrilar space with respect to the maximum myofibrilar calcium concentration and $\mu$ the troponincalcium reaction ratio constant. The calcium concentration is regulated by the electric stimulation causing the release of calcium from the sarcoplasmatic reticulum (SR). Instead of the more complex model of this process of Zahalak and Ma (1990), the simpler representation of Hatze (1981) was used, since this model gives comparable results for supra-maximal contractions. The representation of Hatze (1981) is written as:

$$
\partial C / \partial t=\gamma(\nu-C)
$$

where $\gamma$ represents a fiber-type dependent rate parameter and $\nu$ the stimulation frequency with respect to the fused tetanic stimulation frequency. Here $\nu$ is set to one while the experimental data to which simulations will be compared were acquired during fused tetani. The first term describes the calcium injection from the SR into the myofibrilar space, and the second the calcium uptake. It is assumed that the number of bounded calcium ions is small compared to the number of free calcium ions. This implies that the uptake of calcium can be given by the total number of calcium ions in the myofibrilar space. With start value $C(t=0)=0$ the analytical solution of equation (6.9) is:

$$
C(t)=\left(1-e^{-\gamma t}\right)
$$

Substituting equation (6.10) into (6.8) gives an analytical expression of the activation factor $r(t)$.

The active muscle stress can be determined from the distribution of attached crossbridges $n$. In the Huxley theory it is assumed that the cross-bridge force depends linearly on the attachment length $\xi$, with constant $\kappa$. The active Cauchy stress $\sigma_{a}$ generated by all cross-bridges in a slice of half sarcomeres can be shown to be (Zahalak and Ma, 1990):

$$
\sigma_{a}(t)=c_{a} \lambda Q_{1}(t)
$$

where $Q_{1}$ is the first moment of function $n(\xi, t), c_{a}$ is a material constant and $\lambda$ is the extension ratio in fiber direction. In general the $k$-th moment of a distribution function $n$ is defined as: 


$$
Q_{k}(t)=\int_{-\infty}^{\infty} \xi^{k} n(\xi, t) d \xi
$$

As can be seen from equation (6.11), the active stress $\sigma_{a}$ does not depend on the exact shape of the function $n$, but on the first moment of the function $n$. By assuming a Gaussian distribution for the function $n$, Zahalak (1981) approximated the partial differential equation (6.4) by the following set of ordinary first order differential equations:

$$
\dot{Q}_{k}=\alpha(E) r(C) \beta_{k}-r(C) \phi_{1 k}\left(Q_{0}, Q_{1}, Q_{2}\right)-\phi_{2 k}\left(Q_{0}, Q_{1}, Q_{2}\right)-k u(\dot{E}) Q_{k-1}, \quad k=0,1,2
$$

where the superimposed dot denotes time differentiation and the functions $\beta_{k}, \phi_{1 k}$ and $\phi_{2 k}$ have been defined in Zahalak (1981). The factor $\alpha$ depends on the strain $\boldsymbol{E}$ and it should be reminded that the last velocity term in equation (6.13) was set to zero.

The parameter values of the DM approximated Huxley model used in the continuum model are listed in table 6.1.

Table 6.1: Parameter settings of the DM approximated Huxley model for the active behavior; $a$ taken from Maenhout et al. (2000) (chapter 3); $b$ taken from Zahalak (1981); c taken from Zahalak and Ma (1990); $d$ taken from Hatze (1981).

\begin{tabular}{|c|c|c|c|c|c|}
\hline$f_{1}$ & $g_{1}$ & $g_{2}$ & $g_{3}$ & $\mu$ & $\gamma$ \\
\hline $163_{a}$ & $64_{a}$ & $200_{b}$ & $30_{b}$ & $0.2_{c}$ & $11.255_{d}$ \\
{$\left[s^{-1}\right]$} & {$\left[s^{-1}\right]$} & {$\left[s^{-1}\right]$} & {$\left[s^{-1}\right]$} & {$[-]$} & {$\left[s^{-1}\right]$} \\
\hline
\end{tabular}

The passive tissue stress for both the passive muscle tissue and the connective tissue (distal tendon and proximal aponeurosis) is described by an incompressible neoHookean model

$$
\sigma_{p}=-p I+\tau
$$

with $p$ the pressure, $I$ the unit tensor and $\tau$ the extra stress tensor described as

$$
\tau=G(B-I)
$$

with $\boldsymbol{B}$ the Finger tensor defined as $\boldsymbol{F} \cdot \boldsymbol{F}^{T}$. The material parameter $\mathrm{G}$ is the shear modulus. 
The finite element method was used to solve the conservation laws. In contrast to Gielen et al. (2000) an Updated Lagrange formulation (van Oijen et al., 2001) instead of a Total Lagrange approach is used to solve the equations. A new solid element was developed to solve the field equations (van Oijen et al., 2001). This element improved the robustness of the computations and the ability to deal with incompressible behavior. The improvement involved, 1) an updated Lagrange instead of a total Lagrange approach, 2) a mixed formulation instead of the natural penalty or enhanced stiffness method to meet the incompressibility constraint, 3) an isoparametric quadratic instead of linear brick elements. Isotropic material behavior was employed and is supported by the findings of Bosboom et al. (2001b), who showed that the difference between simulations of passive muscle loading with anisotropic or isotropic behavior were neglectable. Another important distinction compared to the model of Gielen et al. (2000) is the extension of the 2D sagittal plane geometry to a more realistic $3 \mathrm{D}$ geometry. For a detailed description of the numerical solution strategy see van Oijen et al. (2001).

\subsubsection{Model geometry of tibialis anterior muscle}

The muscle geometry was derived from normal high-resolution MR-images (figure 6.2a). The fiber direction was measured with Diffusion Tensor Imaging (DTI) data (van Doorn et al., 1996; van Donkelaar et al., 1999a) and were described by polynomial fits of the measured directions (Gielen, 1998). Using polynomials was preferable since the directions could be incorporated regardless the coarseness of the element division. This resulted in a geometry and fiber direction as shown in figure 6.2b (Gielen, 1998). The geometry was divided into iso-parametric quadratic elements as shown in figure $6.2 \mathrm{c}$. It should be noted that in this figure the grid coarseness of the nodes of the quadratic elements is shown. 
a

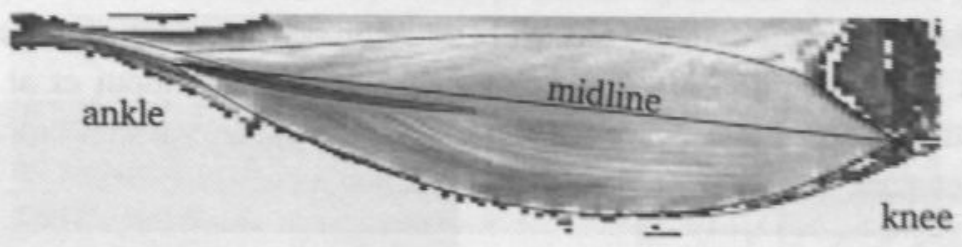

b
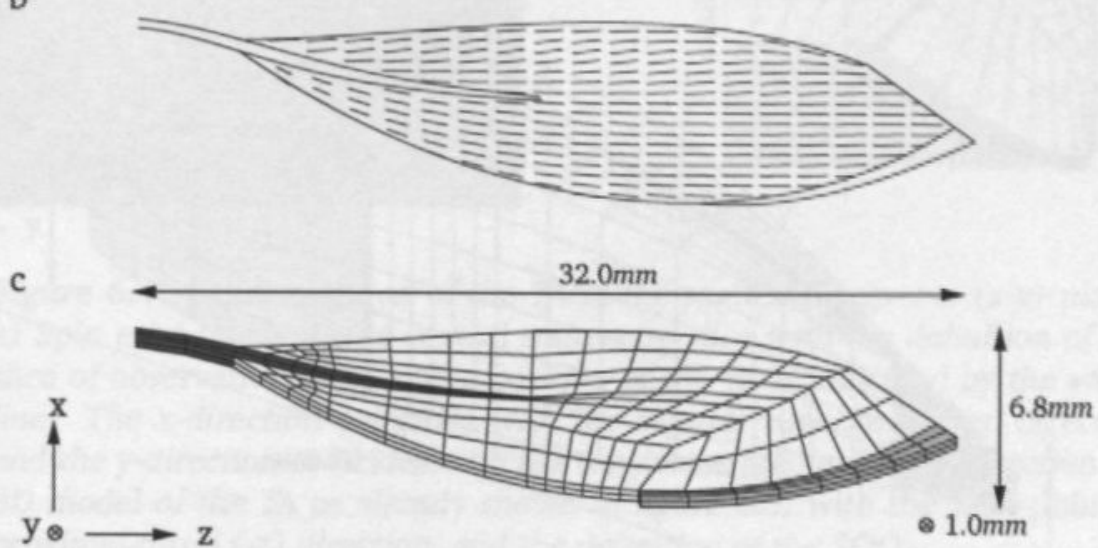

Figure 6.2: $2 D$ continuum model of the cross section of the mid-sagittal plane of the TA of the rat. a) Geometry of the model derived from a MR image and definition of the midline. b) Fiber directions. The fibers are parallel to the boundaries and have an angle with respect to the insertion sites. c) Grid coarseness of the nodes of the quadratic elements. The distal tendon, consisting of an external part and an internal part referred to as the distal aponeurosis, is colored dark gray and the proximal aponeurosis is colored light gray. The $x$-direction coincides with the sagittal superficial-deep direction, the $y$-direction with the transverse medial-lateral direction and the $\mathrm{z}$-direction with the longitudinal distal-proximal direction.

Although 3D elements were used for the geometry of Gielen et al. (2000) the model will be referred to as a $2 \mathrm{D}$ model since it represents a flat slice within the TA with a thickness of $1 \mathrm{~mm}$ (one element, having three nodes in transverse direction). For the 3D model three elements (seven nodes in the transverse direction) instead of one were used in the transverse direction. The side of the model representing the medial side was chosen to be flat, while the side representing the lateral side was given a curvature as shown in figure 6.3. This choice was based on visual inspection of a free dissected TA, showing an asymmetry in the transverse direction such that the tendon and knee are fixed in the medial direction. The fiber directions in the sagittallongitudinal plane were the same as those of the 2D model, while the direction of 
the fibers in the transverse direction are parallel to the curvature in this direction. The dimensions, as given in figure 6.3, were determined from a set of transverse and longitudinal high-resolution MR-images of a TA muscle (Maenhout et al., 2001a). Again it should be noted that figure 6.3 shows the grid coarseness of the nodes of the quadratic elements.

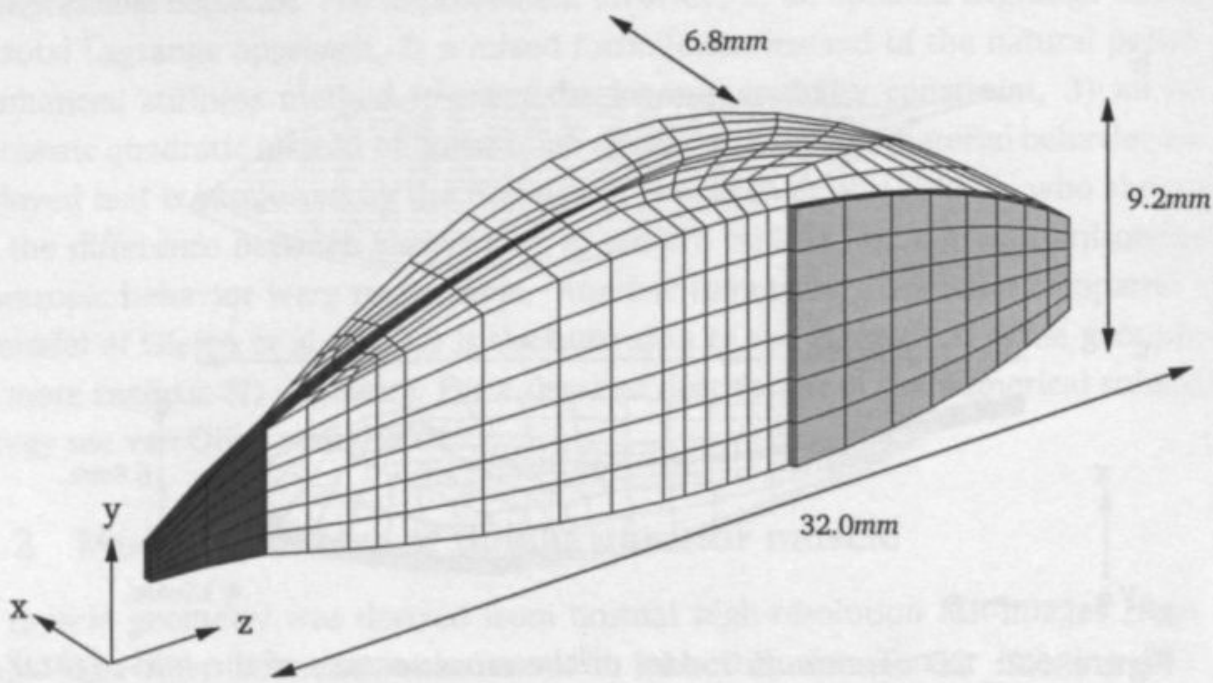

Figure 6.3: $3 \mathrm{D}$ model of the TA of the rat, indicating the grid coarseness of the nodes of the quadratic elements. The distal tendon, consisting of an external part and an internal part referred to as the distal aponeurosis, is colored dark gray and the proximal aponeurosis is colored light gray. The $x$-direction coincides with the sagittal superficial-deep direction, the $y$ direction with the transverse medial-lateral direction and the z-direction with the longitudinal distal-proximal direction.

In order to interpret the simulation results and compare them with the MRI studies, the midline of the model is defined in a similar way as for the MRI experiments (Maenhout et al., 2001a). The orientation of the midline is shown in figure 6.2a. For the 3D model the midline cuts through the TA geometry at $y=3 \mathrm{~mm}$ based on the definition of the midline in the slice of observation (SOO) as defined in Maenhout et al. (2001a) and shown in figure 6.4. This SOO was used as the reference slice because in our research group most experimental data has been acquired from this slice (van Donkelaar et al., 1999a; Gielen et al., 2000; Maenhout et al., 2001b). From the comparison in figure 6.4 between the model and the transverse cross-section of a TA muscle it is obvious that the geometry in the transverse direction is less realistic than in the other two dimensions. The transverse geometry is approximated by a 
rectangle, while the real geometry resembles a triangle.

a

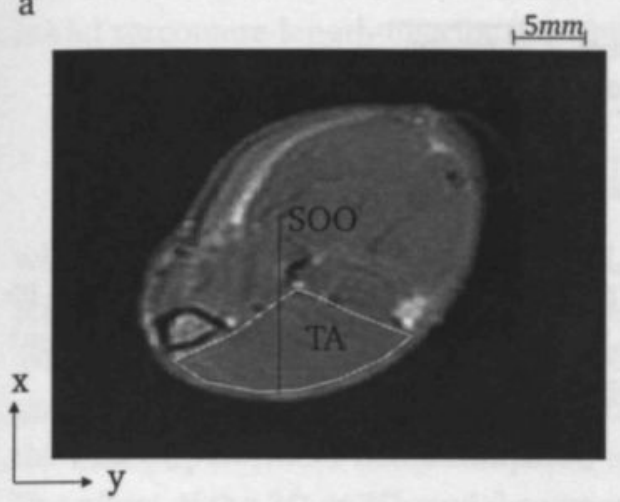

b

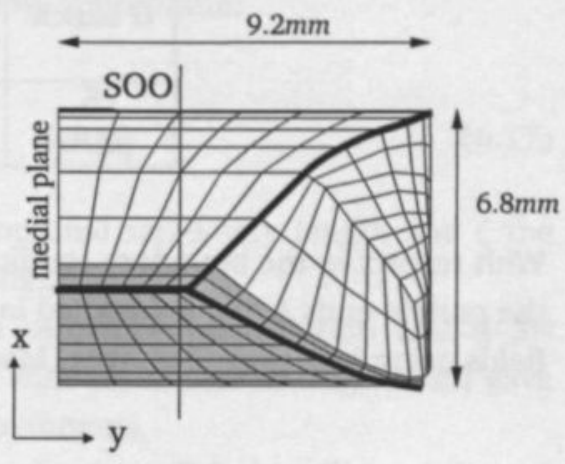

Figure 6.4: Representation of the TA in the sagittal-transverse (x-y) plane. a) Spin echo image of the central transverse slice with the definition of the slice of observation (SOO). The position of the TA is indicated by the white line. The $x$-direction coincides with the sagittal superficial-deep direction and the $y$-direction coincides with the transverse medial-lateral direction. b) $3 D$ model of the TA as already shown in figure 6.3, with the view-point in proximal-distal (-z) direction, and the definition of the SOO.

\subsubsection{Simulation settings}

The model parameters that have not yet been specified are; 1) the material constant $c_{a}$ determining the active stress, 2 ) the shear moduli determining the passive stiffness of the tissue, 3 ) the boundary conditions and 4) the sarcomere length distribution. The constant $c_{a}$ was set to $3.25 \mathrm{MPa}$ resulting in a maximum isometric stress of about $500 \mathrm{kPa}$, which is the upper boundary of this stress reported in the literature ranging from $100 \mathrm{kPa}$ to $500 \mathrm{kPa}$ (Fitts et al., 1991; van Leeuwen and Spoor, 1992; Trestik and Lieber, 1993; Abe et al., 1996).

Although some data about the passive muscle and tendon behavior is available in the literature (Bosboom et al., 2001b; Myers et al., 1998; Sasaki and Odajima, 1996; Yamamoto et al., 1999), shear moduli fitted to this data resulted in numerical convergence problems due to unrealistic large deformations. In order to simulate comparable strains to the measured ones (Maenhout et al., 2001b,a) the shear moduli were set as listed in table 6.2. 
Table 6.2: Shear moduli settings.

\begin{tabular}{|c|c|c|}
\hline$G$ muscle & $\begin{array}{l}G \text { proximal } \\
\text { aponeurosis }\end{array}$ & $G$ tendon \\
\hline $\begin{array}{c}5 \\
{[\mathrm{MPa}]}\end{array}$ & $\begin{array}{c}10 \\
{[\mathrm{MPa}]}\end{array}$ & $\begin{array}{c}20 \\
{[\mathrm{MPa}]}\end{array}$ \\
\hline
\end{tabular}

With respect to the boundary conditions it was decided to prescribe displacements to the muscle ends $A$ and $K$ (defined in figure 6.5) based on the measured displacement fields using MRI tagged images (Maenhout et al., 2001a) as:

$$
\begin{aligned}
A: \Delta y & =-0.2 \mathrm{~mm} \\
\Delta z & =0.4 \mathrm{~mm} \\
K: \Delta y & =-0.6 \mathrm{~mm}
\end{aligned}
$$

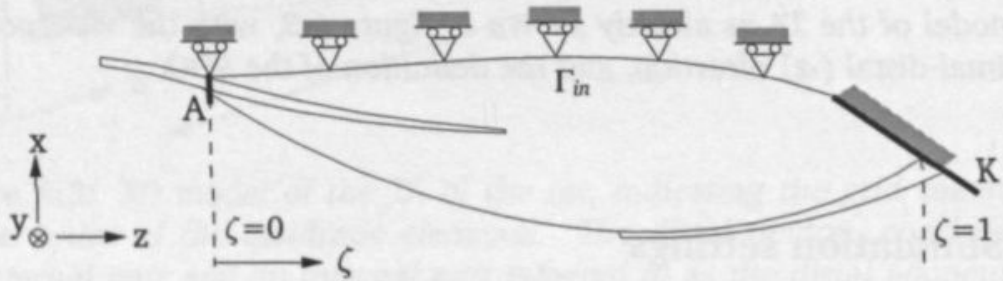

Figure 6.5: Boundary conditions: In all cases movement of the ankle (A) in $x$ direction and of the knee $(K)$ in $x$ and $z$ direction was suppressed. The movement of $\Gamma_{\text {in }}$ was suppressed in $\mathrm{x}$ direction. The $\mathrm{x}$-direction coincides with the sagittal superficial-deep direction, the $y$-direction with the transverse medial-lateral direction and the $\mathrm{z}$-direction with the longitudinal distal-proximal direction. All other boundaries were not suppressed.

It was assumed that friction along the sliding plane of the TA with the tibia $\left(\Gamma_{\text {in }}\right.$ boundary, figure 6.5) is low. To meet this assumption, the movement of $\Gamma_{\text {in }}$ was only suppressed in $\mathrm{x}$-direction. Movement of the distal tendon end (A, figure 6.5) was suppressed in $\mathrm{x}$ direction and the muscle and proximal aponeurosis at the knee $(\mathrm{K}$, figure 6.5) were suppressed in $\mathrm{x}$ and $\mathrm{z}$ direction.

The initial sarcomere length distribution is reported to be inhomogeneous, with the shortest sarcomeres at the muscles ends (Huxley and Peachey, 1961; Zuurbier and Huijing, 1993; Tidball, 1991). The exact sarcomere length distribution within the TA however is not known and a distribution with short sarcomeres at the muscles 
ends resulted in simulated displacement fells, whikh largely deviated from the experinemally observed fells (Msenhout et al, 2001a). The followizg nownalised initial sarcemere lenguth disuribution sppeared most approputatec:

$$
\frac{\text { Las }}{\text { Lape }}=0.05+1.755
$$

with $L_{\text {ig }}$ the initial sarcomere length, Lepe the eptimal sarcomere length and $\mathrm{Q}$ the scaled position from ankle to knee as defined in figure 6.5 .

The reference model with an inhomogeneous sareomere distribution and a 30 geometry (MSD) will be referred to as the model with parameter settings which gave the best displacement fields compared to the experiments.

The effect of the $2 \mathrm{D}$ or $3 \mathrm{D}$ model geometry on the simulation results will be evaluated just as the effect of the the sarcomere length distribution. The parameter settings of the three simulations to be evaluated are listed in table 6.3 .

Table 6.3: Summary of simulation settings. The initial sarcomere length distribution was homogeneous with $\frac{60}{6 \pi}=1.05$ or inhomogeneous, according to equation (6.17).

\begin{tabular}{|c|c|c|}
\hline $\begin{array}{c}\text { Type of } \\
\text { simulation }\end{array}$ & $\begin{array}{c}\text { Initial sarcomere } \\
\text { length distribution }\end{array}$ & Geometry \\
\hline I3D & inhomogeneous & 3D \\
H3D & homogeneous & 3D \\
I2D & inhomogeneous & 2D \\
\hline
\end{tabular}

\subsubsection{Experiments}

\section{MRI tagging}

In vivo 3D voxel by voxel displacement and strain maps were acquired non-invasively from two sets of orthogonal MR images with tissue tagging of the tibialis anterior muscle (TA) of rat during the plateau phase of an isometric contraction. Three male, 8 weeks old Wistar rats were used. The experimental setup, the exercise and imaging protocol and the method used to determine the displacements are described in detail in Maenhout et al. (2001a) (chapter 5).

\section{Video analysis of superficial markers}

Superficial displacements during isometric contraction were acquired by $3 \mathrm{D}$ videoanalysis of surface markers, which were attached to the free dissected muscle surface 
in muscle fiber direction from ankle to knee of the TA muscle of seven rats. Strains during the plateau-phase were calculated from the displacements. The experimental setup, the exercise protocol and the method used to determine the displacements and strains are described in detail in Maenhout et al. (2001b) (chapter 4). The local ethical committee approved both the video and MRI experiments.

\subsubsection{Comparing simulations and experiments}

In order to properly compare the simulated and measured strains using MRI tagged images and video analysis respectively, the simulated strains were determined from the displacement field in the same way as in the two experiments. Furthermore, the degree of discretization of the displacement field must be comparable.

The MRI measured 3D displacement field was determined on a 3D grid having an inter-grid distance of $1 \mathrm{~mm}$. Therefore, the simulated displacements were transformed by 3D linear interpolation to an identical 3D grid. Then, the deformation-gradient tensor $\boldsymbol{F}$ of each node was calculated from the displacements of the node and the surrounding $3 \times 3 \times 3-1=26$ nodes by a second order approximation method (Geers et al., 1996). Green-Lagrange strains were defined as:

$$
\mathrm{E}=\frac{1}{2}\left(\boldsymbol{F}^{T} \cdot \boldsymbol{F}-\boldsymbol{I}\right)
$$

and were computed from the deformation-gradient tensors $\boldsymbol{F}$.

The mean distance between the superficial markers used in the video analysis experiments was $2.3 \pm 0.6 \mathrm{~mm}$. The Green-Lagrange strain along the surface of the TA in approximately the mid-sagittal plane in fiber direction from ankle to knee was determined as:

$$
E_{s}=\frac{1}{2}\left(\left(\frac{l_{m}}{l_{m 0}}\right)^{2}-1\right)
$$

where $l_{m}$ is the distance between two markers during the plateau phase of the contraction and $l_{m 0}$ represents the distance at rest. In order to determine $E_{s}$ from the simulated displacements, first the displacements of the superficial middle nodes, which are assumed to be in the mid-sagittal plane, were interpolated to nodes with an inter-distance of $2.3 \mathrm{~mm}$. Next, $E_{\mathrm{s}}$ was determined according to equation (6.19). As an additional check of the model the muscle force in longitudinal direction at the distal tendon end A (figure 6.5) was computed from the mean stress of all the nodes 
on the surface of the tendon end A. This force was compared to the mean muscle force of the TA reported by Maenhout et al. (2000) (chapter 3) being $14.6 \pm 3.4 \mathrm{~N}$ $(n=7)$.

\subsection{Results}

\subsubsection{Simulations}

In figure 6.6 the 3D strain distribution of the reference simulations I3D (inhomogeneous sarcomere length distribution, 3D mesh) is shown. The sagittal and transverse strains are positive (about 0.03 ) in the mid-area of the TA, while the longitudinal strains are negative (about -0.05). At the muscle ends the opposite occurs; sagittal and transverse strains are negative, while the longitudinal strains are positive.
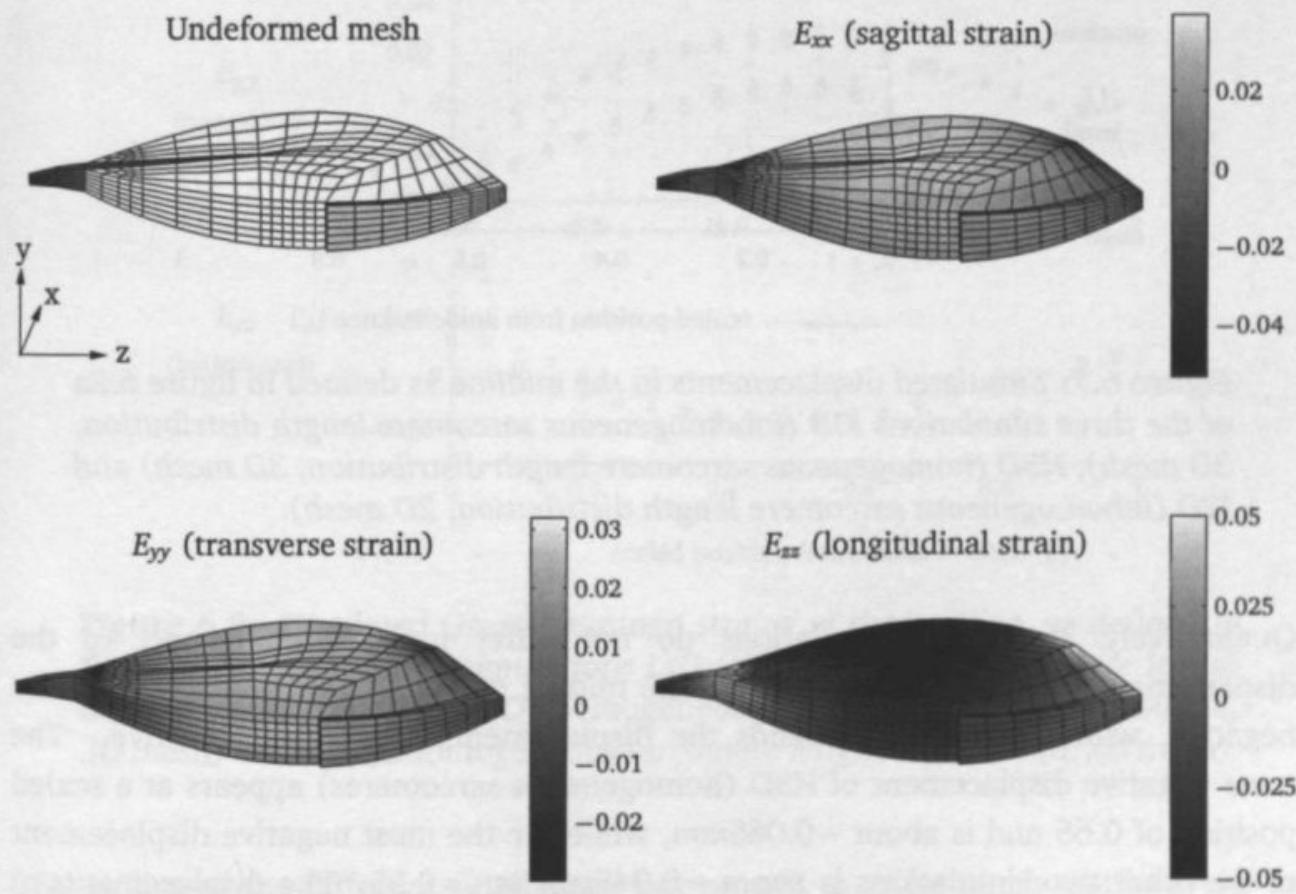

Figure 6.6: Simulated Green-Lagrange strains of the reference simulation I3D (inhomogeneous sarcomere length distribution and 3D mesh). 
The displacements and strains of the midline of the three different simulations are shown in figure 6.7 and 6.8 respectively.

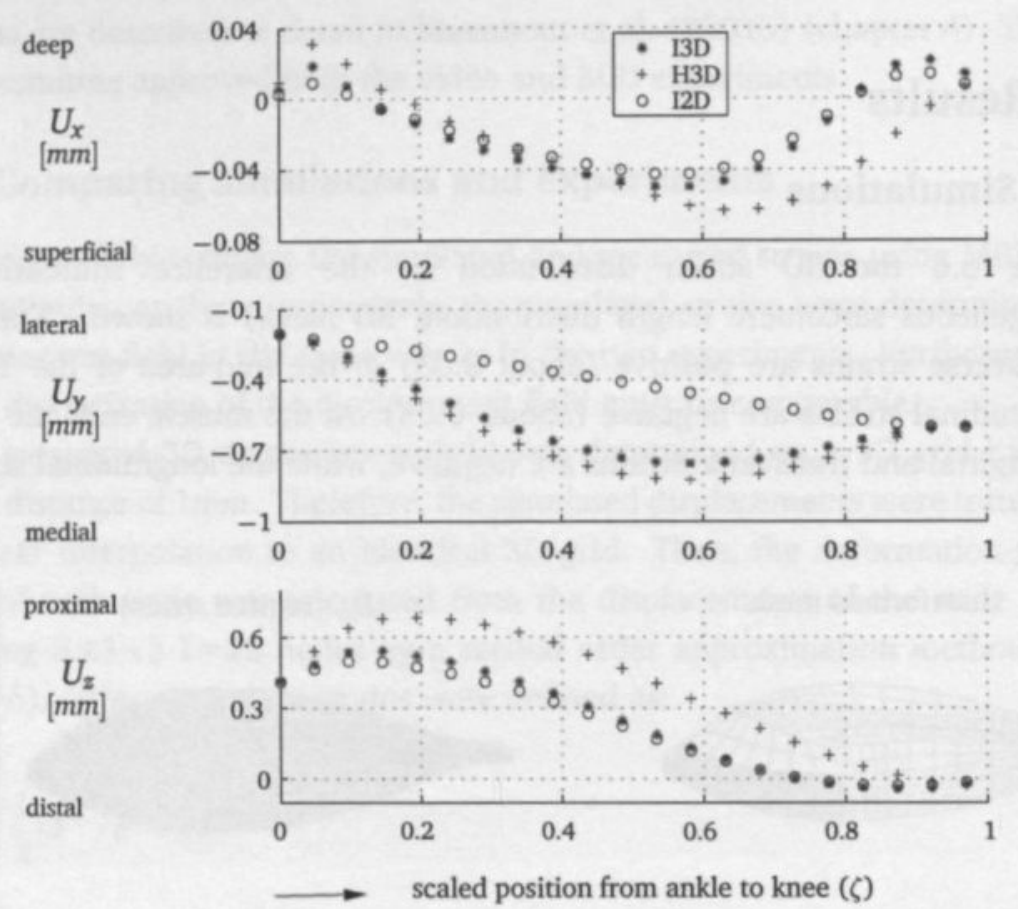

Figure 6.7: Simulated displacements in the midline as defined in figure 6.2a of the three simulations I3D (inhomogeneous sarcomere length distribution, 3D mesh), H3D (homogeneous sarcomere length distribution, 3D mesh) and I2D (inhomogeneous sarcomere length distribution, 2D mesh).

Qualitatively, the three simulations do not differ much with respect to the displacements in sagittal direction. In the middle of the TA the displacements are negative, while at the muscle ends the displacements are slightly positive. The most negative displacement of H3D (homogeneous sarcomeres) appears at a scaled position of 0.65 and is about $-0.065 \mathrm{~mm}$, while the the most negative displacement of the other two simulations is about $-0.045 \mathrm{~mm}$ at $\zeta=0.55$. The displacements in transverse direction are negative for all simulations, implying that the midline moves toward the tibia. The distribution and the values of the transverse displacements do not differ much for the different sarcomere length distributions (I3D and H3D); the most negative displacement of about $-0.8 \mathrm{~mm}$ appears at $\zeta=0.6$. The most prominent difference is the linearly decreasing displacement of I2D (2D) compared to the other two simulated displacement fields. Qualitatively, the displacements 
in longitudinal direction differ not much. Distally, the displacements first increase and from a scaled position of about 0.2 they all decrease in proximal direction. Distinctions are the more positive displacements over the whole range of scaled positions of H3D (homogeneous sarcomere length distribution). It is obvious that the differences between the 2D and 3D simulations (I3D and I2D) are only prominent for the transverse displacements which are maximally about $50 \%$ of the maximal displacement compared to $15 \%$ and $9 \%$ for the sagittal and longitudinal displacements respectively.

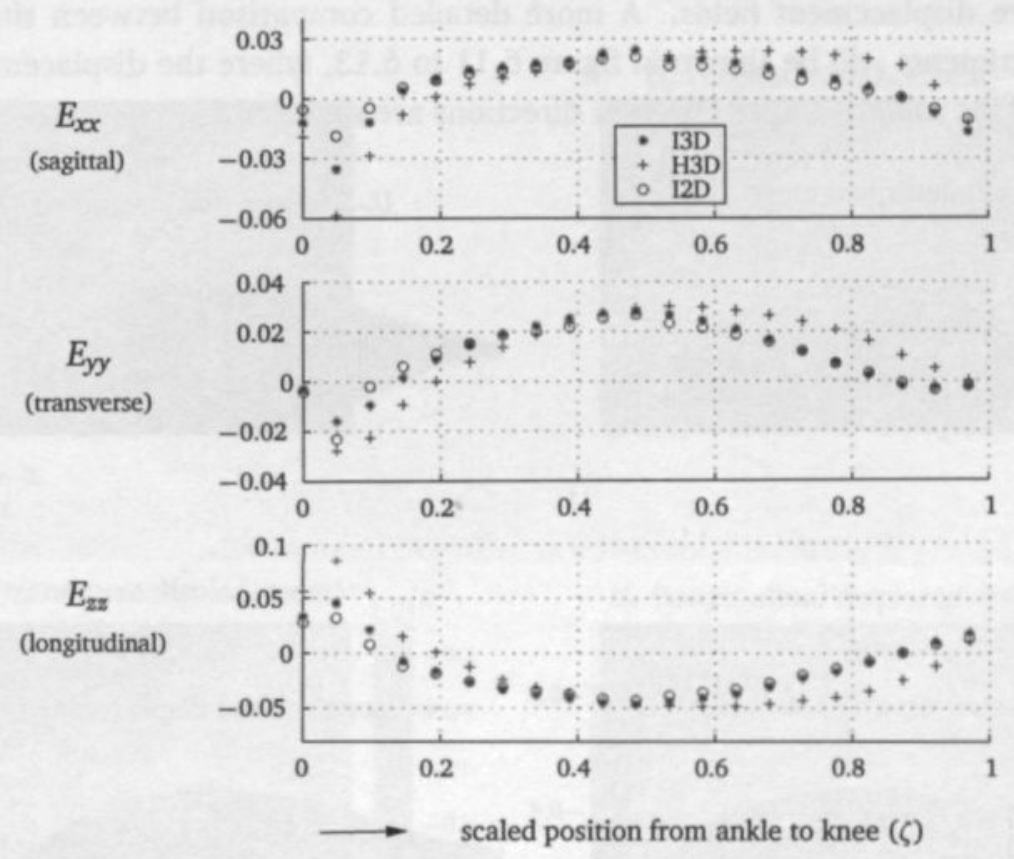

Figure 6.8: Simulated Green-Lagrange strains in the midline, as defined in figure 6.2a, of the three simulations I3D (inhomogeneous sarcomere length distribution, 3D mesh), H3D (homogeneous sarcomere length distribution, $3 D$ mesh) and I2D (inhomogeneous sarcomere length distribution, 2D mesh).

The strains of the different simulations appear not to differ much qualitatively. In the area from $\zeta=0.3$ to $\zeta=0.5$ also the quantitative values of the strains are similar (maximal difference 10\%). In the middle of the TA the sagittal and transverse strains are about 0.025 and the longitudinal strains about -0.05 . At the muscle ends the distal sagittal strains range from about -0.06 (H3D) to -0.005 (I2D) and the proximal sagittal strains range from -0.02 (I3D) to -0.01 (I2D). The distal transverse strains range from approximately -0.03 (H3D) to -0.005 (I3D) and the proximal 
transverse strains are about 0 . Finally the distal longitudinal strains vary between 0.03 (I2D) and 0.085 (H3D) and the proximal strains are about 0.015 .

\subsubsection{Simulation versus Experiment}

In this section the simulation results of the reference model I3D are compared to the experimental results. In figure 6.9 the simulated 3D displacement fields are shown, while in figure 6.10 the measured displacement fields in the SOO using MRI tagging are shown. The purpose of these figures is comparing the general qualitative displacement fields. A more detailed comparison between simulations and experiments will be shown in figure 6.11 to 6.13 , where the displacements and strains of the midline in the different directions are shown.
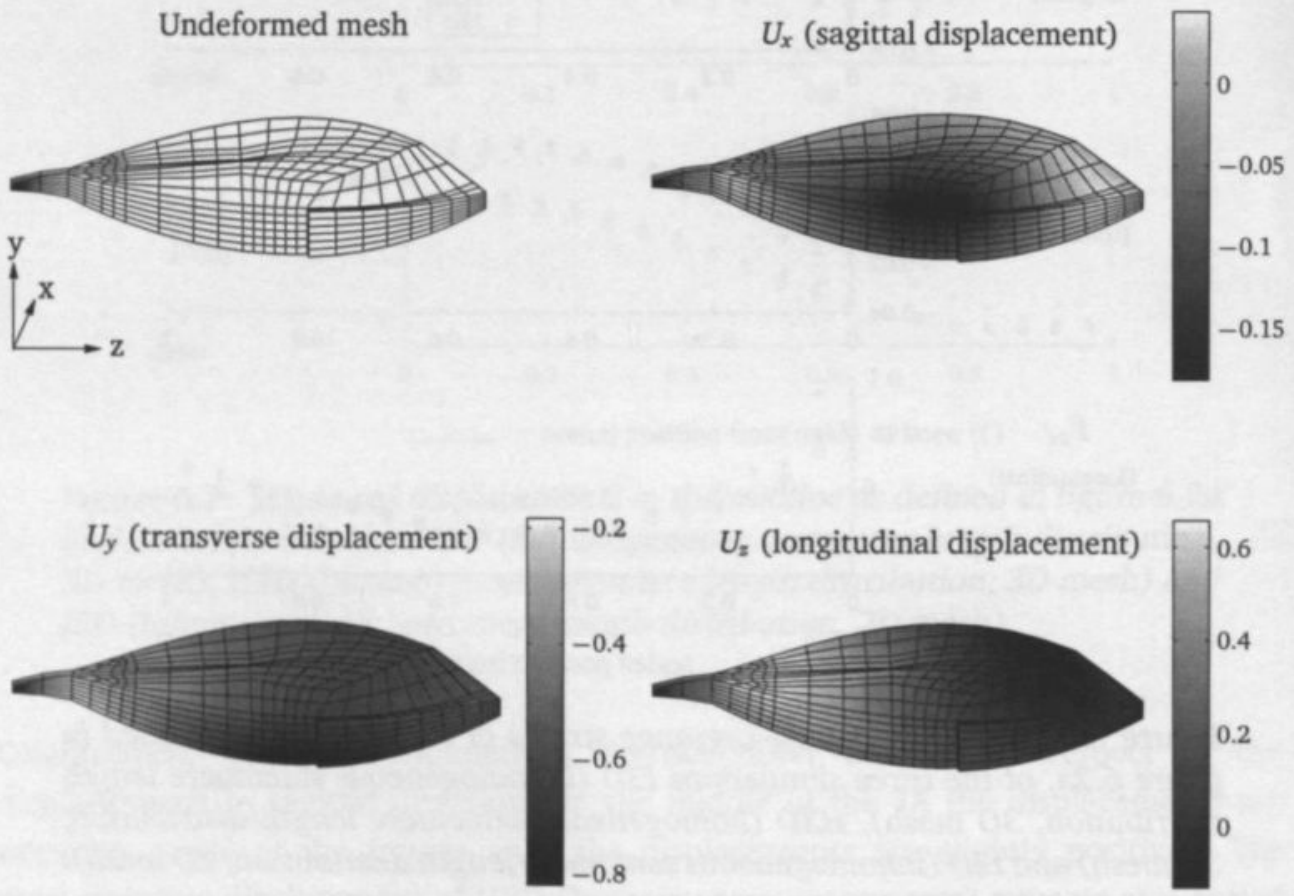

Figure 6.9: Simulated displacement field of the TA (reference simulation I3D, inhomogeneous sarcomere length distribution, 3D mesh). 
Comparing the simulated 3D displacement fields shown in figure 6.9 and the measured displacement fields in 6.10 shows that, for both the simulations and the measurements, the sagittal displacements are largest in the superficial middle area of the TA. The transverse displacements are negative for both simulations and measurements. For the simulation it holds that the proximal displacements are more negative than the distal ones. This is not observed in the MRI experiment, where the superficial middle area exhibits the largest negative displacements. The trend of the simulated and measured longitudinal displacement fields is again alike; proximal displacements are negative, while distal displacements are positive.
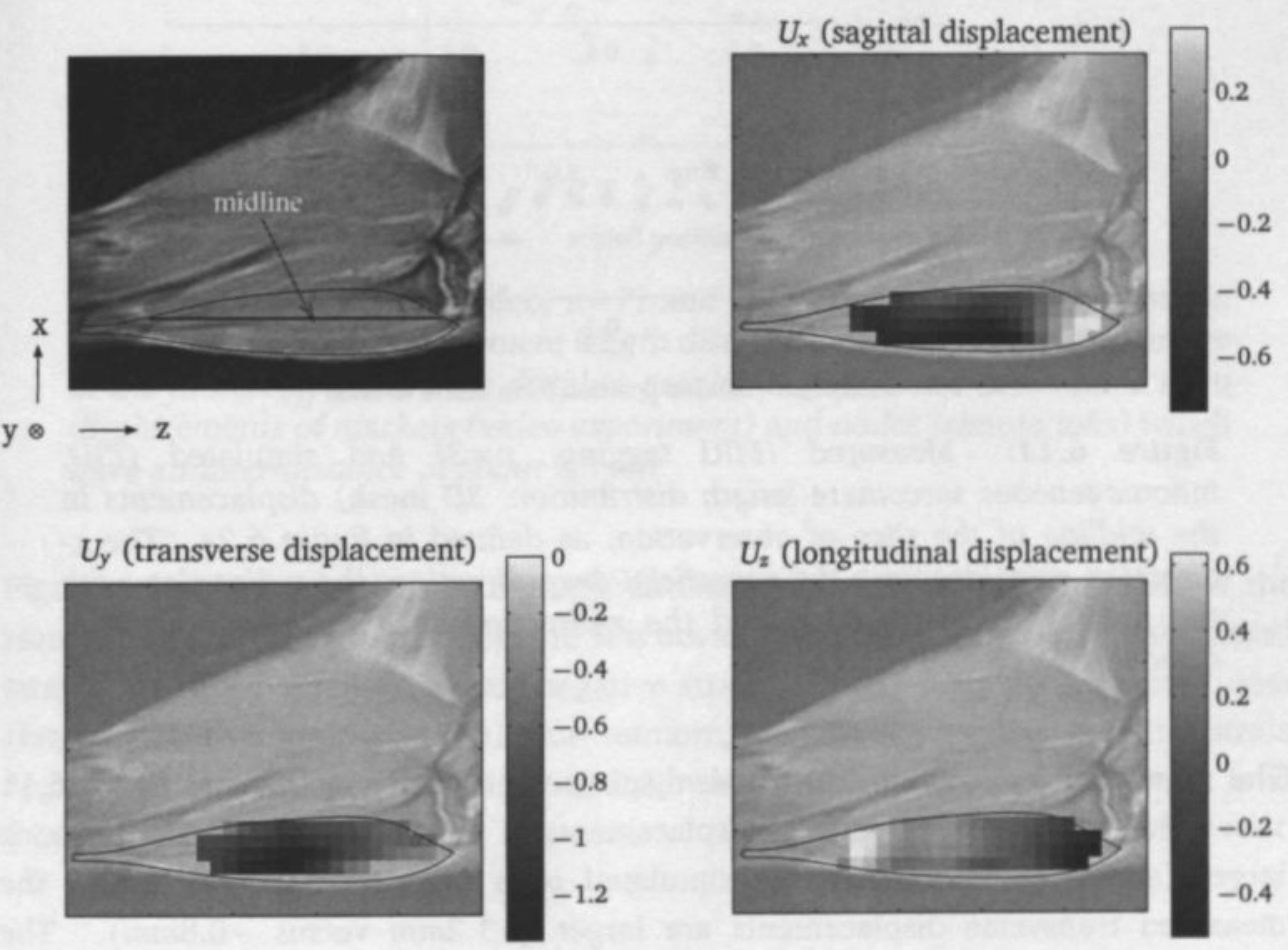

Figure 6.10: Measured displacement field of the $\mathrm{SOO}$, as defined in figure 6.4, of the TA of one rat using MRI tagged images. 

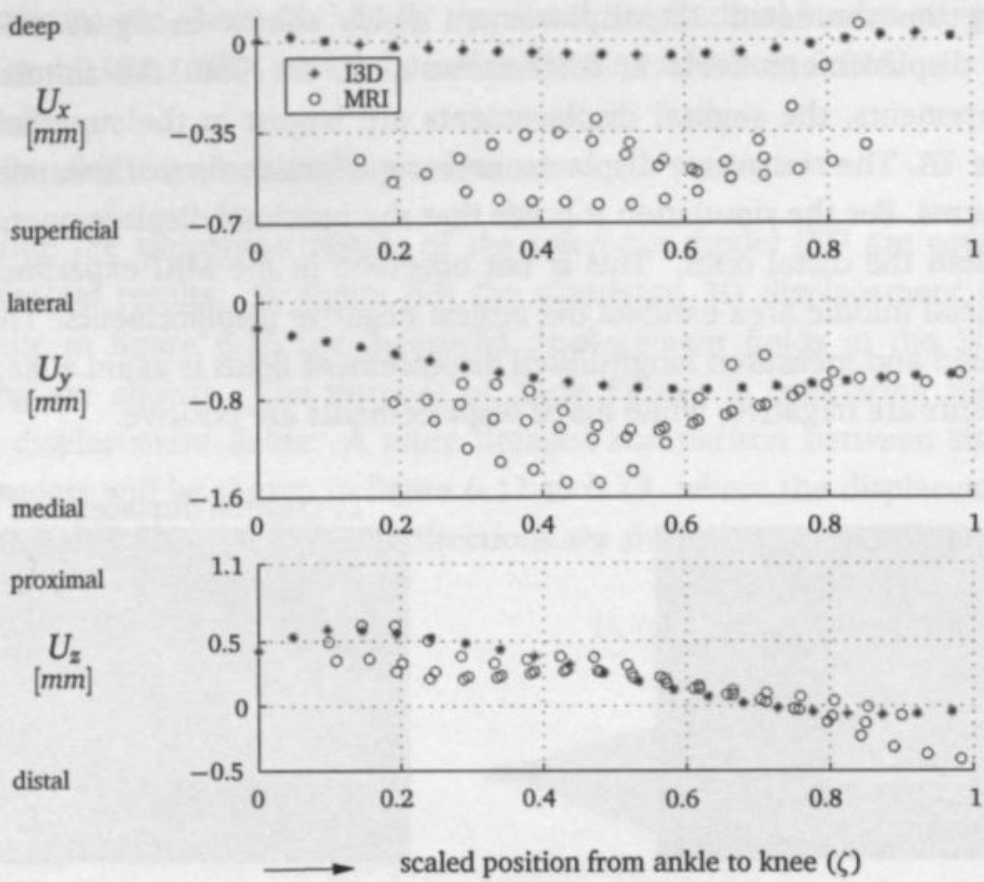

Figure 6.11: Measured (MRI tagging, $n=3$ ) and simulated (I3D, inhomogeneous sarcomere length distribution, 3D mesh) displacements in the midline of the slice of observation, as defined in figure 6.2a. The $\mathrm{x}$ direction coincides with the superficial-deep direction, the $y$-direction with the medial-lateral direction and the z-direction with the distal-proximal direction.

The more detailed comparison of the displacements in the midline in figure 6.11 shows that the measured sagittal displacements in the middle of the TA are much larger (about $-0.5 \mathrm{~mm}$ ) than the simulated ones (about $-0.08 \mathrm{~mm}$ ). Also the measured transverse displacements are larger $(-1.2 \mathrm{~mm}$ versus $-0.8 \mathrm{~mm})$. The longitudinal displacements are within the range of the measured displacements. 


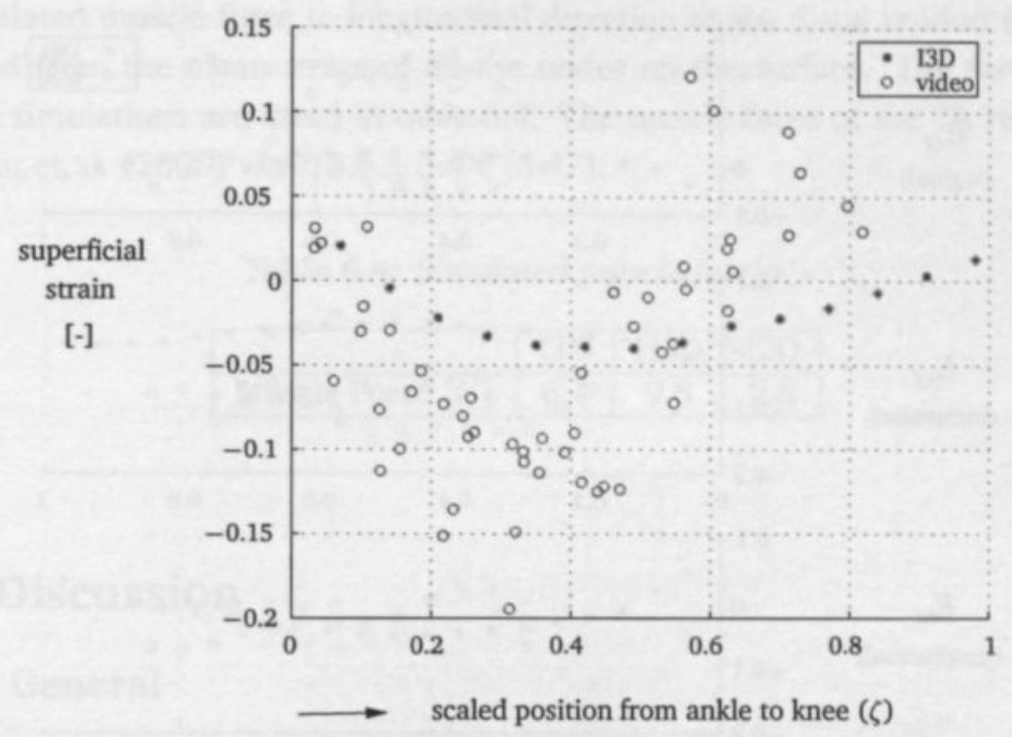

Figure 6.12: Measured (video, $n=7$ ) and simulated Green-Lagrange strain (I3D, inhomogeneous sarcomere length distribution, 3D mesh) at the surface of the muscle in a line from distal to proximal. Strains are determined from displacements of markers (video experiment) and nodes (simulations) which have an inter-distance of about $2.3 \mathrm{~mm}$.

Figure 6.12 shows the Green-Lagrange strains at the surface of the muscle of the simulations and the video experiment. It is obvious from this figure that the simulated strains are much smaller (largest negative strain $=-0.05$ ) than the measured ones (largest negative strain $=-0.15$ ). Furthermore, the largest simulated negative strain appears at a scaled position of about 0.5 , while the largest measured strain is at $\zeta=0.3$. 


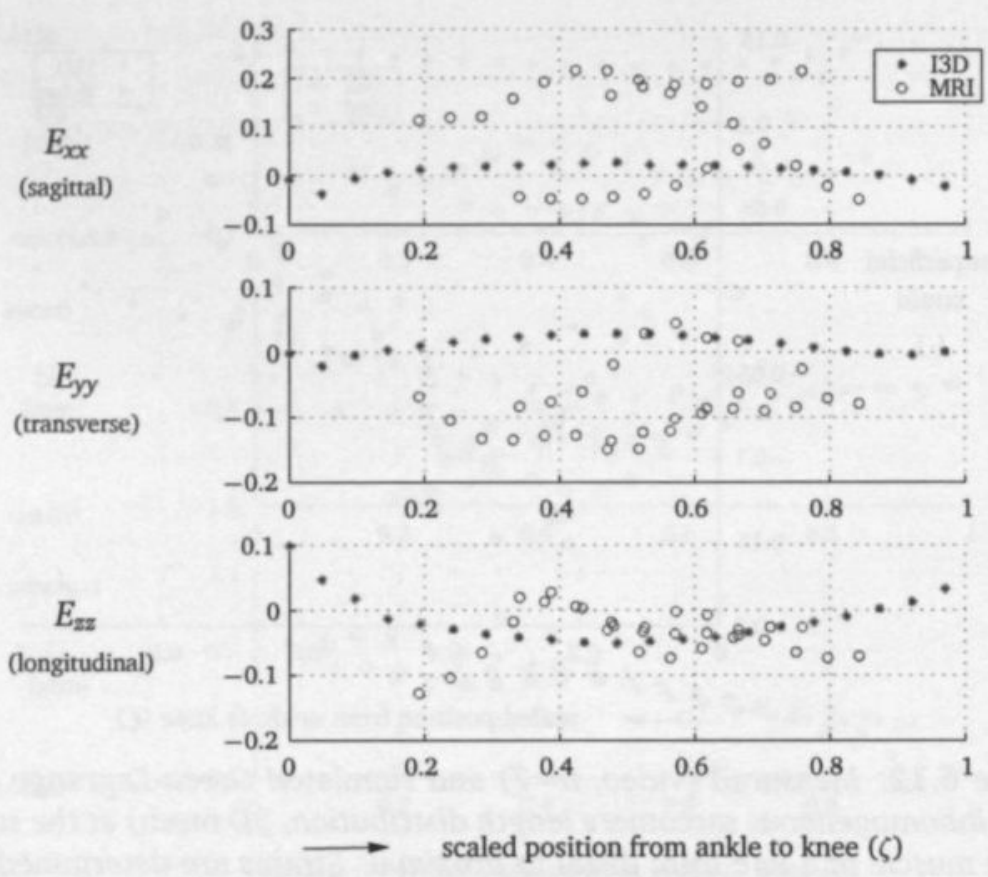

Figure 6.13: Measured (MRI tagging, $n=3$ ) and simulated Green-Lagrange strains (I3D, inhomogeneous sarcomere length distribution, 3D mesh) in the midline of the $S O O$ of the $T A$, as defined in figure 6.2a. $E_{x x}$ represents sagittal strain, $E_{y y}$ transverse strain and $E_{z z}$ represents longitudinal strain.

The measured and simulated Green-Lagrange strains in the midline of the SOO of the TA are shown in figure 6.13. It is most obvious that there is a large spread in the sagittal and transverse strains which were acquired from MRI tagged images. The largest difference is $150 \%$. The small simulated sagittal strains (about 0.02 ) are within the negative strains of one rat and the positive strains of the other two rats. The simulated transverse strain is positive (about 0.03 ) in the middle area of the TA, while the measured strains are negative (about -0.1 ). For both the sagittal and transverse strains no qualitative resemblance can be marked for the strain distribution. The values of the longitudinal strains are comparable; in the middle of the TA the strains are about -0.05 for both cases. However, the distribution differs. The simulated strains are negative in the middle and positive at the muscles ends, while at the position of 0.4 all measured longitudinal strains are about 0 . In proximal direction, to a scaled position of about 0.55 , they decrease to approximately -0.07 . 
The simulated muscle force in longitudinal direction at the distal tendon end $\mathrm{A}$ was computed from the mean stress of all the nodes on the surface. The forces of the different simulations are listed in table 6.4. The muscle force of the TA reported by Maenhout et al. (2000) was $14.6 \pm 3.4 N(n=7)$.

Table 6.4: Simulated muscle forces.

\begin{tabular}{|c|c|c|c|}
\hline & I3D & H3D & I2D \\
\hline Muscle Force $[N]$ & 6.4 & 9.8 & 2.8 \\
\hline
\end{tabular}

\subsection{Discussion}

\subsubsection{General}

In the present chapter, a 3D continuum model of a contracting muscle was presented. Its 3D geometry was loosely based on MRI data of rat TA muscle. Differences in displacement and strain between 2D and 3D geometries and between homogeneous and inhomogeneous sarcomere length distributions were evaluated. The differences between the 2D and 3D simulations were prominent only for the transverse displacements. Proximally, the homogeneous simulation (H3D) showed a more negative longitudinal strain, while distally the strains were more positive. The opposite effect was observed for the strains in the cross-fiber directions. Simulations were compared with experimental force, displacement and strain data, the latter obtained by MRI tagging and video analysis of surface markers. The tendon force equaled $6.4 \mathrm{~N}$, which was lower than the measured force of $14.6 \pm 3.4 \mathrm{~N}$ $(n=7)$ (Maenhout et al., 2000). The simulations could qualitatively describe the experimentally acquired displacement fields from the MRI tagged images and to some extend the longitudinal strain distributions. However, quantitative agreement was not present for either displacement or strain fields. Obvious differences occurred in the transverse strains in the distal area at a scaled position of 0.4: simulated strains were positive and measured strains were negative. The opposite effect was observed for longitudinal strains.

\subsubsection{Model parameters}

\section{Geometry}

Although the geometry was based on MRI-data, some important deviations from the anatomical morphology were present. The cross section was modeled square-shaped, 
while it is triangular. Furthermore, the transversal size of the distal aponeurosis was larger than in reality. Also the fiber direction especially near the distal aponeurosis showed deviations up to $15^{\circ}$ Bogaerds (1997). Since it is known from studies in the field of cardiac deformation (Bovendeerd et al., 1994; Rijcken et al., 1997, 1999) that small changes in the fiber direction can cause large changes in the strain fields, the fiber direction is supposed to be an important parameter.

A more realistic geometry can be constructed from existing high resolution MRI and Diffusion Tensor Imaging data (van Donkelaar et al., 1999a). Obviously, such a geometry is supposed to improve the agreement between measurements and simulations.

\section{Boundary conditions}

Displacements to the muscle ends were prescribed based on the measured displacement fields. However, the applied displacements at the muscle ends were extrapolated from MRI displacements in the mid-field of the muscle. For future investigations, the displacements of knee and distal tendon should be acquired.

Transverse displacements in the medial plane were not suppressed (see figure 6.4) to allow superficial, transverse displacements, as were measured near the tibia. Since in the the animal model the deeper tissue is suppressed by the tibia, deeper displacements near the tibia are overestimated by the model. Implementation of contact boundary conditions representing the sliding of the TA along the tibia in combination with a more realistic triangular cross section, might diminish this discrepancy between simulated and measured displacements.

\section{D Passive stiffness}

In these simulations, muscle tissue was described with a linear, isotropic material model. In order to simulate similar quantitative longitudinal displacements and strains compared to the experiments, the shear modulus $\mathrm{G}$ describing the behavior was chosen to be $5 \mathrm{MPa}$. This is comparable with the tangent modulus of Bosboom et al. (2001b) at strain values of $15-20 \%$, where a zero strain value was defined as the in situ situation, but much higher than the initial stiffness. It should be noted that Bosboom et al. (2001b) measured transverse properties assuming isotropy. Myers et al. (1998) reported an initial modulus in fiber direction ranging from $0.1 \mathrm{MPa}$ $0.7 \mathrm{MPa}$. The relatively high passive stiffness needed to give the best agreement with the experimental data may be explained by non-linear transversely isotropic material behavior. An other possible explanation is an extra passive stiffness during contraction due to structural changes of the cytoskeleton.

The shear modulus of the distal tendon and proximal aponeurosis of the TA was 
chosen to be $20 \mathrm{MPa}$ and $10 \mathrm{MPa}$ respectively. These values are about ten times smaller than values reported in the literature ranging from 100 to $200 \mathrm{MPa}$ (Sasaki and Odajima, 1996; Quapp and Weiss, 1999; Yamamoto et al., 1999). To avoid a high aspect ratio of the three dimensional elements, the thickness of the proximal aponeurosis was modeled thicker than the real proximal aponeurosis of the TA. The volume of the distal tendon is also larger than in reality, due to the transverse dimensions of the model. The low moduli therefore assure a more realistic overall stiffness. Furthermore, the values enabled the simulation of strains comparable to the measured strains.

\section{Sarcomere length distribution}

The two sarcomere length distributions that were employed showed that the homogeneous distribution resulted in a shift in proximal direction of the sagittal displacements and larger positive longitudinal displacements. Proximally, the inhomogeneous simulation (I3D) showed less shortening (less negative longitudinal strain) in approximately fiber direction, while distally the fibers lengthened less (less positive longitudinal strain). The opposite effect was observed for the strains in the cross-fiber directions. These observations could be expected since the proximal longer sarcomeres at the descending limb of the length-tension curve of I3D produce lower active stress. Therefore the proximal sarcomeres will shorten less than the distal ones. Experimentally, proximal displacements in longitudinal direction were negative (moving in distal direction) which can be explained by stronger sarcomeres in distal areas. Simulations with short distal sarcomeres $(\mathrm{ls} / \mathrm{lsopt}=1)$ and linearly increasing sarcomeres in proximal direction (maximum $\mathrm{ls} / \mathrm{lsopt}=1.73$ ) showed indeed negative proximal displacements in longitudinal direction. In order to simulate comparable displacements in longitudinal direction to the measured displacements, the initial sarcomere length distribution was employed according to equation (6.17). During pilot experiments Tobie (1997) measured the sarcomere length distribution of the TA using a laser diffraction technique. The results agree with the distribution used in the model. However, the results of Tobie (1997) were questioned because the variation of sarcomere lengths was much larger (35\%) than the in vivo acquired values found in the literature (5\%) (Kawai and Kuntz, 1973; Lieber and Baskin, 1983). This implies that there are probably other parameters, which are yet unknown, that could explain the negative longitudinal displacement in the proximal area of the TA.

\subsubsection{Simulations versus experiments}

The comparison of the computed 3D displacement fields and the measured displacement fields (see figure 6.9 and 6.10) showed that the displacements were 
qualitatively similar. However, the values of the measured sagittal and transverse displacements in the middle of the TA are much larger than the simulations (see figure 6.11). The longitudinal displacements were within the range of the measured displacements. The differences can be caused by the lack of detail in the 3D geometry. Especially the 3D geometry needs improvement. Moreover, as mentioned previously, the boundary conditions are uncertain, as well as the constitutive behavior.

Compared to the measured strains using video analysis, the simulated and MRI strains were smaller (largest negative strain -0.05 versus -0.15 ). However the distribution of the video and simulated strains is comparable; shortening in the middle of the muscle and lengthening at the ends. The difference may be explained by the preparation of the muscle surface to attach the markers prior to the contractions. The skin and stiff fascia was removed, which probably caused swelling of the resting muscle resulting in larger deformation during contraction.

The strains determined from the MRI tagged images were quite different from the simulated strains. In the distal area $(\zeta= \pm 0.4)$ the measured transverse strains were negative and the longitudinal strains were positive, while the simulations showed the opposite effect. This may be caused by the fiber directions in the 3D model, which were not accurately incorporated in especially the transverse direction.

Finally, it should be noted that the inter rat variation of especially the sagittal and transverse strains hampered the drawing of conclusions regarding the comparison between model and experiments.

\subsubsection{Concluding remark}

The study presented in this chapter should be interpreted as a first step towards the development and validation of realistic 3D muscle models. A set of model parameters was presented enabling good qualitative prediction of the MRI displacement fields. Quantitatively, large differences were found. Further improvement of the model is needed for more accurate description of the measured displacement and strain fields. Based on the findings described in this chapter, it can be concluded that the focus in future studies should be on the following parameters: the 3D geometry and fiber directions, the boundary conditions, the passive constitutive behavior, and the local sarcomere length distributions. These parameter studies can lead to better understanding of the existing differences between measurements and simulations and of the influence of various model parameters on the deformation of contracting muscle. A suitable procedure to identify uncertain model parameters would be a numerical/experimental approach (Oomens et al., 1993). Although further improvement of the presented model is needed, it should be emphasized that the $3 \mathrm{D}$ model as it is offers a solid tool to investigate the muscle mechanics. 


\section{Chapter 7}

\section{Discussion}

\subsection{Recapitulation}

To study heterogeneous aspects of skeletal muscle contraction on different levels (e.g. tissue level, cell level), continuum models like the model of Gielen et al. (2000) of the rats TA muscle are a solid tool. The purpose of the present thesis was 1) to acquire experimental data enabling the validation of continuum models of the rats TA muscle, and 2) to improve the 2D model of Gielen et al. (2000) especially with respect to its geometry. Using the sagittal plane model, various numerical output parameters can be evaluated (e.g. muscle force, displacements, fiber strain, strain gradients). Therefore, an approach was chosen incorporating different measuring techniques to collect an extensive validation data set.

The first experiments, described in chapter 3, were focused on parameter identification of the so-called distribution-moments (DM) approximated two-state Huxley cross-bridge model. This model was used to describe the contractile properties in the continuum model. The identification involved fitting of a 1D model of the muscle-tendon complex to experimental isometric muscle torque data at different stimulation frequencies. The results indicated that the identified parameters of the DM model enable a good description of the muscle torques of the TA of the rat at different stimulation frequencies. The 1D model however overestimated the experimentally observed rate of torque relaxation, as was discussed extensively in chapter 3.

3D superficial marker displacements during electrical stimulation of an isometrically contracting TA muscle of a rat were acquired by 3D video analysis (chapter 4 ). It was evaluated whether or not the strain distributions depend on 1) the muscle force 2) the joint angle of the rats ankle. Furthermore, it was evaluated how the strain distributions varied during onset and relaxation of the muscle force. The strains 
during the plateau-phase calculated from the marker displacements were strongly dependent on longitudinal position. The results also indicated that less than $20 \%$ muscle force variation does not have a significant influence on local deformation. Furthermore, variation of the ankle angle of about $30^{\circ}$ caused a strain difference of 0.01 (independent of the longitudinal position) compared to a strain range in longitudinal direction of 0.17 . Therefore, it was concluded that small differences in the angle of the rats ankle (order $5^{\circ}$ ) are not supposed to significantly influence the local deformation. During onset and relaxation of the muscle force unexpected phenomena were observed. At the start of the stimulation, while the force increased, proximal regions shortened faster than distal regions. During force relaxation the distal regions showed additional shortening, while the proximal regions lengthened before the initial state was reached. From these phenomena, it was hypothesized that the propagation of the action potential along muscle fibers originating from the motor end-plates strongly influences the time course of the local strain distribution.

MRI-tagging was employed, as described in chapter 5, to acquire the internal 3D tissue deformation during the plateau phase of a fused tetanus. 3D Displacement and strain maps of about $70 \%$ of the muscle volume were acquired from two sets of orthogonal MR images with tissue tagging of an isometrically contracting TA muscle of a rat. To evaluate the suitability of superficial strains as a measure of deep strains, sagittal strain gradients were determined from the displacement maps. Also transverse strain gradients were determined to evaluate the suitability of sagittal plane models to describe the distribution of mechanical quantities within the muscle. The largest differences over a distance of $1 \mathrm{~mm}$ in sagittal and transverse direction of the strain in a particular direction were about $50 \%$ of the maximum absolute strain in that direction, implying strong heterogeneous strain distributions within the muscle. It was concluded therefore, that 1 ) superficial strains are not a good measure for deeper strains and 2) to study 3D muscle mechanics during contraction, models incorporating realistic 3D geometries are needed.

Chapter 6 describes the extension of the 2D sagittal plane geometry of Gielen et al. (2000) of the rat tibialis anterior muscle to a more realistic 3D geometry. Furthermore, model simulations of strain and displacement fields are compared with the acquired experimental data set presented in this thesis. The finite element method was used to solve the conservation laws of the extended 3D continuum model. In contrast to Gielen et al. (2000) an Updated Lagrange formulation (van Oijen et al., 2001) instead of a Total Lagrange approach was used to solve the equations. A new solid element developed by van Oijen et al. (2001) was employed to solve the field equations. This element improved the robustness of the computations and the ability to deal with incompressible behavior. It appeared that the differences between the 2D and 3D simulations were only prominent for the transverse displacements. 
Furthermore, the initial sarcomere length distribution was varied (see table 6.3, chapter 6), which especially influenced the displacements and strains in longitudinal direction.

From the step towards model validation using the acquired experimental data, it became clear that the simulations could qualitatively describe the experimentally acquired displacement fields from the MRI tagged images and to some extend the longitudinal strain distributions. The inter rat variation of the strains determined from MRI tagged images complicated the drawing of conclusions concerning the comparison of strains between model and experiments.

\subsection{General discussion and recommendations}

\subsubsection{Video analysis}

The video experiments showed that torque deficits up to $20 \%$ of the initial value do not change the deformations, assuming that the 'fatigue' is evenly distributed throughout the muscle. Fatigue in this context means decreased capability to generate active force at maximum stimulation. Strain differences due to a variation of the angle of the rats ankle joint (optimal angle versus maximal plantar flexion) were small (about 0.01 compared to a maximum difference in strain of 0.17 along the muscle fiber) and the distribution of the strains was not influenced by the ankle angle. These findings may be important for developing damage inducing exercise protocols, which may be used to find the relation between local damage and local mechanical parameters. Muscle fatigue causing a $20 \%$ decrease of muscle force can be allowed and small differences in the initial angle of the rats ankle during a damaging protocol do not have a large influence on the strain distributions. Besides the relevance for developing exercise protocols, the experimentally determined relation between local strains and muscle force and muscle length respectively, can serve as validation data for continuum models.

From the measured strain distributions during onset and relaxation of the muscle contraction it was hypothesized that the propagation of the action potential along muscle fibers originating from the motor end-plates strongly influences the time course of the local strain distribution. Because the proximal regions shortened faster at the start of the stimulation and showed lengthening during torque relaxation, the motor end-plates were supposed to be situated proximally. To test this hypothesis, a double immunofluorescence assay was performed on sections of a rat TA with $\alpha$ bungarotoxine and an antibody against fibronectin. Nuclei were visualized using DAPI. From immunofluorescence microscopic pictures, as shown in figure 7.1, it became clear that the motor end-plates are indeed situated near the knee at a scaled 
position of about 0.7 . The hypothesis can be further investigated by incorporating inhomogeneous activation of the muscle fibers in a continuum model.

\subsubsection{MRI tagging}

It was shown in this thesis that the developed MRI tagging technique combined with the analysis method involving one dimensional signal correlation offers a powerful tool to measure in vivo 3D muscle deformation. The employed method was based on techniques employed in the field of cardiac deformation studies (Kuijer et al., 2000; Thomas and McVeigh, 1997; Alistair and Young, 1996). In these studies discrete displacement maps were obtained at the location of the tags. An easy and reliable method was used here, increasing the resolution to 3D voxel by voxel displacement mapping.

From the MRI experiments it was concluded that superficial strains are not a good measure for deeper strains because the variation per $\mathrm{mm}$ of the strain in sagittal direction was approximately $50 \%$ of the maximal strain. Furthermore, deformation in the transverse direction could not be neglected compared to the other two directions. This implies that to study 3D muscle mechanics during contraction, models incorporating realistic 3D geometries are needed as well as measuring techniques enabling the acquisition of 3D maps of mechanical quantities within the muscle. Both such a model and measuring technique were presented in this thesis.

\subsubsection{Model validation}

The contraction model used to describe the active stress within the muscle tissue was based on a two-state Huxley cross-bridge theory, including the calcium activation dynamics according to Zahalak and Ma (1990). This inclusion was an improvement compared to the model of Gielen (1998) and enables future investigation of metabolic processes. The identified parameters of the contraction model enable a good description of the measured muscle torques of the TA of the rat at different stimulation frequencies (chapter 3). The two-state Huxley contraction model is therefor believed to be sufficient for the description of contractile behavior in continuum models of whole muscle during isometric contractions. The $1 \mathrm{D}$ model however overestimated the experimentally observed rate of torque relaxation, which may be caused by spatial effects. These effects can be investigated in the future with continuum models.

A unique set of experimental data was acquired for model validation purposes. A comparison of the 3D continuum model with the experimental data showed that the experimentally acquired displacement fields from the MRI tagged images and to some extend the longitudinal strain distributions could qualitatively be described. 
However, the model needs further improvement to accurately describe the measured displacement and strain fields. The focus in future studies should be on the following parameters: the 3D geometry and fiber directions, the boundary conditions, the passive constitutive behavior, and the local sarcomere length distributions. For an extensive discussion of these parameters the reader is referred to section 6.4 .2 of chapter 6. Although further improvement of the model is needed it should be emphasized that the 3D model as it is offers a solid tool to investigate and improve the understanding of muscle mechanics during contraction.

Since it is known from studies in the field of cardiac deformation (Bovendeerd et al., 1994; Rijcken et al., 1997, 1999) that small changes in the fiber direction can cause large changes in the strain fields, the fiber direction is supposed to be an important parameter. From immunofluorescence microscopic pictures of the mid-sagittal plane of the TA muscle (figure 7.1), it is obvious that not all fiber directions are in the plane. This is in contradiction with the assumed fiber field in the 3D model. A more realistic fiber field can be constructed from existing Diffusion Tensor Imaging data (van Donkelaar et al., 1999a). Furthermore, the constructed 3D fiber field can be cross-checked by using results from immunofluorescence microscopic pictures as shown in figure 7.1.

The passive stiffness of the muscle tissue was described with a linear, isotropic material model. The required value for the shear modulus to simulate strains comparable to the experimental strains was about ten times larger than expected from values for the initial modulus found in literature (Bosboom et al., 2001b; Myers et al., 1998). The modulus was comparable with the tangent cross-fiber modulus of Bosboom et al. (2001b) at strain values of $15-20 \%$, where a zero strain value was defined as the in situ situation. This relatively high passive stiffness may be explained by non-linear transversely isotropic material behavior. An other possible cause can be a different passive stiffness during contraction, due to micro structural changes within the sarcomeres. To test this hypothesis the experiments of Bosboom et al. (2001b), who performed transverse compression tests on rat TA muscles at rest, should be repeated on a tetanized muscle. The $3 \mathrm{D}$ continuum model can be fitted to the data of the compression experiments at rest. If the measured resistance against compression during contraction appears higher than simulated with the fitted model, evidence will be given for an additional passive stiffness during contraction.

Especially the variation of the measured sagittal and transverse MRI strains between the three rats (see figure 5.9, chpater 5) complicated the drawing of conclusions regarding the comparison between model and experiments. For the time being the cause of this variation remains unclear and future research will be needed for better understanding. 


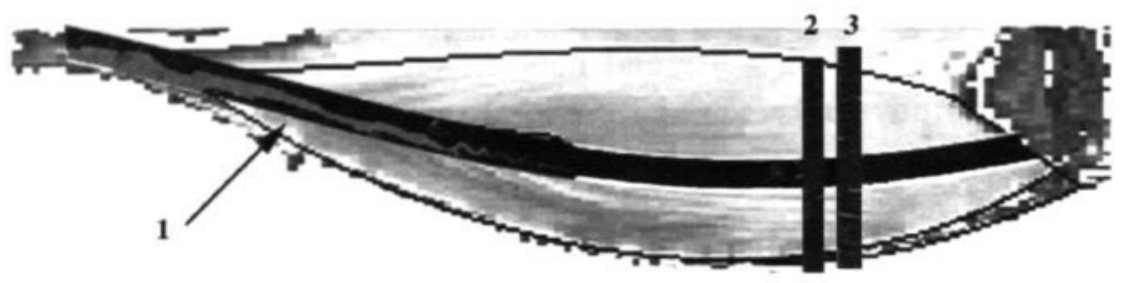

1

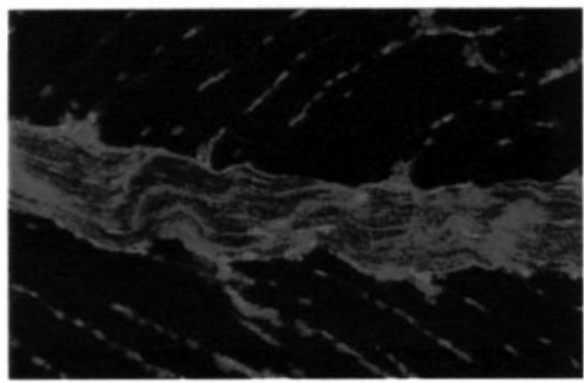

2

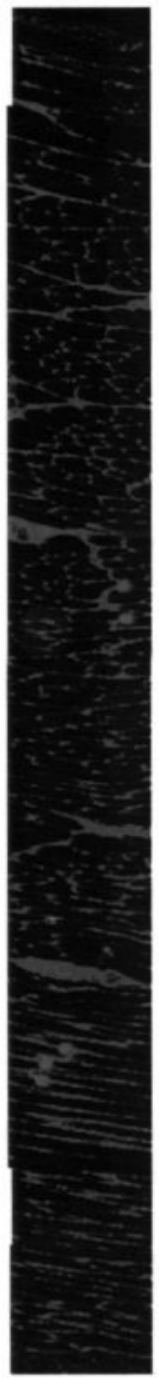

3

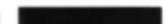

4
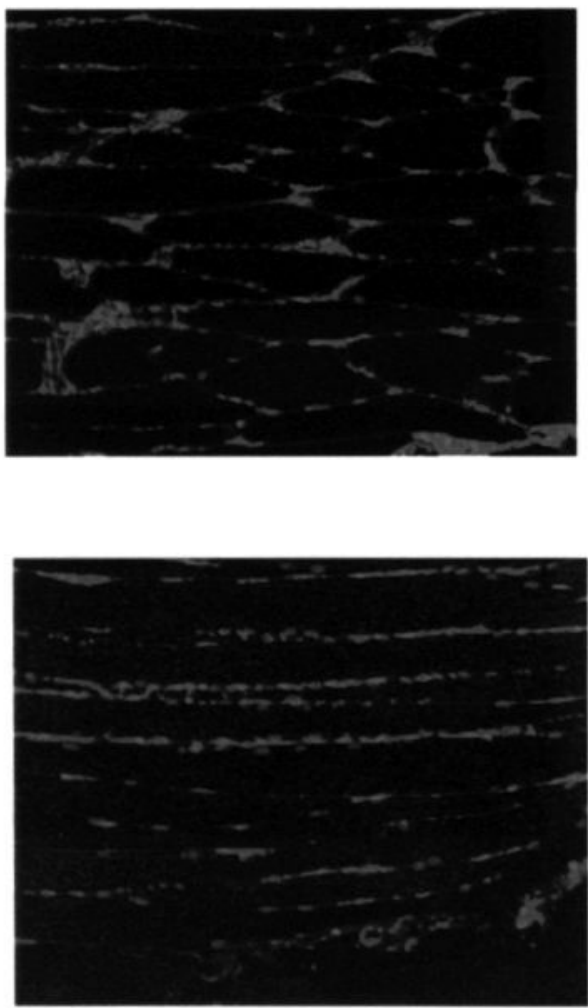

4

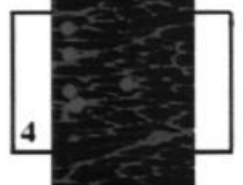


Figure 7.1: MR image of the mid-sagittal cross section of a TA muscle of a rat combined with immunofluorescence (IF) microscopic pictures of the muscle. A double immunofluorescence assay was performed on $5 \mathrm{~mm}$ frozen and dried sections with $\alpha$-bungarotoxine (Alexa 594, 1:400) and an antibody against fibronectin (polyclonal rabbit antiserum directed against fibronectin, Sigma; 1:250 plus goat anti-rabbit-Ig-FITC (SBA; 1:80)) embedded in Mowiol. Nuclei were visualized using DAPI. Three IF picture were composed by combination of a large number of images at $40 \times$ objective, one longitudinal and two transverse. Green is fibronectin: aponeurosis and border of muscle cells; blue is DAPI: nuclei; and red $\alpha$-bungarotoxine: motor end-plates. 1) Detail of the internal distal aponeurosis showing pinnate insertion of muscle fibers. 2) and 3) Enlargements of the two transverse IF pictures showing the position of motor end-plates (large red dots). 4) Detail of middle part of 3 showing five motor end-plates. It is also visible that the deeper fibers are at a large angle with the plane. 5) Detail of superficial part of 3 , the superficial proximal aponeurosis is faintly visible. It is clearly visible that, superficially, the direction of the muscle fibers is mainly in the plane (individual fibers are visible over the entire width of the view).

An other complicating factor was the difference found in the superficial fiber strains measured with the video analysis (figure 6.12 , chapter 6 ) and the most superficial fiber strains acquired with the MRI tagging technique (strain distributions were comparable with those shown in figure 6.13 , chapter 6 ). The largest negative video strains were -0.15 , while the MRI strains were maximally -0.07 . Furthermore, the largest negative video strain was found at a scaled position of about 0.3 , while the MRI strains were 0 at a scaled position of 0.4 and decreased in proximal direction towards a scaled position of about 0.55 . Although the video strains were measured most accurate (error less than 0.005 , versus MRI error less than 0.01 in the mid-field), they may severely be influenced by the preparation of the muscle surface to attach the markers prior to the contractions. The skin and stiff fascia was removed, causing some bulging of the resting muscle, which may result in an altered deformation during contraction. The shift of the maximum shortening in distal direction during the video experiments remains unexplained. By acquiring deformation with MRI-tagging of a TA muscle of which the skin and fascia are removed, the effect of the removal can be investigated by comparing the results with the deformation of the in situ TA muscle. In future utilization of the experimental data set for validation purposes, it should be taken into account that the video and MRI data set were acquired under different experimental conditions. Future parameter studies of the 3D continuum model (e.g. modeling the muscle with intact fascia, applying transverse passive compression prior to contraction) may lead to better understanding of the differences between video and MRI data. 


\subsection{Towards damage and adaptation}

Eventually the presented research must lead to better understanding of damage and adaptation processes as a result of mechanical loading. The next step in this direction should be the testing of the hypothesis that local mechanical parameters like stresses, strains or strain rates within the tissue lead to local property changes.

Hesselink (1998) induced and determined tissue damage during lengthening contractions of the rats TA muscle. It was shown that following lengthening exercise, the cytoskeletal protein vimentin was re-expressed together with desmin. After a second lengthening exercise session, vimentin expression was less abundant. This indicated the remodeling of the intermediate filament network, which may improve the force transmission and thus muscle function during lengthening contractions. By simulating the experiments of Hesselink (1998) with the 3D continuum model of the TA, the distribution of mechanical parameters within the muscle during the experiments can be evaluated. Comparing simulated mechanical parameter distributions witin the tissue to changes in the expression of cytoskeletal proteins like desmin and vimentin following the contractions, can lead to the identification of mechanical parameters which are responsible for the local property changes.

The experiments of Hesselink et al. (2001) can be used to find the relation between mechanical parameters and local property changes on a smaller (cell) level. Hesselink et al. (2001) studied $\alpha$-glucosidase knockout mice, who are used as a model for the Pompe's disease. The consequence of this disease is the development of non-contractile inclusions within the muscle cell. From muscle force measurements during isometric contractions, Hesselink et al. (2001) concluded that the non-contractile material is a potential cause of the decline in muscle function. Furthermore, an increased desmin content of the affected muscle was observed, which may be considered as an adaptive response to compensate for the disturbed force transmission. By modeling a small part of the muscle tissue including the non-contractile inclusions within the cells, the distribution of mechanical quantities during contraction near the inclusions can be simulated. The hypothesis that local mechanical parameters within the tissue lead to local property changes can subsequently be tested by comparing the simulated local mechanical parameters with changes in the expression of cytoskeletal proteins like desmin and vimentin on cell level. Besides, by implementing non-contractile areas in the continuum model of the whole muscle, the effect of the inclusions on the macroscopic muscle force can be studied.

Once the relation between local mechanical quantities and changed tissue properties is proven and identified, such a relation can be implemented in 3D continuum models to study the effect of a certain mechanical loading on macroscopic muscle function. 


\section{References}

H. Abe, K. Hayashi and M. Sato. Data book on mechanical properties of living cells, tissues, and organs. Springer - New York, 1996. ISBN 4-431-70175-3.

A. Alistair and Z.A.F. Young. Right ventricular midwall surface motion and deformation using magnetic resonance tagging. American Journal of Physiology, 270:pH281, 1996.

D.G. Allen, J. Lannergren and H. Westerblad. Muscle cell function during prolonged activity: cellular mechanisms of fatigue. Experimental Physiology, 1995.

W.A. Anderson, C.R. Bridges, A.J. Chin, J.S. Andersen, M.A. Acker, R.L. Hammond, F. Dimeo, PT. Cahalan, D.R. Gale and W.E. Brown. Long-term stimulation of skeletal muscle: its potential for a theter-free biologic cardiac assist device. Pacing Clinical Electrophysiology, 11:2128-2134, 1988.

R.B. Armstrong and R.O. Phelps. Muscle fiber type composition of the rat hindlimb. American Journal of Anatomy, pages 259-272, 1984.

L. Axel and L. Dougherty. Heart wall motion: improved method of spatial modulation of magnetization for MR imaging. Radiology, 172:349-350, 1989a.

L. Axel and L. Dougherty. MR imaging of motion with spatial modulation of magnetization. Radiology, 171:841-845, 1989b.

V. Badhwar, R.K. Badhwar, J.H. Oh and R.C. Chui. Power generation from four skeletal muscle configurations. design implications for a muscle powered cardiac assist device. ASAIO Journal, 43:M651-M657, 1997.

J.A. Bernards and L.N. Bouman. Fysiologie van de mens. Bohn, Scheltema \& Holkema, Emmalaan 27, 3581 HN Utrecht (NL), 5th edition, 1988. In Dutch.

J.A. Bernards and L.N. Bouman. Fysiologie van de mens. Bohn, Scheltema \& Holkema, Emmalaan 27, 3581 HN Utrecht (NL), 6th edition, 1996. In Dutch.

D.G. Bliss and M.B. Menelaus. The results of transfer of the tibialis anterior to the heel in patients who have a myelomeningocele. Journal Bone Joint Surgery, 68:12581264, 1986. 
A.C.B. Bogaerds. Parameterizering van geometrie en vezelveld van skeletspier uit MRI-meting. Technical Report WFW-report 97.025, Eindhoven University of Technology, 1997. In dutch.

E.H.M. Bosboom, C.V.C. Bouten, C.W.J. Oomens, H. van Straaten, F.P.T. Baaijens and H.Kuipers. Quantification and localization of damage in rat muscles after controlled loading; a new approach to study the aetiology of pressure sores. Medical Engineering and Physics, 23:195-200, 2001a.

E.H.M. Bosboom, M.K.C. Hesselink, C.W.J. Oomens, C.V.C. Bouten, M.R. Drost and F.PT. Baaijens. Passive transverse mechanical properties of skeletal muscle. Journal of Biomechanics, 34:1365-1368, 2001b.

P.H.M. Bovendeerd, J.M. Huyghe, T. Arts, D.H. van Campen and R.S. Reneman. Influence of endocardial-epicardial crossover of muscle fibers in left ventricular wall mechanics. Journal of Biomechanics, 27:941-951, 1994.

R. Brunner. Changes on muscle power following tendon lengthening and tendon transfer. Orthopade, 24:246-251, 1995.

R.C. Chiu and I.M. Bourgeois. Transformed Muscle for Cardiac Assist and Repair. Futura Publishing Company - New York, 1990. ISBN 0-87993-355-0.

J. de Ruiter. Physiological properties of skeletal muscle units vary with the intramuscular location of their fibres. Ph.D. thesis, Vrije Universiteit te Amsterdam, 1996.

S.L. Delp, D.A. Ringwelski and N.C. Carroll. Transfer of the rectus femoris: effects of transfer site on moment arms about the knee and hip. Journal of Biomechanics, 27:1201-1211, 1994.

C.B. Ebbeling and P.M. Clarkson. Exercise-induced muscle damage and adaptation. Sports Medicine, pages 207-234, 1989.

K.A.P. Edman and A.R. Mattiazzi. Effects of fatigue and altered ph on isometric force and velocity of shortening at zero load in frog muscle fibres. Journal of Muscle Research and Cell Motility, 2:321-334, 1981.

S.E. Fischer, G.C. McKinnin, M.B. Scheidegger, W. Prins, D. Meier and P. Boesiger. True myocardial motion tracking. Magnetic Resonance Medicine, 31:401-413, 1994.

R.H. Fitts, K.S. McDonald and J.M. Schluter. The determinants of skeletal muscle force and power: their adaptability with changes in activity pattern. Journal of Biomechanics, 24:111-122, 1991.

Y.C. Fung. Biomechanics, mechanical properties of living tissues. $2^{\text {nd }}$ edition. SpringerVerlag, 1993.

W.F. Ganong. review of Medical Physiology. LANGE Medical Publications, Los Altos, California 94022, 10th edition, 1981. 
K.K. Gealow, E.E. Solien, R.W. Bianco, R.C. Chui and S.J. Shumway. Conformational adaptation of muscle: implications in cardiomyoplasty and skeletal muscle ventricles. Annals of Thoraccic Surgery, 56:520-526, 1993.

M.G.D. Geers, R. de Borst and W.A.M. Brekelmans. Computing strain fields from discrete displacement fields in 2d-solids. International Journal of Solids Structures, 33:4293-4307, 1996.

A.W.J. Gielen. A continuum approach to the mechanics of contracting skeletal muscle. Ph.D. thesis, Eindhoven University of Technology, 1998.

A.W.J. Gielen, C.W.J. Oomens, P.H.M. Bovendeerd, T. Arts and J.D. Janssen. A finite element approach for skeletal muscle using a distributed moment model of contraction. Computer Methods in Biomechanics and Biomedical Engineering, 3:231244, 2000.

M. Gorselink, M.R. Drost, J. de Louw, P.J.B. Willems, N. Rosielle and N. Janssen. Accurate assessment of in situ isometric contractile properties of hind limb plantar and dorsal flexor muscle complex of intact mice. European Journal of Physiology, 439:665-670, 2000.

A.C. Guyton and J.E. Hall. London : Saunders, 2000, 10th edition, 2000.

H. Hatze. Myocybernetic control models of skeletal muscle: Characteristics and applications. University of South Africa, Muckleneuk, Pretoria, 1981.

D. Hawkins and M. Bey. Muscle and tendon force-length properties and their interactions in vivo. Journal of Biomechanics, 30:63-70, 1997.

K. Hendrich. Surface coil cardiac tagging and ${ }^{31} \mathrm{P}$ spectroscopic localization with $\mathrm{B}_{1}$-sensitive adiabatic pulses. Magnetic Resonance in Medicine, 31:541-545, 1994.

W. Herzog, S. Kamal and H.D. Clarke. Myofilament lengths of cat skeletal muscle: theoretical considerations and functional implications. Journal of Biomechanics, 25:945-948, 1992.

M.K.C. Hesselink. Structural, functional and metabolic aspects of shortening and lengthening muscle contractions. Ph.D. thesis, University Maastricht, 1998.

M.K.C. Hesselink, H. Kuipers, P. Geurten and H. van Straaten. Structural muscle damage and muscle strength after incremental number of isometric and forced lengthening contractions. Journal of Muscle Research and Cell Motility, 17:1-7, 1996.

R.P. Hesselink, M. Gorselink, G. Schaart, A.J.M. Wagemakers, J. Kamphoven, A.J.J. Reuser, G.J. van der Vusse and M.R. Drost. Impaired performance of skeletal muscle in $\alpha$-glucosidase knockout mice, 2001. To be published.

A.V. Hill. The heat of shortening and the dynamic constrants in mucle. Proceedings of the Royal Society of London, 126:136-165, 1938. 
A.V. Hill. The series elastic component of muscle. Proceedings of the Royal Society of London, 137:273-280, 1950.

PA. Huijing. Important experimental factors for skeletal muscle modelling: Nonlinear changes of muscle length force characteristics as a function of degree of activity. European Journal of Morphology, 34:47-54, 1996.

A.F. Huxley. Muscle structure and theories of contraction. Progress in Biophysics and Biophysical Chemistry, 7:257-318, 1957.

A.F. Huxley. Muscular contraction. Journal of Physiology, 243:1-43, 1974.

A.F. Huxley and R. Niedergerke. Structural changes in muscle during contraction. Nature, 173:971-973, 1954.

A.F. Huxley and L.C. Peachey. The maximum length for contraction in vertebrate straited muscle. Journal of Physiology, 156:150-165, 1961.

A.F. Huxley and R.M. Simmons. Proposed mechanism of force generation in striated muscle. Nature, 233:533-538, 1971.

A.F. Huxley and R.M. Simmons. Mechanical transients and the origin of muscle force. Cold Spring Harbor Symposia on Quantitative Biology, 37:669-680, 1973.

H. Huxley and J. Hanson. Changes in the cross-striations of muscle during contraction and stretch and their structrual interpretation. Nature, 173:973-976, 1954.

M. Illert, M. Trauner, E. Weller and E. Wiedemann. Forearm muscles of man can reverse their function after tendon transfers: an electromyographic study. Neuroscience Letters, 67:129-134, 1986.

M. Irving, T.St. Claire Allen, C. Sabido-David, J.S. Craik, B. Brandmeier, J. KendrickJones, J.E.T. Corrie, D.R. Trentham and Y.E. Goldman. Tilting of the light-chain region of myosin during step length changes and active force generation in skeletal muscle. Nature, 375:688-691, 1995.

A. Ishijima, T. Doi, K. Sakurada and T. Yanagida. Sub-piconewtion force fluctuations of actomyosin in vitro. Nature, 352:301-306, 1991.

B. Jahne. Spatio-temporal image processing, Theory and scientific applications. Springer-Verlag, 1993. Berlin, Germany.

P.G.M. De Jong, T Arts, A.P.G. Hoeks and R.S. Reneman. Determination of tissue motion velocity by correlation interpolation of pulsed ultrasonic echo signals. Ultrasonic Im, 12:84-98, 1990.

K.R. Kaufman, K.N. An and E.Y.S. Chao. Incorporation of muscle architecture into the muscle length-tension relationship. Journal of Biomechanics, 22:943-948, 1989.

M. Kawai and D. Kuntz. Optical diffraction studies of muscle fibrs. Biophysical Journal, 13:87-87, 1973. 
M.W. Keith, K.L. Kilgore, P.H. Peckham, K.S. Wuolle, G. Creasey and M. Lemay. Tendon transfers and functional electrical stimulation for restoration of hand function in spinal cord injury. Hand Surgery, 21:89-99, 1996.

T.C.S. Keller, III. Molecular bungees. Nature, 387:233-235, 1997.

R.C.P. Kerckhoffs. In vivo determination of a $2 D$ strain field in skeletal muscle using $M R$ tagging. Master's thesis, Eindhoven University of Technology, 1998. WFW-report 98.023.

T.J. Koh and W. Herzog. Excursion is important in regulating sarcomere number in the growing rabbit tibialis anterior. Journal of Physiology, 508:267-280, 1998a.

T.J. Koh and W. Herzog. Increasing the moment arm of the tibialis anterior induces structural and functional adpatation. Journal of Biomechanics, 31:593-599, 1998b.

J.P.A. Kuijer, J.T. Marcus, M.J. Götte, A.C. van Rossum and R.M. Heethaar. Threedimensional myocardial strain analysis based on short- and long-axis magnetic resonance tagged images using a 1D displacement field. Journal of Magnetic Resonance Imaging, 18:553-564, 2000.

H. Kuipers. Exercise-induced muscle damage. International Journal of Sports Medicine, 15:132-135, 1994.

S.L. Lehman. A detailed biophysical model of human extraocular muscle. Ph.D. thesis, University of California, 1982.

R.L. Lieber. Mechanical factors underlying muscle damage. In Leendert Blankevoort and Jan G. M. Kooloos, editors, Proceedings of the Second World Congress of Biomechanics, July 10-15, Amsterdam, The Netherlands., pages II-121. 1994.

R.L. Lieber and P.J. Baskin. Intersarcomere dynamics of single muscle fibres during fixed-end tetani. Journal of General Physiology, 82:347-364, 1983.

R.L. Lieber and J. Fridén. Muscle damage is not a function of muscle force but active muscle strain. Journal of Applied Physiology, 74:520-526, 1993.

R.L. Lieber, G.J. Loren and J. Fridén. In vivo measurement of human wrist extensor muscle sarcomere length changes. Journal of Neurophysiology, 71:874-881, 1994.

V. Lombardi, G. Piazzesi, M.A. Ferenczi, H. Thirwell, I. Dobbie and M. Irving. Elastic distortion of myosin heads and repriming of the working stroke in mucle. Nature, 374:553-555, 1995.

S. Ma and G.I. Zahalak. A simple self-consistent distribution-moment model for muscle: chemical energy and heat rates. Mathematical Biosciences, 84:211-230, 1987.

S. Ma and G.I. Zahalak. A distibution-moment model of energetics in skeletal muscle. Journal of Biomechanics, Vol. 24:21-35, 1991. 
S.P. Ma. Activation dynamics for a distribution-moment model of muscle.. Ph.D. thesis, Washington University, 1988.

M. Maenhout, M.K.C. Hesselink, E. Blezer, A. van der Toorn, K. Nicolay, C.W.J. Oomens and M.R. Drost. Three-dimensional strain fields of isometrically contracting rat tibialis anterior muscle based on $\mathrm{mr}$ tagged images, 2001a. Submitted to: Magnetic Resonance in Medicine.

M. Maenhout, M.K.C. Hesselink, C.W.J. Oomens and M.R. Drost. Parameter identification of a distribution-moment approximated two-state huxley model of the rat tibialis anterior muscle. In Walter Herzog, editor, Skeletal Muscle Mechanics; From Mechanism to Function, chapter 9, pages 135-154. John Wiley \& Sons, 2000. ISBN 0-471-49238-8.

M. Maenhout, M.K.C. Hesselink, C.W.J. Oomens and M.R. Drost. Effect of muscle force and ankle angle on the superficial fiber stretch ratio distribution of isometrically contracting rat tibialis anterior muscle, 2001b. To be submitted to: Journal of Biomechanics.

E.R. McVeigh and E. Atalar. Cardiac tagging with breath-hold cine MRI. Magnetic Resonance in Medicine, 28:318-327, 1992.

M.H.H. Meuwissen. An Inverse Method for the mechanical characterisation of metals. Ph.D. thesis, Eindhoven University of Technology, 1998.

H. Mizahara, T. Koshiji, K. Nishimura, S. Nomoto, Matsuda and T.Ban. Evaluation of a compressive-type skeletal muscle pump for cardiac assistance. Annals of Thoracic Surgery, 67:105-111, 1999.

J.E. Molloy, J.E. Burns, J. Kendrick-Jones, R.T. Tregear and D.S.C. White. Movement and force produced by a single myosin head. Nature, 378:209-212, 1995.

B. Moran, M. Ortiz and C.F. Shih. Formulation of implicit finite element mehtods for multiplicative finite deformation plasicity. Int. J. for Numerical Methods in Engineering, 29:483-514, 1990.

I. MotabarZadeh. A distribution-moment model of mechanical instability in nonuniform skeletal muscle fibres. Ph.D. thesis, Washington University, Saint Louis, Missouri, 1998.

A.M.M. Muijtjens, J.M.A. Roos, T. Arts and A. Hasman. 3-d reconstruction of marker positions from stereo images using ml estimation. Proceedings Conference on Medical and Biological Engineering, Jeruzalem, 1995a.

A.M.M. Muijtjens, J.M.A. Roos, T. Arts, A. Hasman and R.S. Reneman. Simultaneous estimation of stereo correspondence and camera geometry from marker tracks. Proceedings IEEE Conference of Computations in Cardiology, Wenen, pages 577-580, 1995b. IEEE Computer Society Press, Washington. 
A.M.M. Muijtjens, J.M.A. Roos, T. Arts, A. Hasman and R.S. Reneman. Tracking markers with missing data dy lower rank approcimation. Journal of Biomechanics, 30:95-98, 1997.

B.S. Myers, C.T. Woolley, T.L. Slotter, W.E. Garrett and T.M. Best. The influence of strain rate on the passive and stimulated engineering stress-large strain behavior of the rabbit tibialis anterior muscle. Journal of Biomechanical Engineering, pages 126-132, 1998.

C.W.J. Oomens, M.R.v Ratingen, J.D. Janssen, J.J. Kok and M.A.N. Hendriks. A numerical-experimental method for a mechanical characterization of biological materials. Journal of biomechanics, ISSN 0021-9290, 26:617-622, 1993.

L. Ossevoort. Measuring deformation in skeletal muscle using NMR imaging techniques. Master's thesis, Eindhoven University of Technology, 1997. WFW-report 97.034.

G. Piazzesi, M. Linari, M. Reconditi, F. Vanzi and V. Lombardi. Cross-bridge detachment and attachment following a step stretch imposed on active single frog muscle fibres. Journal of Physiology, 498:3-15, 1997.

K.M. Quapp and J.A. Weiss. Material characterization of human medial collateral ligaments. ASME, Journal of Biomechanical Engineering, 120, 1999.

J. Rijcken, P.H.M. Bovendeerd, A.J.G. Schoofs, D.H.van Campen and T. Arts. Research articles - optimization of cardiac fiber orientation for homogeneous fiber strain during ejection. Annals of biomedical engineering: the journal of the Biomedical Engineering Society, 27:289-297, 1999. ISSN 0090-6964.

J. Rijcken, P.H.M. Bovendeerd, A.J.G. Schoofs and D.H. van; T. Arts. Optimazation of cardiac fiber orientation for homogeneous fiber strain at beginning of ejection. Journal of biomechanics, 30:1041-1050, 1997.

N. Sasaki and S. Odajima. Elongation mechanism of collagen fibrils and force-strain relations of tendon at each level of structural hierarchy. Journal of Biomechanics, 29:1131-1136, 1996.

S.H. Scott and G.E. Loeb. Mechanical properties of aponeurosis and tendon of the cat soleus muscle during whole muscle isomatric contractions. Journal of Morphlogy, 224:73-86, 1995.

L. Skubiszak. Mechanism of muscle contraction. Technology and Health Care, 1:133142, 1993.

W.T. Stauber. Eccentric action of muscles: Physiology, injury and adaptation. Exercise and Sportsscience review, pages 157-185, 1989.

S.D. Thomas, Jr. and E.R. McVeigh. Model-free reconstruction of three-dimensional myocardial strain from planar tagged MR images. Journal of Magnetic Resonance Imaging, 7:799-881, 1997. 
J.G. Tidball. Force transmission across muscle cell membranes. Journal of Biomechanics, 24 supp 1:43-52, 1991.

A. Tobie. Sarcomere lenght measurements using a laser diffraction technique. Master's thesis, Eindhoven University of Technology and ICAM, 1997. WFW-report 97.037.

C.L. Trestik and R.L. Lieber. Relationschip between achilles tendon mechanical properties and gastrocnemius muscle function. Journal of Biomechanical Engineering, 115:225-230, 1993.

D.R. Trumble, W.A. LaFramboise, C. Duan and J.A. Magovern. Functional properties of conditioned skeletal muscle: implications for muscle-powered cardiac assist. American Journal of Physiology, 273:C588-C597, 1997.

L. Tskhovrebova, J. Trinick, J.A. Sleep and R.M. Simmons. Elasticity and unfolding of single molecules of the giant muscle protein titin. Nature, 387:308-312, 1997.

H. van Bavel, M.R. Drost, J.D.L. Wielders, J.M. Huyghe, A. Huson and J.D. Janssen. Strain distribution on rat medial gastrocnemius (MG) during passive stretch. Journal of Biomechanics, 29:1069-1074, 1996.

B.J.J.J. van der Linden, H.F.J.M. Koopman, P.A. Huijing and H.J. Grootenboer. A finit element skeletal muscle model for simulation of isometric and concentric contractions. Comp Meth Biomech Biomed Eng, 1998a. In press.

B.J.J.J. van der Linden, H.F.J.M. Koopman, P.A. Huijing and H.J. Grootenboer. Revised planimetric model of unipennate skeletal muscle: a mechanical approach. Clinical Biomechanics, 13:256-260, 1998b.

J.H. van der Meulen. Execise-induced muscle damage: morphological, biochemical and functional aspects. Ph.D. thesis, Rijksuniversiteit Limburg (NL), 1991.

J.H. van der Meulen, H. Kuipers, J.C. van der Wal and J. Drukker. Quatitative and spatial aspects of degenerative changes in rat soleus muscle after exercise of different durations. Journal of Anatomy, pages 349-353, 1993.

C.C. van Donkelaar, L.J.G. Kretzers, P.H.M. Bovendeerd, L.M.A. Lataster, K. Nicolay, J.D. Janssen and M.R. Drost. Diffusion tensor imaging in biomechanical studies of skeletal muscle function. Journal of Anatomy, 194:79-88, 1999a.

C.C. van Donkelaar, P.J.B. Willems, A.M.M. Muytjens and M.R. Drost. Skeletal muscle transverse strain during isometric contraction at different lengths. Journal of Biomechanics, 32:755-762, 1999b.

A. van Doorn, P.H.M. Bovendeerd, K. Nicolay, M.R. Drost and J.D. Janssen. Determination of muscle fibre orientation using Diffusion-Weighted MRI. European Journal of Morphology, 34:5-10, 1996.

J.L. van Leeuwen and C.W. Spoor. Modelling mechanically stable muscle architectures. Phil. Trans. R. Soc. Lond. B, 336:275-292, 1992. 
J.L. van Leeuwen and C.W. Spoor. A two dimensional model for the prediction of muscle shape and intramuscular pressure. European Journal of Morphology, 34:2530, 1996.

C. van Oijen, F.N. van de Vosse and F.PT. Baaijens. An updated lagrange formulation of a constitutive model for incompressible fiber-reinforced materials at finite strains. 2001. To be submitted.

W.J. Vankan, J.M. Huyghe, D.W. Slaaf, C.C. van Donkelaar, M.R. Drost, J.D. Janssen and A. Huson. Finit element simulation of blood perfusion in muscle tissue during compression and sustained contraction. American Journal of Physiology, 273:H1587-H1594, 1997.

W.J. Vankan, J.M. Huyghe, C.C. van Donkelaar, M.R. Drost, J.D. Janssen and A. Huson. Mechanical blood-tissue interacting muscle: a model study. Journal of Biomechanics, 31:401-409, 1998.

P.A. Watson. Function follows form: generation of intracellular signals by cell deformation. FASEB J., 5:2013-2019, 1991.

H. Westerblad and J. Lännergren. Slowing of relaxation during fatigue in single mouse muscle fibres. Journal of Physioloy, 434:323-336, 1991.

J.M. Winters and S.L-Y Woo. Multiple muscle systems: biomechanics and movement organization. Springer-Verlag, New York, 1990.

R.D. Woittiez, P.A. Huijing, H.B.K. Boom and R.H. Rozendal. A three-dimensional muscle model: a quantified relation between form and function of skeletal muscle. Journal of Morphology, 182:95-113, 1984.

E. Yamamoto, K. Hayashi and N. Yamamoto. Mechanical properties of collagen fascicles from the rabbit pattelar tendon. ASME, Journal of Biomechanical Engineering, 121, 1999.

G.I. Zahalak. A distribution-moment approximation for kinetic theories of muscular contraction. Mathematical Biosciences, 55:89-114, 1981.

G.I. Zahalak. A comparison of the mechanical behavior of the cat soleus muscle with a distribution-moment model. Journal of Biomechanical Engineering, 108:131-140, 1986.

G.I. Zahalak. Non-axial muscle stress and stiffness. Journal of Theoretical Biology, 182:59-84, 1996.

G.I. Zahalak. Can muscle fibers be stable on the descending limbs of their sarcomere length-tension relations? Journal of Biomechanics, 30:1179-1182, 1997.

G.I. Zahalak. Rule of thumb for the relation between $f_{1}$ and $g_{1}$. Personal Communications, 1999. 
G.I. Zahalak and S. Ma. Muscle activation and contraction: constitutive relations based directly on cross-bridge kinetics. Journal of Biomechanical Engineering, 112:52-62, 1990.

G.I. Zahalak and I. Motabarzadeh. A re-examination of calcium activation in the huxley cross-bridge model. Journal of Biomechanical Engineering, 119:20-29, 1997.

C.J. Zuurbier and P.A. Huijing. Changes in geometry of actively shortening unipennate rat gastrocnemius muscle. Journal of Morphology, pages 167-180, 1993. 


\section{Samenvatting}

Mechanische belasting van spierweefsel leidt tot inhomoge spannings- en rekverdelingen binnen het weefsel en eventueel tot lokale schade en adaptatie. De spannings- en rekverdelingen resulteren in lokale belasting, waarvan wordt aangenomen dat deze verantwoordelijk is voor lokaal veranderende eigenschappen. Kennis omtrent de mechanismen van het ontstaan van lokale schade en adaptatie als gevolg van mechanische belasting is belangrijk voor verschillende toepassingsgebieden, waaronder 'myoplasty' en pees- of spierverleggingen. Een voorbeeld van myoplasty is de ondersteuning van de pompfunctie van het hart door middel van een getransformeerde skeletspier. Pees- en spierverleggingen worden gebruikt voor het herstellen van de mobiliteit van aangetaste gewrichten door ruggemerg beschadigingen of om een afname in kniebewegingen tijdens lopen te herstellen. Ook de ontwikkeling van drukwonden is een klinisch toepassingsgebied. Het is bekend dat spierweefsel gevoelig is voor langdurige transversale belasting en drukwonden beginnen vaak in de dieper gelegen spierlagen in de buurt van bot uitsteeksels. Algemeen wordt aangenomen dat schade en adaptatie op een lokaal (cel) niveau ontstaan.

Continuüm modellen van het actieve en passieve mechanische gedrag van spieren met realistische geometrieën in in vivo situaties vormen een zeer bruikbaar middel om lokale mechanische belasting van spieren in relatie tot lokale schade en adaptatie te bestuderen. Met dit doel heeft Gielen et al. (2000) een continuüm model ontwikkeld van het mid-sagittaal vlak van de tibialis anterior (TA) van de rat.

Het doel van het onderzoek beschreven in dit proefschrift was 1 ) het verzamelen van experimentele data om continuüm modellen van de TA van de rat te valideren en 2) het verbeteren van het 2-dimensionaal (2D) model van Gielen et al. (2000), vooral met betrekking tot de geometrie. Met het sagittale vlak model kunnen verschillende output parameters geëvalueerd worden (bijv. spierkracht, verplaatsingen, vezelrek, rekgradiënten). Er is daarom voor gekozen om met verschillende meettechnieken een uitgebreide experimentele data set te verzamelen.

De eerste experimenten, beschreven in hoofdstuk 3 , waren gericht op het identificeren 
van parameters van het gebruikte contractie model, het zogenaamde 'two-state Huxley' model. De identificatie omvatte het fitten van een 1-dimensionaal (1D) model van een spier-pees complex op experimenteel verkregen spierkoppels tijdens isometrische contractie bij verschillende stimulatie frequenties. De resultaten lieten zien dat de geïdentificeerde model parameters een goede beschrijving van spierkoppels van de TA van de rat bij verschillende stimulatie frequenties mogelijk maken. Er wordt daarom aangenomen dat het 'two-state Huxley' contractie model toereikend is voor het beschrijven van het contractiel gedrag binnen continuüm modellen van complete spieren tijdens isometrische contracties. Verder maakt het contractie model toekomstige studies naar metabole processen mogelijk. De experimenteel waargenomen koppel relaxatie snelheid werd echter overschat door het 1D model. Dit kan veroorzaakt zijn door ruimtelijke effecten, die in de toekomst bestudeerd kunnen worden met continuüm modellen.

Met 3-dimensionale (3D) video analyse zijn oppervlakkige marker verplaatsingen gemeten tijdens een isometrisch contraherende TA spier van een rat door electrostimulatie (hoofdstuk 4). Er is geëvalueerd of de gemeten rekverdelingen afhangen van 1) de spierkracht, 2) de gewrichtshoek van de enkel. Daarnaast is bekeken hoe de rekverdeling veranderde tijdens opbouw en relaxatie van de spierkracht. De berekende rekken tijdens de plateau fase waren sterk afhankelijk van de longitudinale positie. De resultaten lieten verder zien dat een krachtsvariatie van minder dan $20 \%$ geen significante invloed heeft op lokale vervorming. Daarnaast bleek een verschil in de enkel hoek van ongeveer $30^{\circ}$ een verschil in rek te veroorzaken van 0.01 (onafhankelijk van de longitudinale positie). De rek-range in logitudinale richting was 0.17 . Hieruit is geconcludeerd dat kleine verschillen in de enkelhoek (orde $5^{\circ}$ ) naar verwachting geen significante verschillen in de lokale deformatie veroorzaken. Deze bevindingen kunnen belangrijk zijn bij het ontwerpen van schade inducerende inspanningsprotocollen, die gebruikt kunnen worden om de relatie te vinden tussen lokale schade en lokale mechanische parameters. Een afname in de spierkracht van $20 \%$ door vermoeidheid kan worden geaccepteerd en kleine verschillen in de enkel hoek zullen geen grote invloed hebben op de rekverdelingen. Naast de relevantie voor het ontwikkelen van inspanningsprotocollen, kan de experimenteel bepaalde relatie tussen lokale rekken en respectievelijk spierkracht en spierlengte dienen ter validatie van continuüm modellen. Tijdens de krachtsopbouw en relaxatie werden onverwachte effecten waargenomen. Aan het begin van de stimulatie verkortte het proximale weefsel sneller dan het distale weefsel. Voordat de initiele toestand werd bereikt, trad tijdens de krachtsrelaxatie een extra verkorting op in het distale weefsel, terwijl de proximale delen verlengden. De gemeten effecten hebben tot de hypothese geleid dat de voortgeleidingssnelheid van de actie potentiaal vanuit de motorische eindplaatjes over de spiervezels het tijdsverloop van de lokale rekverdelingen sterk 
beïnvloedt. De hypothese kan bestudeerd worden door inhomogene activatie van de spiervezels te implementeren in de continuüm modellen.

Om de interne 3D vervorming tijdens de plateau fase van een gefuseerde isometrische contractie te bepalen, is gebruik gemaakt van MRI-tagging (hoofdstuk 5). Uit twee sets van orhogonale MR beelden met weefsel tags van een isometrisch samentrekkende TA spier van de rat zijn 3D verplaatsings- en rekvelden bepaald van ongeveer $70 \%$ van het spiervolume. Om de bruikbaarheid van oppervlakkige rekken als maat voor diepere rekken te testen, zijn uit de verplaatsingsvelden sagitale rekgradiënten bepaald. Daarnaast zijn ook de transversale rekgradiënten bepaald, om te achterhalen of sagitale vlak modellen bruikbaar zijn voor het beschrijven van de verdeling van mechanische grootheden binnen de spier. Het grootste verschil in rek in alle richtingen over een afstand van $1 \mathrm{~mm}$ in sagitale en transversale richting was $50 \%$ van de maximale absolute rek. De rekverdelingen binnen de contraherende spier zijn dus sterk heterogeen. De conclusie was daarom 1) dat oppervlakkige rekken geen goede maat zijn voor diepere rekken en 2) dat er modellen met realistische geometrieën nodig zijn voor het bestuderen van 3D spiermechanica tijdens contractie. De uitgebreiding van het 2D model van het mid-sagitale vlak van de tibialis anterior van de rat (Gielen et al., 2000) naar een meer realistische 3D geometrie is beschreven in hoofdstuk 6. De behoudswetten zijn opgelost met de eindige elementen methode door gebruik te maken van een gemengde, 'updated Lagrange' formulering. Verder is er gebruik gemaakt van een nieuw vaste stof element, waardoor de robuustheid van de berekeningen van incompressibel gedrag is verbeterd. Het verschil tussen simulaties van rek en verplaatsingsvelden met een 2D en 3D geometrie is geëvalueerd, evenals het verschil tussen simulaties met verschillende sarcomeerlengte verdelingen (zie tabel 6.3, hoofdstuk 6). Ook is er een stap gezet in de richting van modelvalidatie. Het verschil tussen de $2 \mathrm{D}$ en $3 \mathrm{D}$ simulaties was uitsluitend prominent voor de verplaatingen in transversale richting. De initiële sarcomeer lengte verdeling had vooral invloed op de verplaatsingen en rekken in de longitudinale richting.

Met betrekking tot de modelvalidatie is het duidelijk geworden dat de simulaties een goede kwalitatieve voorspelling van de MRI verplaatsingsvelden mogelijk maakten. Er werden echter grote kwalitatieve verschillen gevonden. Voor het nauwkeurig beschrijven van de gemeten verplaatsings- en rekvelden zal het model verbeterd moeten worden. In toekomstige studies zou de focus moeten liggen op de volgende parameters: de 3D geometrie en vezel richting, de randvoorwaarden, de constitutieve wetten voor passief gedrag, en de lokale sarcomeerlengte verdelingen. In paragraaf 6.4 .2 worden deze parameters uitvoerig besproken. Ondanks de benodigde model verbeteringen, biedt het gepresenteerde 3D model samen met de uitgebreide experimentele data set, een solide gereedschap om de spiermechanica tijdens contractie te bestuderen. 
110 Samenvatting 


\section{Dankwoord}

Het tot stand brengen van dit proefschrift was me zonder de hulp van veel mensen zeker niet gelukt. Op deze plaats wil ik dan ook mijn dank doen blijken aan al diegenen die, op wat voor manier dan ook, een steentje hebben bijgedragen. Een aantal mensen wil ik in het bijzonder bedanken.

Om te beginnen verdienen mijn promotoren Frank Baaijens, Harm Kuipers en oudpromotor Jan Janssen, mijn dank voor de begeleiding en het mogelijk maken van het onderzoek.

Van mijn co-promotoren Cees Oomens en Maarten Drost heb ik veel geleerd. Als begeleidingsteam vulden jullie elkaar perfect aan, dus wat mij betreft kan een AIO zich geen beter co-promotoren duo wensen. Veel dank daarvoor.

Paula Steffens heeft middels haar afstudeerwerk en stage een bijdrage gelevert aan het onderzoek. Verder waren ook Leonie van de Heuvel, Henry van de Zilver en Wouter Wilson als stagiair betrokken bij het onderzoek. Op deze plaats wil ik jullie allen hartelijk danken voor jullie bijdragen.

Het ontwikkelen, plannen en uitvoeren van de experimenten heb ik als een van de leukste onderdelen van mijn promotie ervaren. Vanwege het multidiciplinaire karakter van de experimenten had ik de hulp van een aantal specialisten hard nodig en hen ben ik daarom zeer dankbaar. Tijdens de experimenten bleef de sfeer, ondanks hier en daar een tegenslag, altijd goed. Ik zal hier in de toekomst met veel plezier aan terug denken. Vooral Maarten Drost verdient voor dit alles mijn speciale dank, maar zeker ook Mattijs Hesselink, Erwin Blezer, Paul Willems, Marijn Kruiskamp, Klaas Nicolay, Robert van Sluis, Gerard van Vliet, Annette van der Toorn, Rob van de Berg en Erwin Dekkers.

Het programmeren van eindige elementen code bleek al snel een minder favoriet onderdeel van mijn werk te zijn. Cees Oomens heeft veel van dat onderdeel op zich genomen en gelukkig deed hij dat wel met veel plezier. Cees, mijn speciale dank hiervoor. Zonder jouw inspanningen was dit boekje niet zo'n mooi geheel geworden. Mijn tijd als AIO werd ook zeker de moeite waard gemaakt door veel leuke collega's, waaronder mijn mede AIO's en vooral mijn kamergenoten. Dank jullie allen voor het 


\section{Dankwoord}

mogelijk maken van het delen van lief en soms ook leed.

Mijn ouders, Lea en Henk Vlijm, hebben op de achtergrond ook zeker een bijdrage geleverd en daarvoor wil ik ze heel erg bedanken. Mam, onze wekelijkse telefoongesprekken over soms niks, maar vaak ook over de diepere dingen van het leven geven mij meer steun en moed dan je misschien beseft. Je bent een schat! En dan natuurlijk mijn lieve Chris. Mijn bewondering voor jouw geduld en relativeringsvermogen is groot en ik kan daar nog veel van leren. Ik ben je vreselijk dankbaar voor al je aanmoedigingen en dat je er altijd voor me bent.

Tot slot lieve pap, is dit boekje voor jou. Omdat we veel te veel van elkaar moeten missen.

Mascha Maenhout

Eindhoven, januari 2002 


\section{Curriculum Vitae}

Mascha Maenhout werd geboren op 27 maart 1972 in Terneuzen. In 1990 behaalde zij haar VWO diploma aan het Zeldenrust College te Terneuzen. Aansluitend studeerde zij aan de Technische Universiteit Eindhoven. Nadat zij haar propedeuse Werktuigbouwkunde behaald had, volgde zij de pospropedeutische opleiding Werktuigkundige Medische Technologie. In augustus 1996 studeerde zij af bij het Philips Natuurkundig Laboratorium bij de afdeling Personal Care Institute. Tijdens haar afstudeerproject heeft zij gewerkt aan de karakterisatie van het mechanische gedrag van menselijk haar in relatie tot temperatuur en vochtigheid ter verbetering van 'hair care' produkten. Vanwege haar interesse in biomechanische onderzoek begon zij in 1997 als assistent in opleiding aan haar promotie-onderzoek, waarvan dit proefschrift het resultaat is. Het onderzoek viel binnen het samenwerkingsverband van de Technische Universiteit Eindhoven en de Universiteit Maastricht. 


\section{Strain fields within contracting skeletal muscle}

Local loads within muscle tissue are believed to initiate local muscle damage and adaptation. To improve the understanding of the mechanism of local damage and adaptation, knowledge is required on how external loads are transferred to local loads within the tissue. In the present thesis a continuum model of the rat TA muscle is employed and improved to investigate this transfer of loads during muscle contraction. An extensive experimental data set was acquired for validation purposes. This data set includes muscle torque measurements and strain field measurements during contraction using video analyses of surface markers and MRI tagging. The model combined with the experimental data set offers a solid tool to improve the insights in muscle mechanics, damage, and adaptation. 